\title{
Search for gauge extensions of the MSSM at the LHC
}

\author{
Ahmed Ali, ${ }^{1, *}$ Durmuş A. Demir, ${ }^{1,2, \dagger}$ Mariana Frank, ${ }^{3, \ddagger}$ and Ismail Turan ${ }^{3, \S}$ \\ ${ }^{1}$ Deutsches Elektronen - Synchrotron DESY, Notkestr. 85, D-22603 Hamburg, Germany \\ ${ }^{2}$ Department of Physics, Izmir Institute of Technology, IZTECH, TR35430 Izmir, Turkey \\ ${ }^{3}$ Department of Physics, Concordia University, 7141 Sherbrooke St. West, Montreal, Quebec, Canada H4B 1R6
}

(Received 26 February 2009; published 6 May 2009)

\begin{abstract}
The extensions of the minimal supersymmetric model (MSSM), driving mainly from the need to solve the $\mu$ problem, involve novel matter species and gauge groups. These extended MSSM models can be searched for at the LHC via the effects of the gauge and Higgs bosons or their fermionic partners. Traditionally, the focus has been on the study of the extra forces induced by the new gauge and Higgs bosons present in such models. An alternative way of studying such effects is through the superpartners of matter species and the gauge forces. We thus consider a $U(1)^{\prime}$ gauge extension of the MSSM, and perform an extensive study of the signatures of the model through the production and decays of the scalar quarks and gluino, which are expected to be produced copiously at the LHC. After a detailed study of the distinctive features of such models with regard to the signatures at the LHC, we carry out a detailed Monte Carlo analysis of the signals from the process $p p \rightarrow n$ leptons $+m$ jets $+\mathbb{E}_{T}$, and compare the resulting distributions with those predicted by the MSSM. Our results show that the searches for the extra gauge interactions in the supersymmetric framework can proceed not only through the forces mediated by the gauge and Higgs bosons but also through the superpartner forces mediated by the gauge and Higgs fermions. Analysis of the events induced by the squark/gluino decays presented here is complementary to the direct $Z^{\prime}$ searches at the LHC.
\end{abstract}

DOI: 10.1103/PhysRevD.79.095001

PACS numbers: 12.60.Cn, 12.60.Jv, 14.80.Ly

\section{INTRODUCTION AND MOTIVATION}

Any anticipated model of "new physics," which must obligatorily rehabilitate the unnatural ultraviolet sensitivity of the standard model (SM), generically involves new matter species and interactions beyond the SM. These non-SM features, if discernible in the $\mathrm{TeV}$ domain, will be probed by experiments at the LHC. The search for the non-SM gauge interactions is of particular importance since non-SM gauge forces at the weak scale can give important hints about the symmetries of nature at short distances. The search can be carried out by measuring the anomalies in the rates of scattering processes that involve solely the SM particles. For instance, $2 \rightarrow 2$ scatterings can receive contributions from the exchanges of the extra gauge bosons $Z^{\prime}$ or $W^{\prime}$, or extra Higgs bosons, and their effects can be disentangled by measuring the deviation of the scattering rate from its SM expectation. However, the effects of the non-SM gauge interactions are not limited to such processes since they necessarily participate in interactions of the non-SM particles, too. This feature extends the search procedure for extra gauge forces into non-SM particle sector, and can prove useful in establishing the inner consistency of the model of "new physics."

The search strategies for, and the signatures of, the extra gauge interactions depend crucially on the structure of the

\footnotetext{
*ahmed.ali@desy.de

†demir@physics.iztech.edu.tr

mfrank@alcor.concordia.ca

\$ituran@physics.concordia.ca
}

model of "new physics." Indeed, possible selection rules, and correlations among observables can give rise to distinctive signatures for certain scattering processes. These observations can be made explicit by considering a specific model of "new physics." To this end, TeV-scale gravity, made possible by large extra dimensions, and TeV-scale softly-broken supersymmetric theories stand up as two main avenues for constructing realistic models. Supersymmetric theories offer a viable framework for elucidating these observations, as in these theories the entire particle spectrum is paired to have the bosonfermion symmetry, and thus, quadratic divergences that destabilize the scalar field sector are naturally avoided. In particular, gauge bosons themselves are paired with the corresponding gauge fermions, and this feature guarantees that any scattering process involving the gauge bosons possesses a partner process proceeding with the gauge fermions (along with the exchange of fermions and scalar fermions). This implies that the search for extended gauge structures can be performed via both gauge bosons and gauge fermions, and the correlations between the two can reveal the underlying supersymmetric structure. The theories in higher dimensions, unless endowed with supersymmetry, do not possess this partnership structure; that is, their forces (induced by the extended gauge sector or the Kaluza-Klein modes of the known gauge fields in the bulk) do not acquire contributions from any partner.

In this paper we perform a phenomenological study of the extra gauge interactions in the context of an extended low-energy softly-broken supersymmetric model. The 
minimal supersymmetric model (MSSM) is based on the SM gauge group $G_{\mathrm{SM}}=S U(3)_{c} \otimes S U(2)_{L} \otimes U(1)_{Y}$. In general, provided that the existing bounds are respected, this gauge structure can be extended in various ways motivated by high-energy supersymmetric grand unified theories (SUSY GUTs) or strings or low-energy (the $\mu$ problem of the minimal supersymmetry, the neutrino masses or the cold dark matter) considerations. The simplest option would be to consider an extra Abelian symmetry orthogonal to $G_{\mathrm{SM}}$ so that the gauge structure at the $\mathrm{TeV}$ scale takes the form $G_{\mathrm{SM}} \otimes U(1)^{\prime}$. For extending the gauge structure there are other possibilities as well. For example, one can consider a left-right symmetric setup $S U(3)_{c} \otimes S U(2)_{L} \otimes S U(2)_{R} \otimes U(1)_{B-L}$ or a more general embedding $S U(3)_{c} \otimes S U(3)_{L} \otimes U(1)^{\prime}$. Each gauge structure comes with its associated (neutral and charged) gauge bosons and the corresponding gauginos, and their searches will help establish the underlying supersymmetric structure.

In this work we attempt to answer the following question: What are the basic collider signatures of an extended gauge structure within a supersymmetric framework? The answer involves both the forces mediated by the gauge bosons and the superpartner forces mediated by the gauge fermions. We will answer this question within the following framework:

(i) We will consider $G_{\mathrm{SM}} \otimes U(1)^{\prime}$ gauge group for definiteness (more general gauge structures can be analyzed along the lines of reasoning employed for $\left.U(1)^{\prime}\right)$.

(ii) We will analyze the production and decay processes pertaining to the LHC (processes at other colliders like Tevatron or the ILC can be analyzed accordingly).

This setup might seem too specific to investigate at first sight; however, it will be seen at the end of this analysis, that the results obtained here are sufficiently generic.

This paper is organized as follows: In Sec. II, we give a description of the features of the $G_{\mathrm{SM}} \otimes U(1)^{\prime}$ model. As several model presentations exist in the literature, we review the features essential for our analysis, relegating the rest to the Appendices for completeness. In Sec. III, we provide a general discussion of the LHC processes characteristic of the $G_{\mathrm{SM}} \otimes U(1)^{\prime}$ model. In Sec. IV, we analyze these scattering processes via Monte Carlo simulations. We summarize and conclude in Sec. V. The Lagrangian of the $G_{\mathrm{SM}} \otimes U(1)^{\prime}$ model is detailed in Appendices A, B, C, and D. For the remainder of this work, we will refer to our model simply as the $U(1)^{\prime}$ model.

\section{THE $\boldsymbol{U}(1)^{\prime}$ MODEL}

There are various reasons for extending the MSSM by an additional $U(1)$ group. From the point of view of high energies, an extra $U(1)$ symmetry broken at the $\mathrm{TeV}$ scale frequently arises in grand unified theories and strings [1].
Seen from the low-energy point of view, introduction of an extra $U(1)$ is motivated by the need to solve the $\mu$ problem [2] of the MSSM. Indeed, if the $U(1)_{Y^{\prime}}$ charges of the MSSM Higgs doublets do not sum up to zero it then becomes possible to promote the $\mu$ parameter to a SMsinglet chiral superfield $\hat{S}$ charged solely under the $U(1)_{Y^{\prime}}$ group. This setup, as encoded in the superpotential

$$
\begin{aligned}
\hat{W}= & h_{s} \hat{S} \hat{H}_{u} \cdot \hat{H}_{d}+h_{u} \hat{Q} \cdot \hat{H}_{u} \hat{U}+h_{d} \hat{Q} \cdot \hat{H}_{d} \hat{D} \\
& +h_{e} \hat{L} \cdot \hat{H}_{d} \hat{E},
\end{aligned}
$$

then induces an effective $\mu$ parameter, $\mu_{\text {eff }}=h_{s}\langle S\rangle$, below the $U(1)_{Y^{\prime}}$ breaking scale. The extra chiral field $\hat{S}$ extends (i) the MSSM Higgs sector via the additional Higgs field $S$, and (ii) the MSSM neutralino sector via the additional neutral fermion $\tilde{S}$ [3].

The other source of deviation from the MSSM stems from the presence of the extra gauge boson and its superpartner. Indeed, the kinetic terms of the gauge superfields in the electroweak sector are given by $[4,5]$

$$
\begin{aligned}
\mathcal{L}_{\text {gauge }}= & \frac{1}{32}\left[\hat{W}^{a} \hat{W}^{a}+\hat{W}_{Y} \hat{W}_{Y}+\hat{W}_{Y^{\prime}} \hat{W}_{Y^{\prime}}\right. \\
& \left.+2 \sin \chi \hat{W}_{Y} \hat{W}_{Y^{\prime}}\right]_{F},
\end{aligned}
$$

where $\hat{W}^{a}, \hat{W}_{Y}$ and $\hat{W}_{Y^{\prime}}$ are, respectively, the gauge superfields of $S U(2)_{L}, U(1)_{Y}$ and $U(1)_{Y^{\prime}}$ groups with the gauge couplings $g_{2}, g_{Y}$ and $g_{Y^{\prime}}$. The last term in (2) accounts for the kinetic mixing (with the angle $\chi$ ) between the $U(1)_{Y}$ and the $U(1)_{Y^{\prime}}$ gauge superfields. Eliminating the kinetic mixing in (2), while maintaining the hypercharge sector as in the MSSM, changes the $U(1)_{Y^{\prime}}$ invariance to a new one $U(1)_{Q^{\prime}}$ with the charge

$$
Q_{f}^{\prime}=\frac{1}{g_{Y^{\prime}} \cos \chi}\left(g_{Y^{\prime}} Y_{f}^{\prime}-g_{Y} Y_{f} \sin \chi\right),
$$

from which it follows that even if $f$ is neutral under $U(1)_{Y^{\prime}}$ it still possesses a nonvanishing charge $Q_{f}^{\prime}$ proportional to its hypercharge times $\tan \chi$. As our analysis is concerned with the superpartner fermion forces, we present that sector next. In Appendix A we describe the particle spectrum and the Lagrangian and analyze the gauge and Higgs boson sectors.

\section{Gauge and Higgs fermions}

The $U(1)^{\prime}$ model possesses no new charged Higgsinos and gauginos. On the other hand, in the neutral sector it possesses two new fermion fields: the $U(1)^{\prime}$ gauge fermion $\tilde{Z}^{\prime}$ and the singlino $\tilde{S}$. In total, there are 6 neutralino states $\tilde{\chi}_{i}^{0}(i=1, \ldots, 6)[5,6]$ :

$$
\tilde{\chi}_{i}^{0}=\sum_{a} N_{i a}^{0} \tilde{G}_{a}
$$

where the mixing matrix $N_{i a}^{0}$ connects the gauge-basis neutral fermion states $\tilde{G}_{a} \in\left\{\tilde{B}, \tilde{W}^{3}, \tilde{H}_{d}^{0}, \tilde{H}_{u}^{0}, \tilde{S}, \tilde{Z}^{\prime}\right\}$ to the 
physical neutralinos $\tilde{\chi}_{i}^{0}$. The neutralino masses $M_{\tilde{\chi}_{i}^{0}}$ and the mixing matrix $N_{i a}^{0}$ are determined via the diagonalization condition $N^{0} \mathcal{M} N^{0 T}=\operatorname{Diag}\left\{M_{\tilde{\chi}_{1}^{0}}, \ldots, M_{\tilde{\chi}_{6}^{0}}\right\}$ for the neutral fermion mass matrix

$$
\mathcal{M}=\left(\begin{array}{cccccc}
M_{\tilde{Y}} & 0 & -M_{\tilde{Y} \tilde{H}_{d}} & M_{\tilde{Y} \tilde{H}_{u}} & 0 & M_{\tilde{Y} \tilde{Z^{\prime}}} \\
0 & M_{\tilde{W}} & M_{\tilde{W} \tilde{H}_{d}} & -M_{\tilde{W} \tilde{H}_{u}} & 0 & 0 \\
-M_{\tilde{Y} \tilde{H}_{d}} & M_{\tilde{W} \tilde{H}_{d}} & 0 & -\mu & -\mu_{H_{u}} & \mu_{H_{d}}^{\prime} \\
M_{\tilde{Y} \tilde{H}_{u}} & M_{\tilde{W} \tilde{H}_{d}} & -\mu & 0 & -\mu_{H_{d}} & \mu_{H_{u}}^{\prime} \\
0 & 0 & -\mu_{H_{u}} & -\mu_{H_{d}} & 0 & \mu_{S}^{\prime} \\
M_{\tilde{Y} \tilde{Z}^{\prime}} & 0 & \mu_{H_{d}}^{\prime} & \mu_{H_{u}}^{\prime} & \mu_{S}^{\prime} & M_{\tilde{Z}^{\prime}}^{\prime}
\end{array}\right),
$$

where certain entries are generated by the soft-breaking sector while others follow from the $S U(3)_{c} \otimes S U(2)_{L} \otimes$ $U(1)_{Y} \otimes U(1)_{Q^{\prime}}$ breaking. The $U(1)_{Y}$ gaugino mass $M_{\tilde{Y}}$, the $S U(2)_{L}$ gaugino mass $M_{\tilde{W}}$, and the $U(1)_{Q^{\prime}}$ gaugino mass

$$
M_{\tilde{Z}^{\prime}}=\frac{M_{\tilde{Y}^{\prime}}}{\cos ^{2} \chi}-2 \frac{\tan \chi}{\cos \chi} M_{\tilde{Y} \tilde{Y}^{\prime}}+M_{\tilde{Y}} \tan ^{2} \chi,
$$

as well as the mixing mass parameter between $U(1)_{Y}$ and $U(1)_{Q^{\prime}}$ gauginos

$$
M_{\tilde{Y} \tilde{Z}^{\prime}}=\frac{M_{\tilde{Y} \tilde{Y}^{\prime}}}{\cos \chi}-M_{\tilde{Y}} \tan \chi,
$$

all follow from the soft-breaking sector (see Appendix A). Through the mixing of the gauge bosons, $M_{\tilde{Z}^{\prime}}$ and $M_{\tilde{Y} \tilde{Z}^{\prime}}$ exhibit an explicit dependence on the masses of the $U(1)_{Y}$ and $U(1)_{Y^{\prime}}$ gauginos, and their mass mixing. $M_{\tilde{Y} \tilde{Y}^{\prime}}$ is the soft-breaking mass that mixes the $U(1)_{Y}$ and $U(1)_{Y^{\prime}}$ gauginos.

The remaining entries in (5) are generated by the softbreaking masses in the Higgs sector via the $S U(3)_{c} \otimes$ $S U(2)_{L} \otimes U(1)_{Y} \otimes U(1)_{Q^{\prime}}$ breaking. Their explicit expressions are given by

$$
\begin{gathered}
M_{\tilde{Y} \tilde{H}_{d}}=M_{Z} \sin \theta_{W} \cos \beta, \quad M_{\tilde{Y} \tilde{H}_{u}}=M_{Z} \sin \theta_{W} \sin \beta, \\
M_{\tilde{W} \tilde{H}_{d}}=M_{Z} \cos \theta_{W} \cos \beta, \quad M_{\tilde{W} \tilde{H}_{u}}=M_{Z} \cos \theta_{W} \sin \beta, \\
\mu=h_{s} \frac{v_{s}}{\sqrt{2}}, \quad \mu_{H_{d}}=h_{s} \frac{v_{d}}{\sqrt{2}}, \quad \mu_{H_{u}}=h_{s} \frac{v_{u}}{\sqrt{2}}, \\
\mu_{H_{d}}^{\prime}=g_{Y^{\prime}} Q_{H_{d}}^{\prime} v_{d}, \quad \mu_{H_{u}}^{\prime}=g_{Y^{\prime}} Q_{H_{u}}^{\prime} v_{u}, \mu_{S}^{\prime}=g_{Y^{\prime}} Q_{S}^{\prime} v_{s},
\end{gathered}
$$

out of which only $\mu$ and $\mu_{S}^{\prime}$ involve $v_{s}$. These entries scale with $M_{Z^{\prime}}$, and thus, the heavier the $Z^{\prime}$ boson, the larger the $\tilde{S}-\tilde{Z}^{\prime}$ mixing.

The lightest neutralino $\tilde{\chi}_{1}^{0}$ is absolutely stable, and therefore, it is a natural candidate for cold dark matter in the Universe. The singlino $\tilde{S}$ does not couple to fermions. The other two Higgsinos $\tilde{H}_{u, d}^{0}$ couple very weakly to fermions, except for the top quark (and to the bottom quark and the tau lepton to a lesser extent). Consequently, the scattering processes involving (s)fermions of the first and second generations are expected to be dominantly sensitive to the gaugino components of neutralinos.

\section{THE LHC SIGNATURES OF THE $\boldsymbol{U}(1)^{\prime}$ MODEL}

The CMS and the ATLAS experiments at the LHC, a proton-proton collider with center-of-mass energy $\sqrt{s}=$ $14 \mathrm{TeV}$, will be searching for physics beyond the SM. The $U(1)^{\prime}$ model would show up in experiments at the LHC via the $U(1)^{\prime}$ gauge boson and gauge fermion as well as the singlet chiral field in its superpotential. These fermionic and bosonic fields give rise to characteristically distinct yet not necessarily independent signatures at the LHC energies. These effects are discussed and contrasted in this section with the ones in the MSSM by employing the gauge basis instead of the physical (mass-eigenstate) basis, for simplicity and clarity of the discussions.

We first briefly summarize those effects which are genuine to the $U(1)^{\prime}$ model by considering its bosonic sector only. These effects have been studied in detail in the literature [7]; bounds on various model parameters will be tightened as more and more experimental data accumulate. In this work we will not reanalyze these effects, but will take into account the implied constraints.

The bosonic sector of the $U(1)^{\prime}$ model shows up through the $Z^{\prime}$ gauge boson and the singlet Higgs boson $S$. The cleanest and the most direct signal of a $Z^{\prime}$ gauge boson, if accessible at the LHC, will be a new resonance, centered at $M_{\ell \ell}=M_{Z^{\prime}}$, in the dilepton spectrum $(\ell=e$ or $\mu$ unless otherwise stated) $[8,9]$

$$
p p \rightarrow Z^{\prime}+X \rightarrow \ell^{+} \ell^{-}+X .
$$

This proceeds through $q \bar{q}$ annihilation followed by an $s$-channel $Z^{\prime}$ exchange. The existing bounds from LEP [10] and Tevatron [11] require $Z^{\prime}$ to weigh near a $\mathrm{TeV}$ or higher, depending on the details of the model which determine the $Z^{\prime}$ couplings to the quarks and leptons [8].

The extra Higgs boson, $H^{\prime}$ weighs close to $M_{Z^{\prime}}$ and it is typically the heaviest Higgs boson in the spectrum [1214]. The $S$ field (which gives rise to the physical $H^{\prime}$ boson after diagonalization of the Higgs mass-squared matrix) is produced via

$$
p p \rightarrow Z^{\prime}+X \rightarrow S S^{\star}+X,
$$

whereupon the $S$ field subsequently decays into lighter fields in the model:

$$
\begin{array}{lrrr}
S \rightarrow H_{u}^{0} H_{d}^{0}, & H_{u}^{+} H_{d}^{-}, & H_{d}^{0} \tilde{t}_{L} \tilde{t}_{R}^{\star}, & H_{d}^{+} \tilde{b}_{L} \tilde{t}_{R}^{\star}, \\
H_{u}^{0} \tilde{b}_{L} \tilde{b}_{R}^{\star}, & H_{u}^{-} \tilde{t}_{L} \tilde{b}_{R}^{\star}, & H_{u}^{0} \tilde{\ell}_{L} \tilde{\ell}_{R}^{\star}, & H_{u}^{-} \tilde{\nu}_{L} \tilde{\ell}_{R}^{\star} .
\end{array}
$$

The phenomenological implications of these decays have already been analyzed in [13,14].

There are also effects at the LHC which would involve both the $Z^{\prime}$ and the $S$ fields in an interacting fashion. One such process is the Higgs production via the Bjorken 
mechanism

$$
\begin{aligned}
p p & \rightarrow\left(Z, Z^{\prime}\right)+X \\
& \rightarrow\left(Z, Z^{\prime}\right)+C P-\text { even Higgs bosons }+X,
\end{aligned}
$$

which differs from its MSSM counterpart by the presence of both the $Z^{\prime}$ and the $S$ contributions [13]. It is because of these effects, in conjunction with (10), that the Higgs boson discovery limits can be modified significantly in the $U(1)^{\prime}$ model.

\section{$U(1)^{\prime}$ Effects through gauge and Higgs fermions}

The non-MSSM neutral fermions $\tilde{S}$ and $\tilde{Z}^{\prime}$, which mediate the superpartner forces, are part of the neutralino sector (4), and thus, extraction of the $U(1)^{\prime}$ effects from the collider data can also be accomplished via those processes involving the neutralinos. At hadron colliders, such as the LHC, neutralinos $\left(\tilde{\chi}_{i}^{0}, i=1, \ldots, 6\right)$ can be produced directly in pairs or in association with the charginos $\left(\tilde{\chi}_{r}^{+}\right.$, $r=1,2)$, gluinos $\tilde{g}$ or squarks $\tilde{q}[15]$

$$
p p \rightarrow \tilde{\chi}_{i}^{0} \tilde{\chi}_{j}^{0}, \quad \tilde{\chi}_{i}^{0} \tilde{\chi}_{r}^{+}, \quad \tilde{\chi}_{i}^{0} \tilde{g}, \quad \tilde{\chi}_{i}^{0} \tilde{q},
$$

via the $s$-channel gauge boson exchange (the first two channels above) or the $t$-channel squark exchange (all the channels). The trilinear gauge boson couplings are completely antisymmetric for the $S U(2)_{L}$ group and do not exist for the Abelian ones, and hence, $Z$ and $Z^{\prime}$ gauge bosons do not couple to the neutral gauginos $\tilde{W}^{3}, \tilde{B}$ and $\tilde{Z}^{\prime}$. Instead, they couple only to the neutral Higgsinos $\tilde{H}_{u, d}^{0}$ contributing to the $\tilde{\chi}_{i}^{0} \tilde{\chi}_{j}^{0}$ production. On the other hand, the $W^{ \pm}$boson couples to $\tilde{W}^{3} \tilde{W}^{ \pm}$as well as to $\tilde{H}_{u, d}^{0} \tilde{H}_{u, d}^{ \pm}$, and thus, the $s$-channel $W^{ \pm}$exchange gives rise to $\tilde{\chi}_{i}^{0} \tilde{\chi}_{r}^{+}$final states containing both the gauginos and the Higgsinos. In addition, the $Z^{\prime}$ exchange (dominantly $Z_{2}$ exchange for small $Z-Z^{\prime}$ mixing) causes pair-production of the singlino $\tilde{S}$. In fact, this channel is the only mode which leads to $\tilde{S}$ production since the $t$-channel squark exchange produces only the gaugino components of the neutral fermions. In consequence, while the $s$-channel gauge boson exchanges generate the $\tilde{H}_{u, d}^{0}$ and the $\tilde{S}$ components of neutralinos, the $t$-channel squark exchange gives rise to the $\tilde{W}^{3}, \tilde{B}$ as well as the $\tilde{Z}^{\prime}$ components. In this sense, the two amplitudes exhibit complementarity in producing the neutral Higgsinos and the gauginos. Besides, the neutralino mass matrix (5) enables the production of all the neutralino states $\tilde{\chi}_{i}^{0}$, no matter which gaugino or Higgsino component is actually produced at the interaction vertex.

The existing bounds on the $Z^{\prime}$ boson mass [7] do not necessarily imply a suppression of the pair-production processes at the LHC energies, as this cross section may get enhanced due to the resonance effects for the center-ofmass energy near the $Z^{\prime}$ mass. This implies that the singlino pair-production could be as strong as that involving the other two Higginos $\tilde{H}_{u, d}^{0}$.
Once produced, all neutralinos decay into isolated leptons, hard jets (initiated by quarks or gluons), photons and the lightest neutralino $\tilde{\chi}_{1}^{0}$ (which appears as a momentum imbalance or the missing transverse energy $\mathscr{E}_{T}$ in all the SUSY processes since it is the lightest supersymmetric particle (LSP), which is stable due to the conserved $R$ parity) via a chain of cascade decays. The decay patterns of interest, especially those offering clean collider signatures, are the ones which yield isolated leptons. In this sense, a typical cascade decay would look like

$$
\begin{aligned}
(\text { heavy ino }) & \rightarrow(\text { lepton })(\text { slepton })^{*} \\
& \rightarrow(\text { lepton })(\text { anti-lepton })(\text { light ino) }
\end{aligned}
$$

where "ino" stands for any of the neutral or charged gauginos or Higgsinos in the model. Every cascade must necessarily end with the "lightest ino" i.e., the LSP, and therefore, decay chains of this sort proceed through several intermediate steps depending on the mass and the couplings of the mother-ino.

It is highly illustrative to analyze these cascade decays in the Lagrangian basis $\tilde{G}_{a}$, and we do so for the remainder of this section. A precise analysis in the physical basis $\tilde{\chi}_{i}^{0}$, which takes into account the mixings in the neutralino mass matrix (5), will be given in the next section.

The cascade decays (14) are the key processes for determining the sparticle properties from the decay rates and topologies at the LHC [16]. In the MSSM they involve the hypercharge and the isospin gauginos as well as the Higgsinos. In the $U(1)^{\prime}$ model, with the addition of new neutral fermions $\tilde{Z}^{\prime}$ and $\tilde{S}$, the ino decays can acquire certain novel features not present in the MSSM. This point can be exemplified by considering the decay

$$
\tilde{W}^{3} \rightarrow \ell^{+} \tilde{\ell}^{\star-} \rightarrow \ell^{+} \ell^{-} \tilde{B},
$$

which in the MSSM hardly ever extends further since $\tilde{W}^{3}$ and $\tilde{W}^{ \pm}$are nearly mass-degenerate. In fact, the $S U(2)_{L}$ breaking effects that split them in mass turn out to be small so that $\tilde{\chi}_{2}^{0}$ and $\tilde{\chi}_{1}^{ \pm}$have approximately the same mass $[3,16]$. Hence, in the MSSM the decay of $\tilde{W}^{3}$ dominantly gives a dilepton signal. In contrast to this, in the $U(1)^{\prime}$ model, if $\tilde{Z}^{\prime}$ falls in between $\tilde{W}^{3}$ and $\tilde{B}$ in mass, the cascade (15) proceeds through one more step

$$
\begin{aligned}
\tilde{W}^{3} & \rightarrow \ell^{+} \tilde{\ell}^{\star-} \rightarrow \ell^{+} \ell^{-} \tilde{Z}^{\prime} \rightarrow \ell^{+} \ell^{-} \ell^{\prime+} \tilde{\ell}^{\prime \star-} \\
& \rightarrow \ell^{+} \ell^{-} \ell^{\prime+} \ell^{\prime-} \tilde{B},
\end{aligned}
$$

to yield a tetralepton final state. Obviously, this final state also arises when $\tilde{Z}^{\prime}$ is heavier than $\tilde{W}^{3}$. Engineered by the $U(1)^{\prime}$ gaugino, this is one distinctive feature that helps distinguish the $U(1)^{\prime}$ signatures from those of the MSSM.

Unlike the $U(1)^{\prime}$ gaugino, the singlino $\tilde{S}$, since it does not couple to quarks and leptons directly, exhibits a completely different decay pattern, in that the Higgs bosons are always involved in the process. One possible decay chan- 
nel proceeds with the $U(1)^{\prime}$ gaugino

$$
\tilde{S} \rightarrow S \tilde{Z}^{\prime},
$$

where $\tilde{Z}^{\prime}$ decays into leptons and $\tilde{B}$ as described above, and the singlet Higgs $S$ decays into the SM particles via the doublet Higgs fields $H_{u, d}$. The other channel proceeds with the Higgsinos in the decay products,

$$
\tilde{S} \rightarrow H_{u}^{0} \tilde{H}_{d}^{0}, \quad H_{u}^{+} \tilde{H}_{d}^{-},
$$

wherein the Higgs bosons and the fermions follow the usual decay chains until the leptons (possibly also quarks) plus the $\tilde{B}$ state are reached.

The direct pair-production mechanisms in (13) are not the only means of producing neutralinos; moreover, they are not necessarily the dominant ones. Indeed, neutralinos and charginos are produced in cascade decays of the gluinos, squarks and sleptons. At the LHC energies, if accessible kinematically, gluinos and squarks possess the largest production cross section [17] among all the sparticles. Neutralinos or charginos arising from the squark/gluino decays must be much more abundant than from all other sources, and an analysis of these can give critical information about the absence/presence of an extra $U(1)$ group. However, since all the SUSY processes end with a debris containing $\tilde{\chi}_{1}^{0}$, which escapes detection in the detector, a complete reconstruction of the masses and couplings of the sparticles is not possible. Therefore, observability is based on the criterion of having a significant excess of events of a given topology over a predetermined background $[16,18]$. For extracting information on a possible $U(1)^{\prime}$ group, one has to determine the squark/gluino decay channels pertaining to the $U(1)^{\prime}$ model, and compare the signal with the MSSM prediction, as will be done explicitly in the next section.

The gluinos, unlike the $S U(2)_{L} \otimes U(1)_{Y} \otimes U(1)^{\prime}$ gauginos, can be pair-produced via the gluon exchange in the $s$-channel at the LHC energies via

$$
p p \rightarrow \tilde{g} \tilde{g}, \quad \tilde{q} \tilde{q}, \quad \tilde{g} \tilde{q},
$$

through gluon-gluon, gluon-quark and quark-quark scattering [17]. Following their production, gluinos and squarks decay further. If the gluino is heavier than squarks then it decays into a quark and squark $\tilde{q}$

$$
\tilde{g} \rightarrow q \tilde{q},
$$

and subsequently $\tilde{q}$ initiates a series of cascade decays yielding a debris containing jets, isolated leptons and $\tilde{\chi}_{1}^{0}$. On the other hand, if the gluino is lighter than (some of the) squarks then the squark $\tilde{q}$ decays into gluino and quark, and then the gluino decays into lighter squarks and quarks yielding eventually a similar debris. Therefore, the essential features of the model can be extracted by exploring the decay patterns of the squarks. The decay patterns of sfermions, for either chirality, are exhibited in Table I, where the channels in the MSSM and the $U(1)^{\prime}$ model are dis- played in adjacent columns for comparison. As is clear from this table, the effect of the $U(1)^{\prime}$ group is in the opening of a new channel

$$
\tilde{f}_{L, R} \rightarrow f_{L, R} \tilde{Z}_{R, L}^{\prime},
$$

by the emission of the $U(1)^{\prime}$ gaugino. This channel modifies not only the branching ratios of the squarks but also the decay topologies of certain sparticles expected in the MSSM.

For a clearer exposition of the features added by the squark decays into $\tilde{Z}^{\prime}$, we elaborate on the decay channels listed in Table I. The squarks of the first and second generations possess the following properties: (i) the mass and gauge eigenstates (especially for the scalar up and down quarks) are identical due to their exceedingly small Yukawa couplings, (ii) the flavor and the gauge eigenstates of the scalar up and down quarks are identical whereas the scalar strange quark might possesses significant flavor mixing with the scalar bottom quark, (iii) they do not exhibit any appreciable coupling to the Higgsinos but only to the gauginos, and (iv) they turn out to be the heaviest scalars of approximately the same mass, nearly mass degenerate with the gluino, in the minimal supergravity [3]. In the light of these features, these squarks provide a perfect playground for probing the gaugino sector (and hence the extended gauge structures) with a conservative number of SUSY parameters (no direct dependence on the $\mu$ parameter and trilinear couplings, and a weak dependence on $\tan \beta$ via $D$-term contributions).

In contrast to the squarks in the first and second generations, the squarks of the third generation exhibit nonnegligible couplings to Higgs bosons and fermions, and hence, all the decay modes in Table I become relevant for them. Besides, they necessarily exhibit sizable left-right mixings causing mass-eigenstate squarks to have significant mass splitting [12]. Moreover, at least in the minimal supergravity, the third-generation squarks, especially the

TABLE I. The decay channels of the scalar fermions $\tilde{f}$ in the MSSM and the $U(1)^{\prime}$ model. The couplings to Higgsinos $\tilde{H}_{f}^{ \pm}$and $\tilde{H}_{f}^{0}\left(\equiv \tilde{H}_{u}^{0}\right.$ for $f=u$ and $\equiv \tilde{H}_{d}^{0}$ for $\left.f=d, \ell\right)$ are important only for the fermions in the third generation, in particular, the top quark. As follows from (1), the singlino $\tilde{S}$ does not couple to fermions directly, and thus, the $U(1)^{\prime}$ couplings enter via the decays into $\tilde{Z}^{\prime}$ only.

\begin{tabular}{lcc}
\hline \hline Sfermion & MSSM & $U(1)^{\prime}$ Model \\
\hline$\tilde{f}_{R}$ & $\tilde{f}_{R} \rightarrow f_{R} \tilde{B}$ & $\tilde{f}_{R} \rightarrow f_{R} \tilde{B}_{R}$ \\
& $\tilde{f}_{R} \rightarrow f_{L} \tilde{H}_{f}^{0}$ & $\tilde{f}_{R} \rightarrow f_{L} \tilde{H}_{f}^{0} \oplus \tilde{f}_{R} \rightarrow f_{R} \tilde{Z}^{\prime}$ \\
$\tilde{f}_{R} \rightarrow f_{L}^{\prime} \tilde{H}_{f}^{ \pm}$ & $\tilde{f}_{R} \rightarrow f_{L}^{\prime} \tilde{H}_{f}^{ \pm}$ \\
$\tilde{f}_{L}$ & $\tilde{f}_{L} \rightarrow f_{L} \tilde{B}$ & $\tilde{f}_{L} \rightarrow f_{L} \tilde{B}^{-}$ \\
& $\tilde{f}_{L} \rightarrow f_{L} \tilde{W}^{3}$ & $\tilde{f}_{L} \rightarrow f_{L} \tilde{W}^{3}$ \\
& $\tilde{f}_{L} \rightarrow f_{L}^{\prime} \tilde{W}^{ \pm}$ & $\tilde{f}_{L} \rightarrow f_{L}^{\prime} \tilde{W}^{ \pm} \oplus \tilde{f}_{L} \rightarrow f_{L}$ \\
& $\tilde{f}_{L} \rightarrow f_{R} \tilde{H}_{f}^{0}$ & $\tilde{Z}_{L}^{\prime} \tilde{f}_{L} \rightarrow f_{R} \tilde{H}_{f}^{0}$ \\
& $\tilde{f}_{L} \rightarrow f_{R}^{\prime} \tilde{H}_{f}^{ \pm}$ & $\tilde{f}_{L} \rightarrow f_{R}^{\prime} \tilde{H}_{f}^{ \pm}$ \\
\hline \hline
\end{tabular}


stops, turn out to weigh well below the ones in the first and second generations thanks to the counterbalancing effect of the rise in the squark mass due to the Yukawa couplings [3]. Because of these features, the third-generation squarks involve a larger set of SUSY parameters than the first- and second-generation ones, and therefore, they enable exploration of various parameters, like the trilinear couplings and the $\mu$ parameter, not possible with the first- and second-generation squarks. In this work we will not explore the third-generation squarks any further. They are in principle distinguishable by their decay products-the top and bottom quarks can be tagged at the LHC experiments with good efficiency. While their exploration would give important information about various SUSY parameters, and especially, on the Higgs/Higgsino sectors, for the purpose of disentangling the imprints of the extra gauge symmetries in experimental data, the squarks in the first and second generations would suffice.

As a highlighting case study, we start with the analysis of the decay patterns of the first- or the second-generation right-handed squark. From Table I it is clear that, in the MSSM, a right-handed squark $\tilde{q}_{R}$, with no gauge quantum number other than color and hypercharge, possesses one single decay channel

$$
\tilde{q}_{R} \rightarrow q_{R} \tilde{B},
$$

which uniquely leads to 1 jet +0 lepton $+\mathscr{E}_{T}$ signal if the bino $\tilde{B}$ is the LSP. If bino is not the LSP, then it further decays into $\tilde{\chi}_{1}^{0}$ emitting at least one dilepton $\ell^{+} \ell^{-}$[16]. In either case, the decay mode above has $100 \%$ branching fraction as there is no other open decay channel for the $\tilde{q}_{R}$ in the MSSM.

In contrast to the MSSM decay mode (22), the righthanded squarks exhibit a completely new decay pattern in the $U(1)^{\prime}$ model. As seen from Table I, $\tilde{q}_{R}$ now decays via two distinct channels,

$$
\tilde{q}_{R} \rightarrow q_{R} \tilde{B}, \quad \tilde{q}_{R} \rightarrow q_{R} \tilde{Z}^{\prime},
$$

so that the branching ratio into $\tilde{B}$ is no longer $100 \%$. A rough estimate gives

$$
\begin{aligned}
\mathcal{B}^{U(1)^{\prime}}\left(\tilde{q}_{R} \rightarrow q_{R} \tilde{B}\right) \simeq & \frac{g_{Y}^{2} Y_{q_{R}}^{2}}{g_{Y}^{2} Y_{q_{R}}^{2}+g_{Y^{\prime}}^{2} Y_{q_{R}}^{\prime 2}} \\
& <\mathcal{B}^{M S S M}\left(\tilde{q}_{R} \rightarrow q_{R} \tilde{B}\right)=1,
\end{aligned}
$$

where, realistically, gauginos are taken to be light $m_{\tilde{B}}$, $m_{\tilde{Z}^{\prime}} \ll m_{\tilde{q}_{R}}$, and various mixings encoded in the neutralino mass matrix (5) are neglected for simplicity. This estimate reveals that the gauge fermion $\tilde{Z}^{\prime}$ of the $U(1)^{\prime}$ group modifies the decay properties of the right-handed squarks in a way that can be probed by a measurement of the squark branching ratio.

However, the branching fraction is not the whole story. Indeed, depending on the nature of the LSP, one can make further observations which could be of crucial importance for the searches for an extra $U(1)$ group at the LHC. Below, we elaborate on several distinct possibilities:

(i) Bino LSP: In this case, in the MSSM, right-handed squarks with light fermionic partners decay only hadronically as in (22). The resulting 0 lepton + 1 jet $+\mathbb{E}_{T}$ signal can be unambiguously established at the LHC [16].

The situation in the $U(1)^{\prime}$ model is strikingly different than in the MSSM. Decays into the $\tilde{B}$ yield purely hadronic states as in the MSSM. However, decays into the $\tilde{Z}^{\prime}$ give rise to a chain of cascade decays depending on how heavy $\tilde{Z}^{\prime}$ is compared to other gauginos. While the first decay channel in (23) still generates a 0 lepton +1 jet $+\mathbb{E}_{T}$ signal of relative amount (24), the second channel in (23) gives rise to the final states containing at least two oppositely-charged leptons. One can have dileptons

$$
\tilde{q}_{R} \rightarrow q_{R} \tilde{Z}^{\prime} \rightarrow q_{R} \ell^{+} \tilde{\ell}^{\star-} \rightarrow q_{R} \ell^{+} \ell^{-} \tilde{B},
$$

or tetraleptons

$$
\begin{aligned}
\tilde{q}_{R} & \rightarrow q_{R} \tilde{Z}^{\prime} \rightarrow q_{R} \ell^{+} \tilde{\ell}^{\star-} \rightarrow q_{R} \ell^{+} \ell^{-} \tilde{W}^{3} \\
& \rightarrow q_{R} \ell^{+} \ell^{-} \ell^{\prime+} \tilde{\ell}^{\prime-} \rightarrow q_{R} \ell^{+} \ell^{-} \ell^{\prime+} \ell^{\prime-} \tilde{B},
\end{aligned}
$$

in the final state. Sleptons in the intermediate states couple to gauginos and leptons via the modes listed in Table I.

Thus, when the LSP is dominated by bino (which is what happens in most of the parameter space [6]), a prime signature of a $U(1)^{\prime}$ extension of the MSSM is the reduction of purely hadronic events originating from the decays (22) and a corresponding enhancement of the leptonic events via the decays (25) and (26). While the rates of these decays and the depletion in the number of purely hadronic events depend on the masses and couplings of the intermediate sparticles in the cascades, the leptonic final states stemming from the right-handed squarks should offer sufficiently clean signatures to establish the existence of a $U(1)^{\prime}$ extension at the LHC.

(ii) Zino-prime LSP: In this case, mainly the roles of the $\tilde{B}$ and $\tilde{Z}^{\prime}$ are interchanged in terms of hadronic/ leptonic contents of the decay products. In particular, while the second decay channel in (23) leads to purely hadronic events, the first one gives rise to the leptonic final states similar to (25) and (26). In this scenario, an interesting point is that the squark decays through the $U(1)^{\prime}$ gaugino lead to nonleptonic 1 jet $+\mathbb{E}_{T}$ final states.

(iii) Oblique LSP: In general, the LSP does not need to be overwhelmed by a single gaugino and Higgsino component. Indeed, existing bounds on the relic density of dark matter particles can be satisfied with an LSP candidate comprised of various neutral fermions. While in the $U(1)^{\prime}$ model under study, the 
LSP is dominated by the bino component in most of the parameter space [6], depending on the dominant compositions of the LSP, a given decay mode, as listed in Table I, may or may not exhibit a chain of cascades ending preferably with leptons.

The above considerations show that the decay patterns of the right-handed squarks in the first and the second generations would prove to be sensitive probes of gauge extensions of the MSSM under which right-handed quark fields are charged.

The decay characteristics of the left-handed squarks differ from those of the right-handed squarks due to their $S U(2)_{L}$ quantum number. Indeed, as shown in Table I, the left-handed squarks decay not only into the bino but also into the charged and neutral winos. Therefore, a lefthanded squark, in a bino LSP scenario, can yield a 0 lepton +1 jet $+\mathbb{E}_{T}$ final state via its decay into $\tilde{B}$ as in (22), as well as the final states with 1 jet $+\mathscr{E}_{T}$ plus at least one charged lepton. The main impact of the decays into $\tilde{Z}^{\prime}$ depends on the $\tilde{Z}^{\prime}$ mass, increasing the length of the cascade.

Nonetheless, even in the left-handed fermion sector, there are still interesting patterns for which the MSSM and the $U(1)^{\prime}$ model exhibit striking differences. For example, consider the single-lepton production mode:

$$
\tilde{q}_{L} \rightarrow q_{L}^{\prime} \tilde{W}^{ \pm} \rightarrow q_{L}^{\prime} \ell^{ \pm} \tilde{\nu}_{\ell}^{\star} \rightarrow q_{L}^{\prime} \ell^{ \pm} \bar{\nu}_{\ell} \tilde{B},
$$

wherein the missing energy comprises both the bino and the neutrino emissions. Since $\tilde{W}^{ \pm}$and $\tilde{W}^{3}$ are nearly degenerate in mass, this cascade hardly extends any further in the MSSM. In the $U(1)^{\prime}$ model, however, if $\tilde{Z}^{\prime}$ lies below $\tilde{W}^{3}$ and above $\tilde{B}$ then the decay chain (27) proceeds one step further

$$
\begin{aligned}
\tilde{q}_{L} & \rightarrow q_{L}^{\prime} \tilde{W}^{ \pm} \rightarrow q_{L}^{\prime} \ell^{ \pm} \tilde{\nu}_{\ell}^{\star} \rightarrow q_{L}^{\prime} \ell^{ \pm} \bar{\nu}_{\ell} \tilde{Z}^{\prime} \rightarrow q_{L}^{\prime} \ell^{ \pm} \bar{\nu}_{\ell} \ell^{\prime+} \tilde{\ell}^{\prime+} \\
& \rightarrow q_{L}^{\prime} \ell^{ \pm} \bar{\nu}_{\ell} \ell^{\prime+} \ell^{\prime-} \tilde{B},
\end{aligned}
$$

yielding a trilepton signal. This $U(1)^{\prime}$ result is strikingly different from the one in the MSSM where the trilepton signal is expected to be suppressed, if not completely blocked.

If the LSP is not the bino but the $\tilde{Z}^{\prime}$, then essentially the roles of (27) and (28) are interchanged. A $\tilde{Z}^{\prime}$ LSP has the same features mentioned while discussing the $\tilde{q}_{R}$ decays. For a Higgsino LSP decay, (27) gains further steps yielding additional lepton pairs.

Summarizing this subsection, we have investigated the collider signatures of the $U(1)^{\prime}$ group in the cascade decays of the first and second generations of scalar quarks. This extra gauge symmetry offers various collider signatures by modifying the rates, topologies and the pattern of various decay modes. The $U(1)^{\prime}$ gaugino $\tilde{Z}^{\prime}$ and the singlino $\tilde{S}$ are the avatars of the $U(1)^{\prime}$ model. The discussions have been based on the Lagrangian-basis inos $\tilde{G}^{a}$ for a clear tracking of various effects. An accurate analysis must necessarily take into account the physical, mass-eigenstate neutral fermions $\tilde{\chi}_{i}^{0}$ as well as the mass-eigenstate sfermions (mainly the ones in the third generation). This will undertaken in the next section in numerical studies of the squark decays.

\section{THE LHC SIGNALS OF THE $\boldsymbol{U}(1)^{\prime}$ MODEL}

In this section we perform a simulation study of the scattering processes indicative of the additional $U(1)^{\prime}$ group. In particular, we analyze the decay patterns of the scalar quarks in order to determine their rates, topologies and signatures by explicitly working with the physical neutralinos, squarks and sleptons.

The $U(1)^{\prime}$ model consists of a number of parameters not yet specified by experiments. In order to make realistic numerical estimates of the processes discussed in the previous section, one has to adopt a set of viable parameters, compatible with the existing bounds from various sources. To this end, the following parameter choices will be used in the numerical analysis:

(i) The first group of unknown parameters refers to the $U(1)^{\prime}$ charges of the fields. All the properties of the $U(1)^{\prime}$ model advocated so far hold for a generic charge assignment. For the numerical analysis, we assume the $G_{\mathrm{SM}} \otimes U(1)^{\prime}$ models to be descending from SUSY GUTs which provide the absence of anomalies and several other well-studied features [1]. The breaking pattern,

$$
\begin{aligned}
E_{6} & \rightarrow S O(10) \otimes U(1)_{\psi} \rightarrow S U(5) \otimes U(1)_{\chi} \otimes U(1)_{\psi} \\
& \rightarrow G_{\mathrm{SM}} \otimes U(1)_{Y^{\prime}}^{\prime},
\end{aligned}
$$

gives rise to the $G_{\mathrm{SM}} \otimes U(1)^{\prime}$ model of interest from the $E_{6}$ SUSY GUT. Each arrow in this chain corresponds to spontaneous symmetry breakdown at a specific (presumably ultra high) energy scale. Here, by construction,

$$
U(1)_{Y^{\prime}}=\cos \theta_{E_{6}} U(1)_{\psi}-\sin \theta_{E_{6}} U(1)_{\chi},
$$

and the $U(1)^{\prime}$ invariance is broken near the $\mathrm{TeV}$ scale whereas the other orthogonal combination $U(1)_{Y^{\prime}}^{\prime \prime}=$ $\cos \theta_{E_{6}} U(1)_{\chi}+\sin \theta_{E_{6}} U(1)_{\psi}$ is broken at a much higher scale, not accessible to the LHC experiments. The angle $\theta_{E_{6}}$ designates the breaking direction in $U(1)_{\chi} \otimes U(1)_{\psi}$ space, and it is a function of the gauge couplings and vacuum expectation values (VEVs) associated with the breaking. The $U(1)_{\chi}$ and $U(1)_{\psi}$ charge assignments are shown in Table II. In (30), a low-energy $G_{\mathrm{SM}} \otimes U(1)^{\prime}$ model arises with

$$
Y_{f}^{\prime}=\cos \theta_{E_{6}} Q_{\psi}^{f}-\sin \theta_{E_{6}} Q_{\chi}^{f}, \quad g_{Y^{\prime}}=\sqrt{\frac{5}{3}} g_{Y},
$$


TABLE II. The $U(1)_{\psi}$ and $U(1)_{\chi}$ charges of the superfields. The left side of the table lists the particle spectrum of the $G_{\mathrm{SM}} \otimes$ $U(1)^{\prime}$ model whereas on the right side, the chiral fields $\hat{N}, \hat{D}_{u}$ and $\hat{D}_{d}$ form a sector necessary for canceling the anomalies [19], yet too heavy to leave any significant impact on the LHC experiments [9]. Clearly, $U(1)_{\psi}$ is a viable model for solving the $\mu$ problem of the MSSM but $U(1)_{\chi}$ is not.

\begin{tabular}{lrrrrrrrrrrr}
\hline \hline$\hat{f}$ & $\hat{Q}$ & $\hat{U}$ & $\hat{D}$ & $\hat{L}$ & $\hat{E}$ & $\hat{H}_{d}$ & $\hat{H}_{u}$ & $\hat{S}$ & $\hat{N}$ & $\hat{D}_{u}$ & $\hat{D}_{d}$ \\
\hline $2 \sqrt{6} Q_{\psi}^{f}$ & 1 & 1 & 1 & 1 & 1 & -2 & -2 & 4 & 1 & -2 & -2 \\
$2 \sqrt{10} Q_{\chi}^{f}$ & -1 & -1 & 3 & 3 & -1 & -2 & 2 & 0 & -5 & 2 & -2 \\
\hline \hline
\end{tabular}

for any field $f$ in the spectrum with the breaking determined by the angle $\theta_{E_{6}}$. It is clear that if the $U(1)^{\prime}$ model is to solve the $\mu$ problem of the MSSM, then $Y_{\hat{S}}^{\prime} \neq 0$, and hence, as suggested by Table II, $\theta_{E_{6}}=\pi / 2$ should be avoided.

(ii) The soft-breaking masses shared with the MSSM are assigned the following values:

$m_{\tilde{q}_{L}}=m_{\tilde{q}_{R}}=1200 \mathrm{GeV}, \quad m_{\tilde{e}_{L}}=350 \mathrm{GeV}$,

$m_{\tilde{e}_{R}}=200 \mathrm{GeV}, \quad M_{\tilde{Y}}=100 \mathrm{GeV}$,

$M_{\tilde{W}}=400 \mathrm{GeV}, \quad M_{\tilde{g}}=1300 \mathrm{GeV}$,

where $m_{\tilde{q}_{L, R}}$ and $m_{\tilde{e}_{L, R}}$ stand, respectively, for the soft masses (before $G_{\mathrm{SM}} \otimes U(1)^{\prime}$ breaking) of squarks and sleptons in the first and second generations. These parameter values, as for all others, refer to $\mathrm{TeV}$ scale, and no assumption is made of the universality of gaugino and scalar masses at high scale.

(iii) The parameters pertaining to the $U(1)^{\prime}$ sector are assigned the values (the value of $\mu_{\text {eff }}$ determines the singlet VEV and in turn it determines $M_{Z^{\prime}}$ )

$$
\begin{aligned}
h_{s} & =0.6, \mu_{\mathrm{eff}}=1400 \mathrm{GeV}, \\
\tan \beta & =10, \quad \sin \chi=5 \times 10^{-3}
\end{aligned}
$$

where the value of the kinetic mixing angle $\chi$ follows from its radiative nature $[3,20]$. The ranges of the parameters must be such that the bound $\left|\theta_{Z-Z^{\prime}}\right| \lesssim 10^{-3}[7]$ is respected.

(iv) Among the well-studied $E_{6}$ models [1] we specialize to the one defined by the mixing angle

$$
\theta_{E_{6}}=\arcsin [\sqrt{3 / 8}] \simeq 37.76^{\circ} \text {, }
$$

which corresponds to the $U(1)^{\prime} \equiv U(1)_{\eta}$ model. Experimentally, $M_{Z^{\prime}} \geq 933 \mathrm{GeV}$, [11] though this bound is lower by typically $250 \mathrm{GeV}$ if the decays into sparticles are taken into account [9].

(v) For simplicity and later convenience, we scale the gaugino mass parameters $M_{\tilde{Y}^{\prime}}$ and $M_{\tilde{Y} \tilde{Y}^{\prime}}$ with the hypercharge gaugino mass to define the ratios:

$$
\mathcal{R}_{Y^{\prime}} \equiv \frac{M_{\tilde{Y}^{\prime}}}{M_{\tilde{Y}}}, \quad \mathcal{R}_{Y Y^{\prime}} \equiv \frac{M_{\tilde{Y} \tilde{Y}^{\prime}}}{M_{\tilde{Y}}}
$$

the relevant values of which are sampled according to (36)-(38). In obtaining various numerical results we employ different possibilities for the remaining model parameters:

$$
\begin{aligned}
& \text { Small } U(1)_{Y}-U(1)_{Y^{\prime}} \text { Mixing: } \\
& \qquad\left(\mathcal{R}_{Y^{\prime}}, \mathcal{R}_{Y Y^{\prime}}\right)=(1 / 2,0),(2,0),(6,0),(10,0) .
\end{aligned}
$$

$$
\begin{aligned}
& \text { Medium } U(1)_{Y}-U(1)_{Y^{\prime}} \text { Mixing: } \\
& \left(\mathcal{R}_{Y^{\prime}}, \mathcal{R}_{Y Y^{\prime}}\right)=(0,0),(1 / 2,1 / 2),(2,2),(6,6),(10,10) .
\end{aligned}
$$

$$
\begin{aligned}
& \text { Large } U(1)_{Y}-U(1)_{Y^{\prime}} \text { Mixing: } \\
& \qquad\left(\mathcal{R}_{Y^{\prime}}, \mathcal{R}_{Y Y^{\prime}}\right)=(0,1 / 2),(0,2),(0,6),(0,10)
\end{aligned}
$$

In each case, the $\tilde{Z}^{\prime}$ gaugino falls in different bands in mass and mixing, and, depending on how they compare with those of the electroweak gauginos, various decay chains can close or open, thereby leading to distinct signatures at the LHC, as discussed in Sec. III above, and to distinct predictions in the figures and tables to be given below.

The numerical analysis below will provide a generatorlevel description of the LHC signals of the $U(1)^{\prime}$ model for the parameter values specified above. The choice of the $\eta$ model is in no way better than any other model descending from the $E_{6}$ SUSY GUT. Moreover, one can just adopt a low-energy $U(1)^{\prime}$ model without resorting to the $E_{6}$ framework, at the expense of a much larger set of free parameters. Therefore, the $U(1)_{\eta}$ model adopted here can be regarded as a prototype to get an idea of what physics potentials such models can have at the LHC, compared to the MSSM.

\section{A. Branching fractions of squark decay channels}

In this section we compute the branching fractions of the various decay channels discussed in Sec. III. The branching fractions will eventually determine the relative populations of the final states that constitute the signature space of events to be searched for at the LHC. Essentially, we analyze the decay patterns of the squarks by considering separately the $\tilde{q}_{R}$ and $\tilde{q}_{L}$ squarks in the first and second generations (they are themselves mass and flavor eigenstates, to an excellent approximation). We take the parameter values from (31), (32), and (36)-(38). For each, we compute the branching fractions in the MSSM and in the $U(1)^{\prime}$ model, and display them comparatively in the figures to follow. The figures employ a diagrammatic display structure for a clear understanding of the various branching illustrated by varying $\mathcal{R}_{Y^{\prime}}$ and $\mathcal{R}_{Y Y^{\prime}}$ as in (36)-(38). 
In Table III, we list the neutralino masses both in the MSSM and the $U(1)^{\prime}$ model obtained for the values of $\mathcal{R}_{\tilde{Y}^{\prime}}$ and $\mathcal{R}_{\tilde{Y} \tilde{Y}^{\prime}}$. As seen in this table, variations of these ratios mainly modify the masses of the third and fourth neutralinos. In other words, the MSSM mass spectrum corresponds approximately to the states $\left\{\tilde{\chi}_{1}^{0}, \tilde{\chi}_{2}^{0}, \tilde{\chi}_{5}^{0}, \tilde{\chi}_{6}^{0}\right\}$; the $U(1)^{\prime}$ effects amount to inserting the extra states $\left\{\tilde{\chi}_{3}^{0}, \tilde{\chi}_{4}^{0}\right\}$ into the mass spectrum. The MSSM-like neutralinos are nearly immune to these ratios, except for the cases $\mathcal{R}_{\tilde{Y}^{\prime}}=$ 10 and/or $\mathcal{R}_{\tilde{Y} \tilde{Y}^{\prime}}=10$, for which the mass of the $\tilde{Z}^{\prime}$ and/or its mixing with $\tilde{B}$ exceed the $\tilde{B}$ mass by an order of magnitude. One notices that $M_{\tilde{\chi}_{3}^{0}}$ (in small and medium mixing regimes) and $M_{\tilde{\chi}_{1}^{0}}$ (in medium and large mixing regimes) typically decrease with increasing $\mathcal{R}_{\tilde{Y}^{\prime}}$ and/or $\mathcal{R}_{\tilde{Y} \tilde{Y} /}$. This decrease in $M_{\tilde{\chi}_{3}^{0}}$ and $M_{\tilde{\chi}_{1}^{0}}$ is most sensitively correlated with the corresponding increase in $M_{\tilde{\chi}_{6}^{0}}$.

The nature of a given neutralino state $\tilde{\chi}_{i}^{0}$ is determined by its decomposition into the Lagrangian basis $\left\{\tilde{B}, \tilde{W}^{3}, \tilde{H}_{d}^{0}, \tilde{H}_{u}^{0}, \tilde{S}, \tilde{Z}^{\prime}\right\}$. Depicted in Table IV are the compositions of $\tilde{\chi}_{1}^{0}$ (the LSP), $\tilde{\chi}_{3}^{0}$ and $\tilde{\chi}_{4}^{0}$ for the parameter sets (36)-(38). As suggested by the table, the LSP is overwhelmed by its bino component in the small mixing regime, as in the MSSM and in accord with [6]. Nevertheless, its bino component become approximately equal to its singlino component for large $\mathcal{R}_{\tilde{Y}^{\prime}}$ and/or $\mathcal{R}_{\tilde{Y} \tilde{Y}^{\prime}}$, in the medium and large mixing regimes. This increase in the singlino component implies reduced couplings of the LSP to fermions and sfermions, as discussed in Appendices A and $\mathrm{C}$.

The neutralino states $\tilde{\chi}_{3,4}^{0}$ behave differently than the LSP, as they are, as suggested by Table III, genuine to $U(1)^{\prime}$ model. Indeed, they are overwhelmed by $\tilde{Z}^{\prime}$ and $\tilde{S}$ for all of the small, balanced and large mixing regimes. The exceptions arise for large $\mathcal{R}_{\tilde{Y}^{\prime}}$ and/or $\mathcal{R}_{\tilde{Y} \tilde{Y}^{\prime}}$ values for which $\tilde{\chi}_{3}^{0}$ develops a significant bino component, and $\tilde{\chi}_{4}^{0}$ changes to be Higgsino-dominated. For the large mixing regime, how- ever, also $\tilde{\chi}_{4}^{0}$ obtains a significant bino component as $\mathcal{R}_{\tilde{Y} \tilde{Y}^{\prime}}$ grows. These compositions, as detailed in Table IV, directly influence decay patterns and products of a given neutralino: A sizeable $\tilde{Z}^{\prime}$ component gives rise to novel decay patters described in Sec. III; a sizable $\tilde{S}$ composition halts the cascade as it cannot directly decay into fermions, and, similarly, a sizeable bino component stops the cascade as it dominates $\tilde{\chi}_{1}^{0}$.

Having completed the specification of the neutralino sector, we now turn to the analysis of the scalar quark decays. We compute the branching ratios of the decays

$$
\text { squark } \rightarrow \text { quark }+\tilde{\chi}_{i}^{0},
$$

for each quark chirality and for each of the parameter sets (36)-(38). The results are shown in Figs. 1-3 for $\tilde{q}_{R}$, and Figs. 4-6 for $\tilde{q}_{L}$. As illustrated by the panels (a) of Figs. 13 in the MSSM, a right-handed scalar quark decays dominantly into the LSP since it is overwhelmingly the bino. This feature of the right-handed squarks gives rise to the jets $+\mathbb{E}_{T}$ signal at the LHC. By the same token, a lefthanded gluino decays into two quarks and the LSP, and it thus causes 2 jets $+\mathbb{E}_{T}$ events at the LHC [16]. In the $U(1)^{\prime}$ model the right-handed squarks couple to both the $\tilde{B}$ and $\tilde{Z}^{\prime}$, opening novel decay channels. These features are explicitly depicted in Fig. 1 (small mixing regime), Fig. 2 (medium mixing regime) and Fig. 3 (large mixing regime). As suggested by these figures, the right-handed squarks develop additional decay channels with non-negligible branching fractions. In the small mixing regime of (36), the right-handed squark $\tilde{q}_{R}$ decays not only into $q \tilde{\chi}_{1}^{0}$ but also into $q \tilde{\chi}_{3}^{0}$ (whose branching ratio increases with $\mathcal{R}_{\tilde{Y}^{\prime}}$ ) and $q \tilde{\chi}_{4}^{0}$ (whose branching ratio decreases with $\mathcal{R}_{\tilde{Y}^{\prime}}$ as its mass grows to exceed that of the squark).

In the medium mixing regime of (37), the right-handed squark develops a much larger branching fraction into $q \tilde{\chi}_{3}^{0}$, as shown in Fig. 2. In fact, it reaches the $20 \%$ level when $\mathcal{R}_{\tilde{\tilde{Y}^{\prime}}}=\mathcal{R}_{\tilde{Y_{Y} \tilde{Y}^{\prime}}}=10$. This figure is large enough to suggest

TABLE III. The neutralino mass spectra in the $U(1)^{\prime}$ model for the parameter sets (36)-(38).

\begin{tabular}{|c|c|c|c|c|c|c|}
\hline$\left(\mathcal{R}_{\tilde{Y}^{\prime}}, \mathcal{R}_{\tilde{Y} \tilde{Y}^{\prime}}\right)$ & $M_{\tilde{\chi}_{1}^{0}}$ & $M_{\tilde{\chi}_{2}^{0}}$ & $M_{\tilde{\chi}_{3}^{0}}$ & $M_{\tilde{\chi}_{4}^{0}}$ & $M_{\tilde{\chi}_{5}^{0}}$ & $M_{\tilde{\chi}_{6}^{0}}$ \\
\hline MSSM & $100 \mathrm{GeV}$ & $398 \mathrm{GeV}$ & $\cdots$ & $\cdots$ & $1402 \mathrm{GeV}$ & $1405 \mathrm{GeV}$ \\
\hline$(1 / 2,0)$ & $100 \mathrm{GeV}$ & $398 \mathrm{GeV}$ & $955 \mathrm{GeV}$ & $1007 \mathrm{GeV}$ & $1407 \mathrm{GeV}$ & $1408 \mathrm{GeV}$ \\
\hline$(2,0)$ & $97 \mathrm{GeV}$ & $398 \mathrm{GeV}$ & $885 \mathrm{GeV}$ & $1087 \mathrm{GeV}$ & $1407 \mathrm{GeV}$ & $1408 \mathrm{GeV}$ \\
\hline$(6,0)$ & $97 \mathrm{GeV}$ & $398 \mathrm{GeV}$ & $725 \mathrm{GeV}$ & $1326 \mathrm{GeV}$ & $1407 \mathrm{GeV}$ & $1408 \mathrm{GeV}$ \\
\hline$(10,0)$ & $97 \mathrm{GeV}$ & $398 \mathrm{GeV}$ & $600 \mathrm{GeV}$ & $1407 \mathrm{GeV}$ & $1407 \mathrm{GeV}$ & $1602 \mathrm{GeV}$ \\
\hline$(0,0)$ & $100 \mathrm{GeV}$ & $398 \mathrm{GeV}$ & $980 \mathrm{GeV}$ & $982 \mathrm{GeV}$ & $1407 \mathrm{GeV}$ & $1408 \mathrm{GeV}$ \\
\hline$(1 / 2,1 / 2)$ & $100 \mathrm{GeV}$ & $398 \mathrm{GeV}$ & $957 \mathrm{GeV}$ & $1008 \mathrm{GeV}$ & $1407 \mathrm{GeV}$ & $1408 \mathrm{GeV}$ \\
\hline$(2,2)$ & $97 \mathrm{GeV}$ & $398 \mathrm{GeV}$ & $905 \mathrm{GeV}$ & $1107 \mathrm{GeV}$ & $1407 \mathrm{GeV}$ & $1408 \mathrm{GeV}$ \\
\hline$(6,6)$ & $77 \mathrm{GeV}$ & $398 \mathrm{GeV}$ & $876 \mathrm{GeV}$ & $1405 \mathrm{GeV}$ & $1407 \mathrm{GeV}$ & $1497 \mathrm{GeV}$ \\
\hline$(10,10)$ & $54 \mathrm{GeV}$ & $398 \mathrm{GeV}$ & $960 \mathrm{GeV}$ & $1407 \mathrm{GeV}$ & $1407 \mathrm{GeV}$ & $1998 \mathrm{GeV}$ \\
\hline$(0,1 / 2)$ & $100 \mathrm{GeV}$ & $398 \mathrm{GeV}$ & $982 \mathrm{GeV}$ & $983 \mathrm{GeV}$ & $1407 \mathrm{GeV}$ & $1408 \mathrm{GeV}$ \\
\hline$(0,2)$ & $97 \mathrm{GeV}$ & $398 \mathrm{GeV}$ & $1000 \mathrm{GeV}$ & $1002 \mathrm{GeV}$ & $1407 \mathrm{GeV}$ & $1408 \mathrm{GeV}$ \\
\hline$(0,6)$ & $76 \mathrm{GeV}$ & $398 \mathrm{GeV}$ & $1141 \mathrm{GeV}$ & $1159 \mathrm{GeV}$ & $1407 \mathrm{GeV}$ & $1409 \mathrm{GeV}$ \\
\hline$(0,10)$ & $53 \mathrm{GeV}$ & $398 \mathrm{GeV}$ & $1382 \mathrm{GeV}$ & $1391 \mathrm{GeV}$ & $1407 \mathrm{GeV}$ & $1437 \mathrm{GeV}$ \\
\hline
\end{tabular}


TABLE IV. The components of $\tilde{\chi}_{1}^{0}$ (the LSP), $\tilde{\chi}_{3}^{0}$ and $\tilde{\chi}_{4}^{0}$ in the Lagrangian basis $\left\{\tilde{B}, \tilde{W}^{3}, \tilde{H}_{d}^{0}, \tilde{H}_{u}^{0}, \tilde{S}\right.$, $\left.\tilde{Z}^{\prime}\right\}$ for the parameter sets $(36)-$ (38).

\begin{tabular}{|c|c|c|c|c|c|c|c|}
\hline$\underline{\left(\mathcal{R}_{\tilde{Y^{\prime}}}, \mathcal{R}_{\tilde{Y} \tilde{Y}^{\prime}}\right)}$ & $\tilde{\chi}_{1,3,4}^{0}$ & $\tilde{B}$ & $\tilde{W}^{3}$ & $\tilde{H}_{d}^{0}$ & $\tilde{H}_{u}^{0}$ & $\tilde{S}$ & $\tilde{Z}^{\prime}$ \\
\hline \multirow[t]{3}{*}{ MSSM } & $\left(\tilde{\chi}_{1}^{0}\right)_{\mathrm{MSSM}}\left(\sim \tilde{\chi}_{1}^{0}\right)$ & 0.99 & -0.0044 & 0.019 & 0.026 & $\cdots$ & $\cdots$ \\
\hline & $\left(\tilde{\chi}_{3}^{0}\right)_{\operatorname{MSSM}}\left(\sim \tilde{\chi}_{5}^{0}\right)$ & 0.032 & -0.064 & -0.71 & -0.70 & $\cdots$ & $\cdots$ \\
\hline & $\left(\tilde{\chi}_{4}^{0}\right)_{\operatorname{MSSM}}\left(\leadsto \tilde{\chi}_{6}^{0}\right)$ & -0.0084 & 0.029 & -0.71 & 0.71 & $\cdots$ & $\cdots$ \\
\hline \multirow[t]{3}{*}{$(1 / 2,0)$} & $\tilde{\chi}_{1}^{0}$ & -0.99 & 0.0023 & -0.032 & 0.0054 & -0.0004 & -0.0033 \\
\hline & $\tilde{\chi}_{3}^{0}$ & -0.0023 & 0.0038 & 0.021 & 0.067 & -0.71 & 0.70 \\
\hline & $\tilde{\chi}_{4}^{0}$ & 0.0031 & -0.0073 & -0.0042 & 0.055 & -0.70 & -0.71 \\
\hline \multirow[t]{3}{*}{$(2,0)$} & $\tilde{\chi}_{1}^{0}$ & 0.99 & -0.0023 & 0.032 & -0.0054 & -0.0001 & 0.0033 \\
\hline & $\tilde{\chi}_{3}^{0}$ & -0.0025 & 0.004 & 0.019 & 0.066 & -0.74 & 0.67 \\
\hline & $\tilde{\chi}_{4}^{0}$ & 0.0029 & -0.0065 & 0.0065 & 0.055 & -0.67 & -0.74 \\
\hline \multirow[t]{3}{*}{$(6,0)$} & $\tilde{\chi}_{1}^{0}$ & 0.99 & -0.0022 & 0.032 & -0.0053 & -0.0014 & 0.0032 \\
\hline & $\tilde{\chi}_{3}^{0}$ & -0.0031 & 0.0046 & 0.015 & 0.067 & -0.80 & 0.59 \\
\hline & $\tilde{\chi}_{4}^{0}$ & -0.0033 & -0.0071 & 0.037 & -0.079 & 0.59 & 0.80 \\
\hline \multirow[t]{3}{*}{$(10,0)$} & $\tilde{\chi}_{1}^{0}$ & 0.99 & -0.0022 & 0.032 & -0.0052 & -0.0026 & 0.0030 \\
\hline & $\tilde{\chi}_{3}^{0}$ & -0.0038 & 0.0053 & 0.013 & 0.068 & -0.85 & 0.52 \\
\hline & $\tilde{\chi}_{4}^{0}$ & -0.018 & 0.028 & 0.71 & 0.71 & 0.063 & -0.008 \\
\hline \multirow[t]{3}{*}{$(0,0)$} & $\tilde{\chi}_{1}^{0}$ & 0.99 & -0.0023 & 0.032 & -0.0054 & 0.00057 & 0.0034 \\
\hline & $\tilde{\chi}_{3}^{0}$ & 0.0023 & -0.0038 & -0.022 & -0.067 & 0.70 & -0.71 \\
\hline & $\tilde{\chi}_{4}^{0}$ & 0.0032 & -0.0077 & -0.0036 & 0.056 & -0.71 & -0.71 \\
\hline \multirow[t]{3}{*}{$(1 / 2,1 / 2)$} & $\tilde{\chi}_{1}^{0}$ & -0.99 & 0.0013 & -0.032 & 0.0016 & 0.051 & 0.0018 \\
\hline & $\tilde{\chi}_{3}^{0}$ & -0.035 & 0.0037 & 0.020 & 0.066 & -0.71 & 0.70 \\
\hline & $\tilde{\chi}_{4}^{0}$ & 0.036 & 0.0076 & 0.0069 & -0.057 & 0.70 & 0.72 \\
\hline \multirow[t]{3}{*}{$(2,2)$} & $\tilde{\chi}_{1}^{0}$ & 0.98 & 0.0016 & 0.032 & 0.0094 & -0.20 & -0.016 \\
\hline & $\tilde{\chi}_{3}^{0}$ & 0.14 & -0.0037 & -0.013 & -0.062 & 0.73 & -0.67 \\
\hline & $\tilde{\chi}_{4}^{0}$ & -0.14 & -0.0076 & -0.020 & 0.066 & -0.65 & -0.74 \\
\hline \multirow[t]{3}{*}{$(6,6)$} & $\tilde{\chi}_{1}^{0}$ & -0.86 & -0.0075 & -0.030 & -0.033 & 0.51 & 0.036 \\
\hline & $\tilde{\chi}_{3}^{0}$ & -0.38 & 0.0032 & -0.00064 & 0.051 & -0.069 & 0.62 \\
\hline & $\tilde{\chi}_{4}^{0}$ & -0.039 & -0.061 & -0.69 & 0.70 & -0.051 & -0.14 \\
\hline \multirow[t]{3}{*}{$(10,10)$} & $\tilde{\chi}_{1}^{0}$ & 0.72 & 0.011 & 0.027 & 0.048 & -0.70 & -0.035 \\
\hline & $\tilde{\chi}_{3}^{0}$ & -0.55 & 0.0023 & -0.012 & 0.037 & -0.60 & 0.58 \\
\hline & $\tilde{\chi}_{4}^{0}$ & -0.021 & 0.028 & 0.71 & 0.71 & 0.054 & 0.0043 \\
\hline \multirow[t]{3}{*}{$(0,1 / 2)$} & $\tilde{\chi}_{1}^{0}$ & -0.99 & 0.0013 & -0.031 & 0.0016 & 0.051 & 0.0018 \\
\hline & $\tilde{\chi}_{3}^{0}$ & -0.035 & 0.0037 & 0.021 & 0.066 & -0.70 & 0.71 \\
\hline & $\tilde{\chi}_{4}^{0}$ & 0.037 & 0.0079 & 0.0062 & -0.057 & 0.70 & 0.71 \\
\hline \multirow[t]{3}{*}{$(0,2)$} & $\tilde{\chi}_{1}^{0}$ & 0.98 & 0.0017 & 0.032 & 0.0097 & -0.20 & -0.016 \\
\hline & $\tilde{\chi}_{3}^{0}$ & 0.13 & -0.0035 & -0.016 & -0.062 & 0.69 & -0.71 \\
\hline & $\tilde{\chi}_{4}^{0}$ & -0.15 & -0.0085 & -0.015 & 0.063 & -0.69 & -0.71 \\
\hline \multirow[t]{3}{*}{$(0,6)$} & $\tilde{\chi}_{1}^{0}$ & 0.85 & 0.0078 & 0.030 & 0.035 & -0.52 & -0.037 \\
\hline & $\tilde{\chi}_{3}^{0}$ & -0.35 & 0.002 & 0.0031 & 0.048 & -0.61 & 0.71 \\
\hline & $\tilde{\chi}_{4}^{0}$ & 0.39 & 0.0096 & 0.050 & -0.087 & 0.59 & 0.70 \\
\hline \multirow[t]{3}{*}{$(0,10)$} & $\tilde{\chi}_{1}^{0}$ & -0.70 & -0.011 & -0.026 & -0.050 & 0.71 & 0.035 \\
\hline & $\tilde{\chi}_{3}^{0}$ & 0.48 & -0.0021 & 0.097 & -0.057 & -0.51 & 0.71 \\
\hline & $\tilde{\chi}_{4}^{0}$ & -0.030 & -0.052 & -0.57 & 0.59 & -0.26 & -0.42 \\
\hline
\end{tabular}

that this parameter regime should be explored further, as will be done in the next subsection. For the large mixing regime of (38), the branching fraction of the decays into $q \tilde{\chi}_{3}^{0}$ decreases with increasing $\mathcal{R}_{\tilde{Y} \tilde{Y}^{\prime}}$, and, as seen from Fig. 3, eventually vanishes when the decay channel is closed kinematically at $\mathcal{R}_{\tilde{Y} \tilde{Y}^{\prime}}=10$. This extreme is indistinguishable from the MSSM case, shown in panel (a). This is expected since all the neutralinos but $\tilde{\chi}_{1}^{0}$ become too heavy to be produced on-shell by the squark decay.
These figures make it clear that, in the $U(1)^{\prime}$ model, the right-handed squarks can decay into neutralinos other than the LSP. This feature guarantees that, unlike the purely hadronic events 0 lepton + jets $+\mathbb{E}_{T}$ expected in the MSSM, in the $U(1)^{\prime}$ model hadronic as well as leptonic events are initiated by the right-handed squarks. This property, which will be analyzed in detail in the next subsection, is a golden mode to discover such extensions. One also notes that the branchings of $\tilde{q}_{R}$ significantly differ 
from that in the MSSM only in the medium mixing regime, that is, the parameter set (37). In addition, the large mixing regime of (38), becomes indistinguishable from the MSSM case at large $\mathcal{R}_{\tilde{Y} \tilde{Y} /}$. As illustrated by panels (a) of Figs. 4-6 in the MSSM, a left-handed scalar quark decays dominantly into quark plus the lighter chargino $\tilde{\chi}_{1}^{ \pm}$or quark plus the next-to-lightest neutralino $\tilde{\chi}_{2}^{0}$. Therefore, the lefthanded scalar quarks, as analyzed in Sec. III and listed in Table I, give rise to leptonic final states abundantly. The pure hadronic final states are rather rare [16].

Table III shows that the mass of $\tilde{\chi}_{2}^{0}$ remains stuck to its MSSM value, to an excellent approximation. The lighter chargino, which is $\tilde{W}^{ \pm}$dominated, is not expected to deviate from its MSSM mass. Consequently, the $U(1)^{\prime}$ effects are not expected to cause dramatic changes from the branching fractions of $\tilde{q}_{L}$ in the MSSM. This is seen to be the case from Figs. 4-6 corresponding to small, medium and large mixings among $U(1)_{Y}$ and $U(1)_{Y}^{\prime}$ gauginos, respectively, clearly showing that the decay channels of the left-handed squarks are nearly immune to the $U(1)^{\prime}$ effects. The conclusion from this subsection is that the $U(1)^{\prime}$-effects become visible mainly in the fermionic decays of the right-handed scalar quarks, but not in the lefthanded ones. The medium mixing regime of (37) stands as a particularly promising parameter domain for hunting the $U(1)^{\prime}$ effects.

\section{B. The LHC signatures of the $\boldsymbol{U}(\mathbf{1})^{\prime}$ model through jets + leptons $+\mathscr{E}_{T}$ events}

Having computed the squark branching ratios in the previous section, we now turn to the analysis of various final states to be searched for by the ATLAS and CMS experiments at the LHC. We perform a simulation study of a number of LHC events for the MSSM and the $U(1)^{\prime}$ model in a comparative fashion. The scattering processes of interest have the generic form

$$
p p \rightarrow X+\text { SIGNAL, }
$$

where SIGNAL stands for the particular final state characterizing the event. An optimal coverage of the events for which the MSSM and the $U(1)^{\prime}$ model can exhibit striking differences are classified in Table V. We compute the cross sections and branching ratios, and generate parton-level events by using CalcHEP v.2.5 [21]. We modified the package to incorporate the features pertaining to the $U(1)^{\prime}$ model with the help of LanHEP Package [22]. Hadronization (including initial and final state radiations) and restrictions imposed by various cuts have been achieved with PYTHIA [23] by using the CalcHEP-PYTHIA interface. The parton distributions in the proton have been parametrized by using CTEQ6L of LHAPDF. The number of events are calculated for an integrated luminosity $\mathcal{L}=100 \mathrm{fb}^{-1}$, for which the LHC has a sensitivity to the squark and gluino masses around $2.5 \mathrm{TeV}$ [16]. Our goal here is to determine how the MSSM and the $U(1)^{\prime}$ model differ in their predictions for the signals in Table $\mathrm{V}$, driven by the presence of the extra gauge and Higgs fermions. A detailed background analysis is not warranted in this work since its main goal is to compare the MSSM and the $U(1)^{\prime}$ model predictions for the signal events under consideration. Nonetheless, as a set of generic cuts for revealing "new physics" effects (compared to the SM ones), we select only those events satisfying the following restrictions:

(i) Each charged lepton in the final state must have a transverse momentum $p_{T}^{\ell}>15 \mathrm{GeV} / c$.

(ii) Each jet must have a transverse momentum $p_{T}^{\text {jet }}>$ $20 \mathrm{GeV} / c$.

(iii) The missing transverse energy must satisfy $\mathbb{E}_{T} \geq$ $100 \mathrm{GeV}$.

(iv) The particles at the final state propagate in the transverse direction so that the pseudorapidity stays in the interval $-2 \leq \eta \leq 2$.

(v) The initiator energy of jets is $2 \mathrm{GeV}$.

(vi) Two jetted showers of particles are taken to be two distinct jets if their spatial separation satisfies $\Delta R_{j j}>0.7$.

We now perform a full generator-level analysis of the events tabulated in Table $\mathrm{V}$ by taking into account the generation and decays of all the squarks in the first and second generations as well as the gluino via the $p p$ scatterings in (40). We use the Feynman rules in Appendix D, compute the populations of the events in Table V, and plot the results against various observables of interest at the LHC. The analysis performs a comparative study between the MSSM and the $U(1)^{\prime}$ model in regard to their predictions for the processes in Table V. Concerning the parameter choice, we take the $U(1)^{\prime}$ model to be in the medium mixing regime of (37), and consider the two points

$$
\left(\mathcal{R}_{\tilde{Y}}, \mathcal{R}_{\tilde{Y^{\prime} \tilde{Y}^{\prime}}}\right)=(0,0) \quad \text { and } \quad(10,10),
$$

in all the figures that follow. These two points are picked up on the basis of highlighting the $U(1)^{\prime}$ effects in comparison to those of the MSSM.

Among the signals listed in Table $\mathrm{V}$, the signal $3 \ell+$ 2 jet $+\mathbb{E}_{T}$ (SIGNAL 4A), where all leptons originate from the same branch, is not considered further in the numerical analysis. This is due to the fact that this signal requires a decay chain like in Eq. (28) and since we use narrow-width approximation, the scalar neutrino $\tilde{\nu}_{\ell}$ (taken to be relatively light) has to decay through a 4-body decay $\tilde{\nu}_{\ell} \rightarrow$ $\bar{\nu}_{\ell} \ell^{\prime+} \ell^{\prime-} \tilde{B}$ with a tiny branching ratio. Thus, the signal will be much suppressed as compared with the others. This observation is consistent with the region of the parameter space considered here, since for instance, scalar neutrinos heavier than $\tilde{\chi}_{2}^{0}$ and scalar leptons would make it competitive with the others.

The observables with respect to which we analyze the number of events are as follows:

(i) The number of jets $N_{\text {jets }}$ with bin size $=1 \mathrm{GeV}$, 

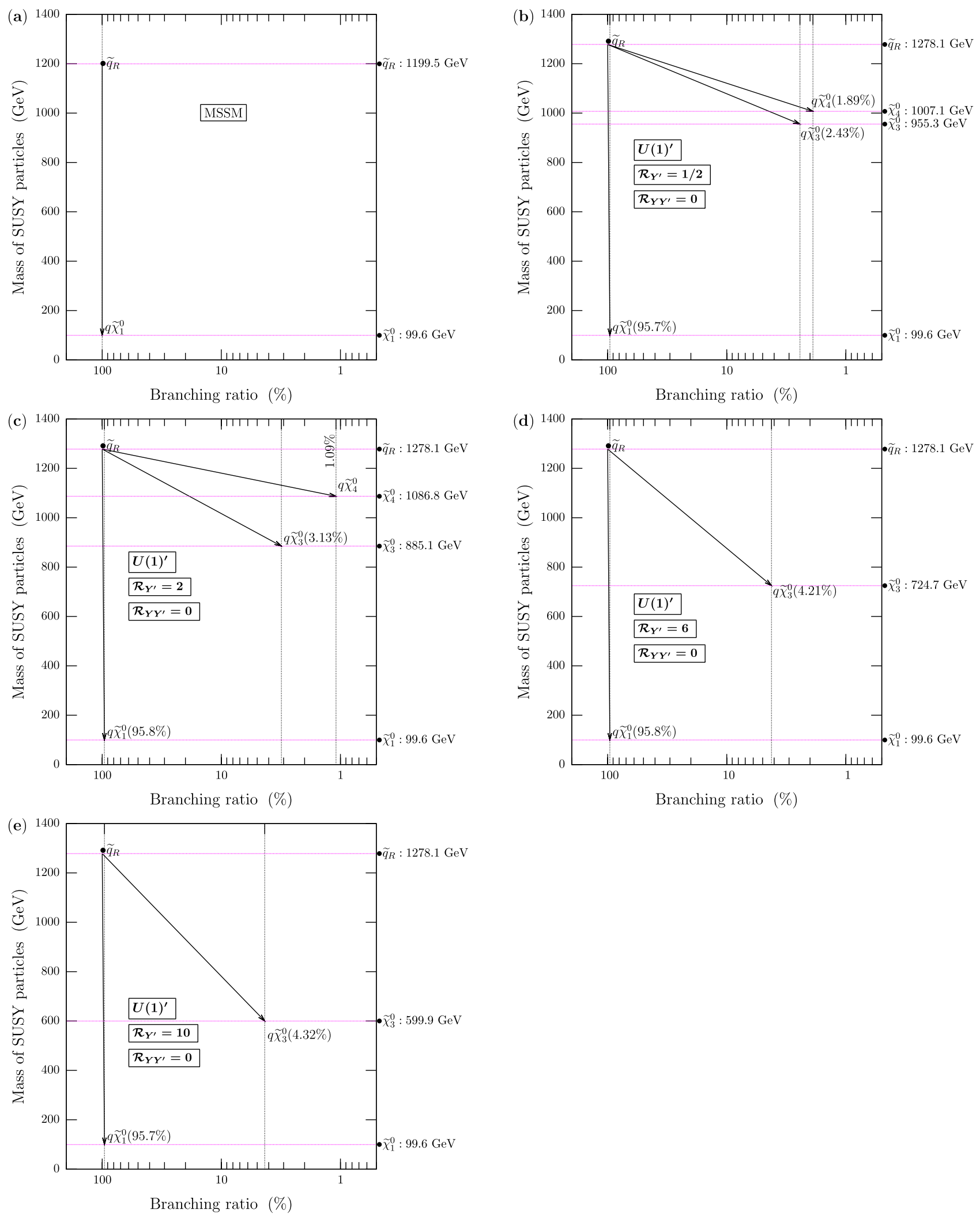

FIG. 1 (color online). The branching fractions $(\%)$ of right-handed squarks $\tilde{q}_{R}$ belonging to the first or second generation as a function of the neutralino and chargino masses. Shown are branching fractions exceeding $1 \%$ level. The panel (a) stands for the MSSM expectation while the rest correspond to the parameter set in (36), that is, the small mixing regime. The branching into $q \tilde{\chi}_{3}^{0}$ grows with decreasing $M_{\tilde{\chi}_{3}^{0}}$. 

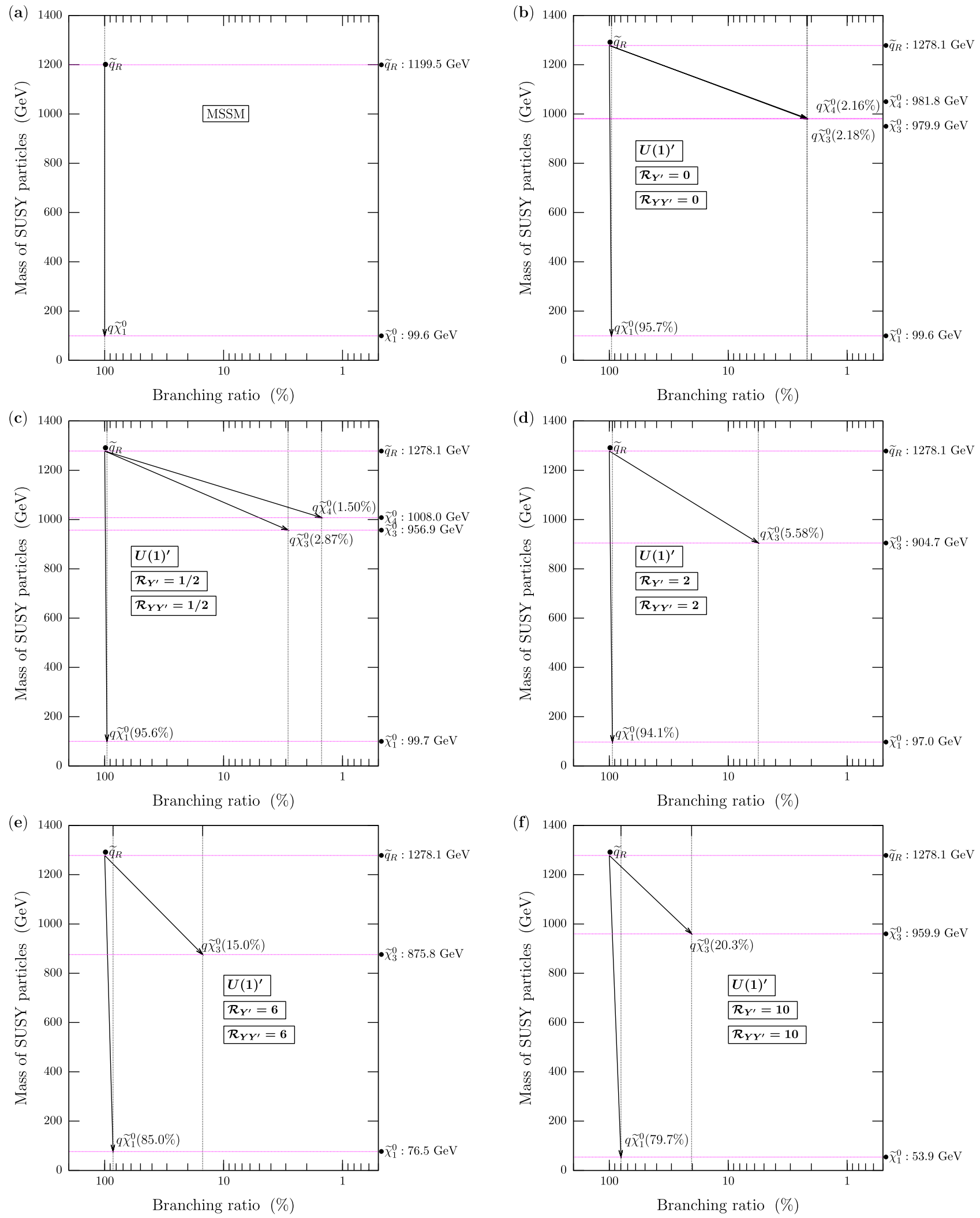

FIG. 2 (color online). The branching fractions (\%) of right-handed squarks $\tilde{q}_{R}$ belonging to the first or second generation as a function of the neutralino and chargino masses. Shown are branching fractions exceeding $1 \%$ level. The panel (a) stands for the MSSM expectation while the rest correspond to the parameter set in (37), that is, the medium mixing regime. The branching into $q \tilde{\chi}_{3}^{0}$ grows with decreasing $M_{\tilde{X}_{3}^{0}}$, and reaches the $20 \%$ level when $\mathcal{R}_{\tilde{Y}^{\prime}}=\mathcal{R}_{\tilde{Y} \tilde{Y}^{\prime}}=10$. 
ALI, DEMIR, FRANK, AND TURAN


PHYSICAL REVIEW D 79, 095001 (2009)
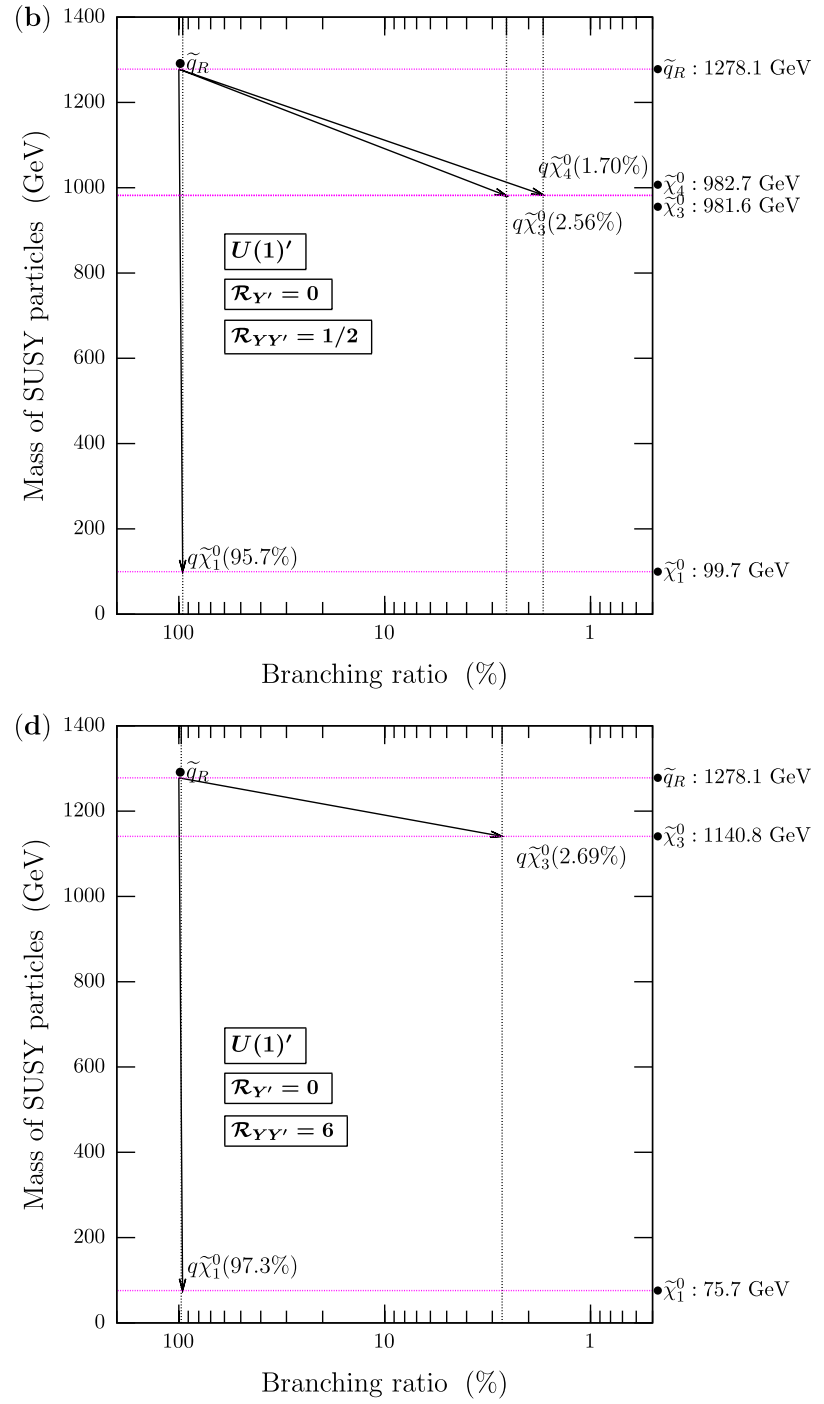

FIG. 3 (color online). The branching fractions (\%) of right-handed squarks $\tilde{q}_{R}$ belonging to the first or second generation as a function of the neutralino and chargino masses. Shown are branching fractions exceeding $1 \%$ level. The panel (a) stands for the MSSM expectation while the rest correspond to the parameter set in (38), that is, the large mixing regime. The branching into $q \tilde{\chi}_{3}^{0}$ decreases with increasing $M_{\tilde{\chi}_{3}^{0}}$, and is kinematically blocked when $\mathcal{R}_{\tilde{Y} \tilde{Y}^{\prime}}=10$. At this extreme, the branching of the squark is indistinguishable from the MSSM case. 

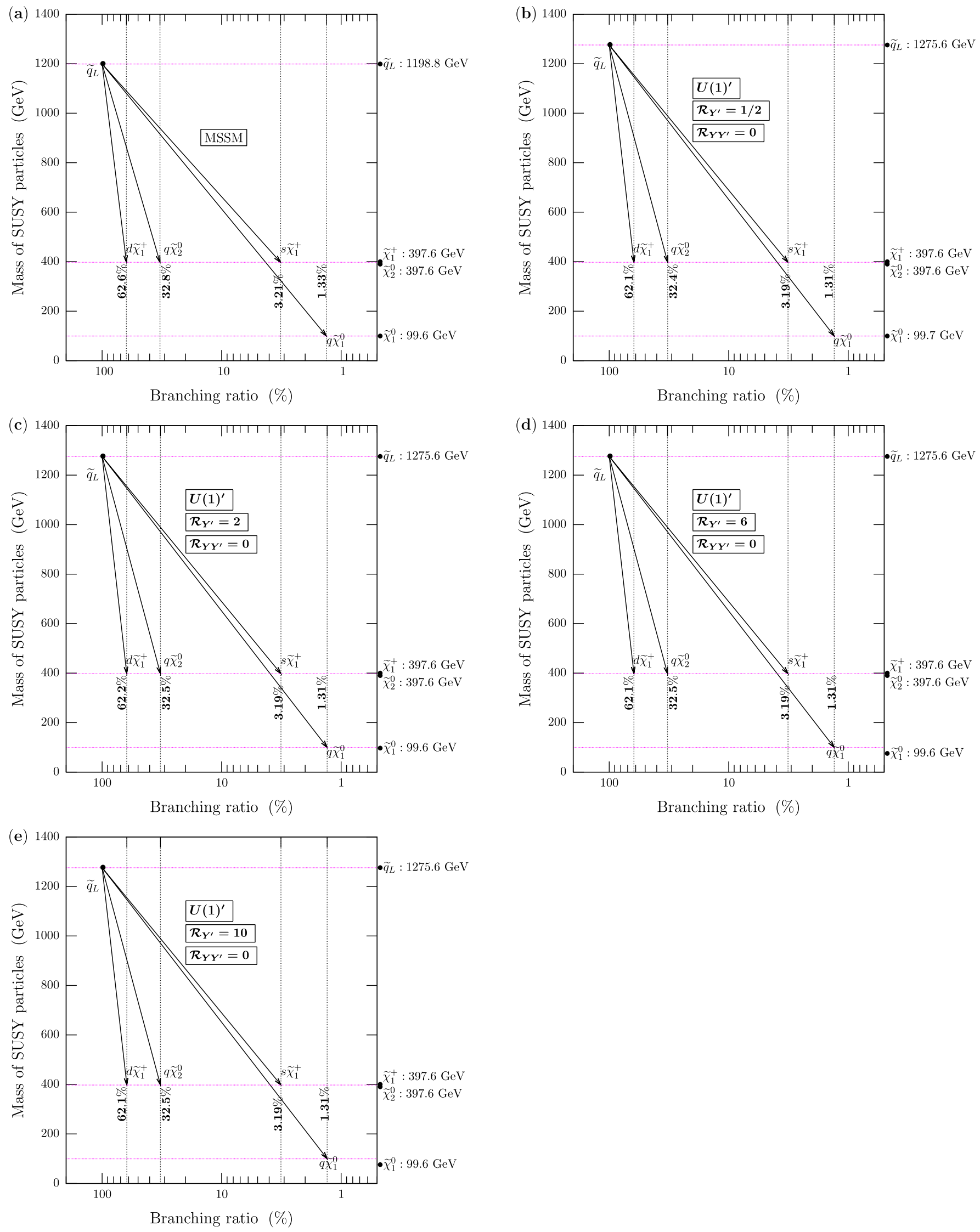

FIG. 4 (color online). The same as in Fig. 1 but for $\tilde{q}_{L}$. 

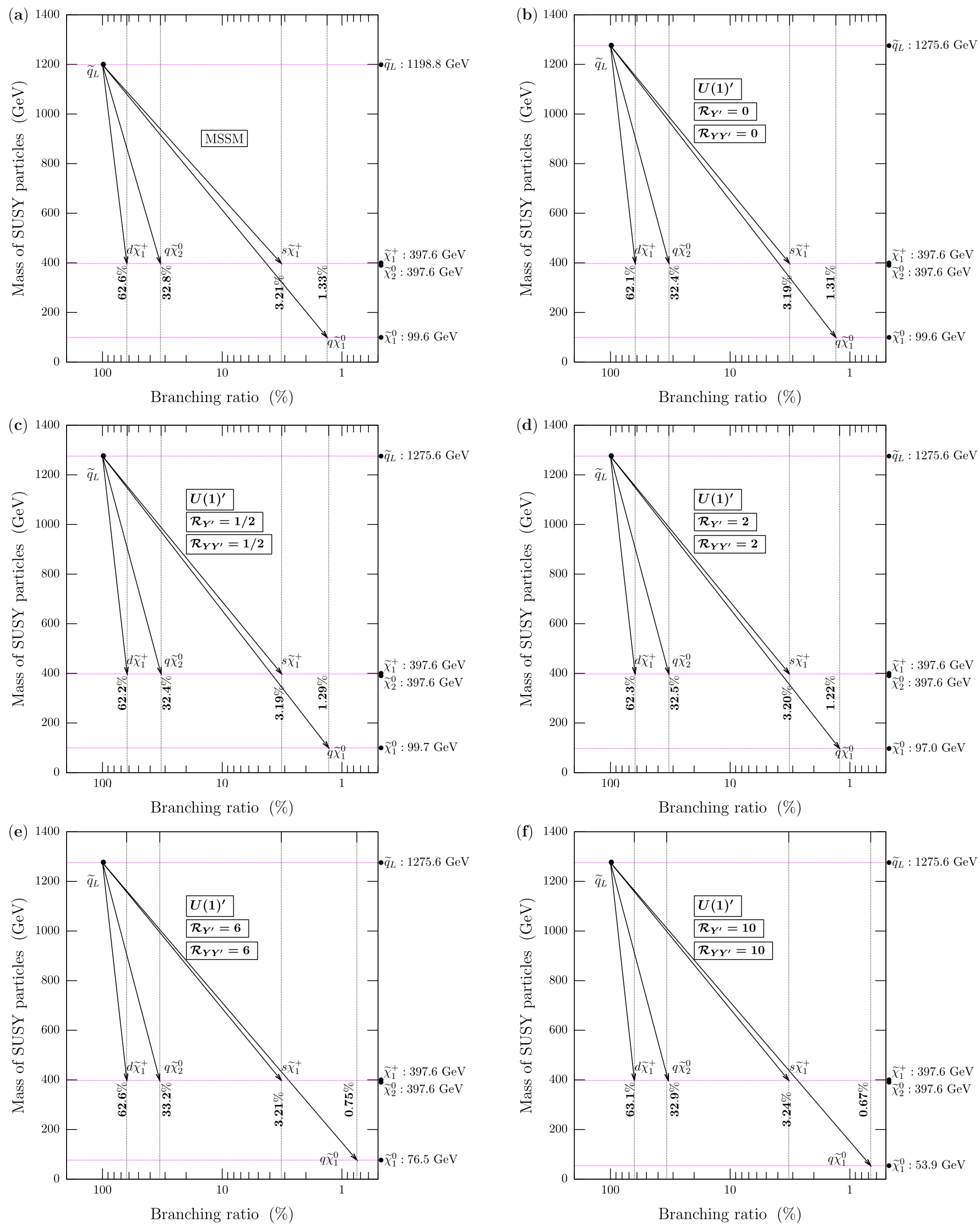

FIG. 5 (color online). The same as in Fig. 2 but for $\tilde{q}_{L}$. 

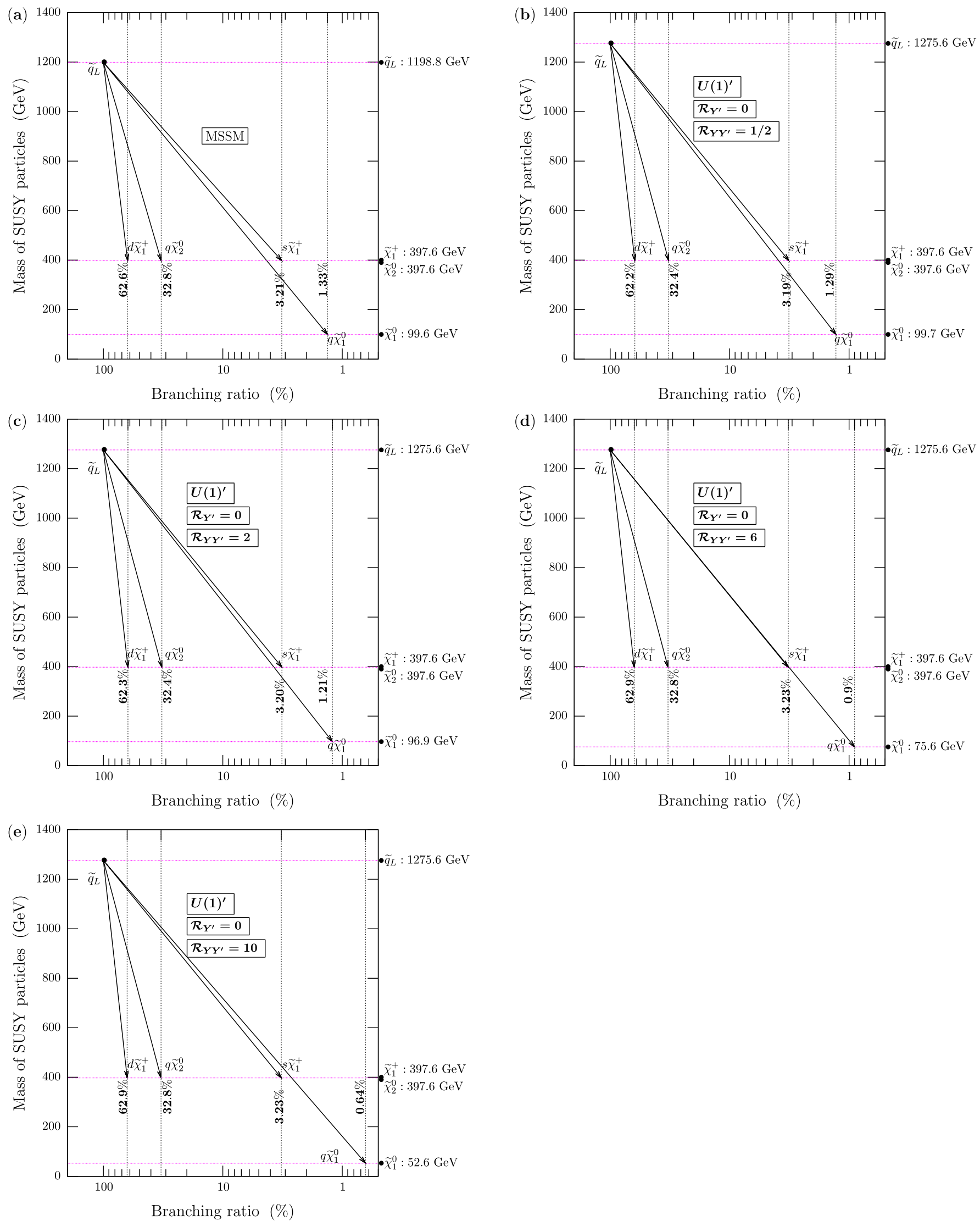

FIG. 6 (color online). The same as in Fig. 3 but for $\tilde{q}_{L}$. 
TABLE V. The basic LHC signals simulated with Monte Carlo event generators. Here $\ell=e$ or $\mu$, and "jets" stand for any number of jets in the final state. Each signal receives contributions from one or more decay processes, the strengths of which change as one switches from the MSSM to the $U(1)^{\prime}$ model. The candidate processes listed here involve only $N_{\text {jets }}=2$; the signals started by gluinos, which cause more jets than $N_{\text {jets }}=2$, are not shown.

\begin{tabular}{lcc}
\hline \hline SIGNAL & FINAL STATE & CANDIDATE PROCESSES FOR $N_{\text {jets }}=2$ \\
\hline SIGNAL 1 & $0 \ell+$ jets $+\mathscr{E}_{T}$ & $p p \rightarrow\left(\tilde{q} \rightarrow q \tilde{\chi}_{1}^{0}\right)\left(\tilde{q} \rightarrow q \tilde{\chi}_{1}^{0}\right)$ \\
SIGNAL 2 & $1 \ell+$ jets $+\mathscr{E}_{T}$ & $p p \rightarrow\left(\tilde{q} \rightarrow q^{\prime} \ell \bar{\nu}_{\ell} \tilde{\chi}_{1}^{0}\right)\left(\tilde{q} \rightarrow q \tilde{\chi}_{1}^{0}\right)$ \\
SIGNAL 3A & $2 \ell+$ jets $+\not{E}_{T}$ & $p p \rightarrow\left(\tilde{q} \rightarrow q^{\prime} \ell \bar{\nu}_{\ell} \tilde{\chi}_{1}^{0}\right)\left(\tilde{q} \rightarrow q^{\prime} \ell \bar{\nu}_{\ell} \tilde{\chi}_{1}^{0}\right)$ \\
SIGNAL 3B & & $p p \rightarrow\left(\tilde{q} \rightarrow q \ell^{+} \ell^{-} \tilde{\chi}_{1}^{0}\right)\left(\tilde{q} \rightarrow q \tilde{\chi}_{1}^{0}\right)$ \\
SIGNAL 4A & $3 \ell+$ jets $+\mathbb{E}_{T}$ & $p p \rightarrow\left(\tilde{q} \rightarrow q^{\prime} \ell \bar{\nu}_{\ell} \ell^{\prime+} \ell^{\prime-} \tilde{\chi}_{1}^{0}\right)\left(\tilde{q} \rightarrow q \tilde{\chi}_{1}^{0}\right)$ \\
SIGNAL 4B & & $p p \rightarrow\left(\tilde{q} \rightarrow q^{\prime} \ell \bar{\nu}_{\ell} \tilde{\chi}_{1}^{0}\right)\left(\tilde{q} \rightarrow q \ell^{\prime+} \ell^{\prime-} \tilde{\chi}_{1}^{0}\right)$ \\
\hline \hline
\end{tabular}

(ii) The transverse energy of the jets $E_{T}^{\text {jets }}$ with bin size $=$ $3 \mathrm{GeV}$,

(iii) The missing transverse energy $\mathbb{E}_{T}$ with bin size $=$ $20 \mathrm{GeV}$

(iv) The scalar sum of the transverse energies of the jets and leptons $E_{T}^{\text {sum }}$ with bin size $=40 \mathrm{GeV}$,

(v) The transverse momentum of the hardest lepton $p_{T}\left(\ell_{\text {hard }}\right)$ with bin size $=10 \mathrm{GeV}$,

(vi) The dilepton invariant mass $M_{\text {inv }}(\ell \ell)$ with bin size $=19 \mathrm{GeV}$.

Distributions with respect to these variables are expected to provide a global picture of the distinctive features of the events in Table $\mathrm{V}$ in regard to a comparative analysis of the MSSM and the $U(1)^{\prime}$ models.

The SIGNAL 1 in Table $\mathrm{V}$ is analyzed in Figs. 7-9. Similarly, SIGNAL 2 is analyzed in Figs. 10-12, SIGNAL $3 \mathrm{~A}$ in Figs. 13-15, SIGNAL 3B in Figs. 16-18, and finally SIGNAL 4B in Figs. 19-21. We discuss these plots in terms of their ability to discriminative between the
MSSM and $U(1)^{\prime}$ models. In these plots, we include contributions from all possible squark pair-production channels: $\tilde{q}_{R} \tilde{q}_{R}, \tilde{q}_{L} \tilde{q}_{L}$, and $\tilde{q}_{L} \tilde{q}_{R}$. In addition, we include the effects of the pair-production of the gluinos $\tilde{g} \tilde{g}$ as well as the associated production of the gluinos and squarks, $\tilde{g} \tilde{q}_{L, R}$. We combine contributions from all light quarks (the ones in the first and second generations) as jets in the final state without distinguishing quarks and antiquarks.

Figures 7-9 depict the number of purely hadronic events (SIGNAL 1 in Table V) as functions of the variables listed above. Figure 7 shows how the number of purely hadronic events vary with the number and transverse energy threshold of the jets. It is seen that the low-energy jets $E_{T}^{\text {jets }}>$ $20 \mathrm{GeV}$ exhibit a broad distribution over $N_{\text {jets }}=2$ (from the squark pair-production), $N_{\text {jets }}=3$ (from the gluinosquark associated production), $N_{\text {jets }}=4$ (from the gluino pair-production), and $N_{\text {jets }} \geq 5$ (from various multiple production and decay processes). As the transverse jet energy
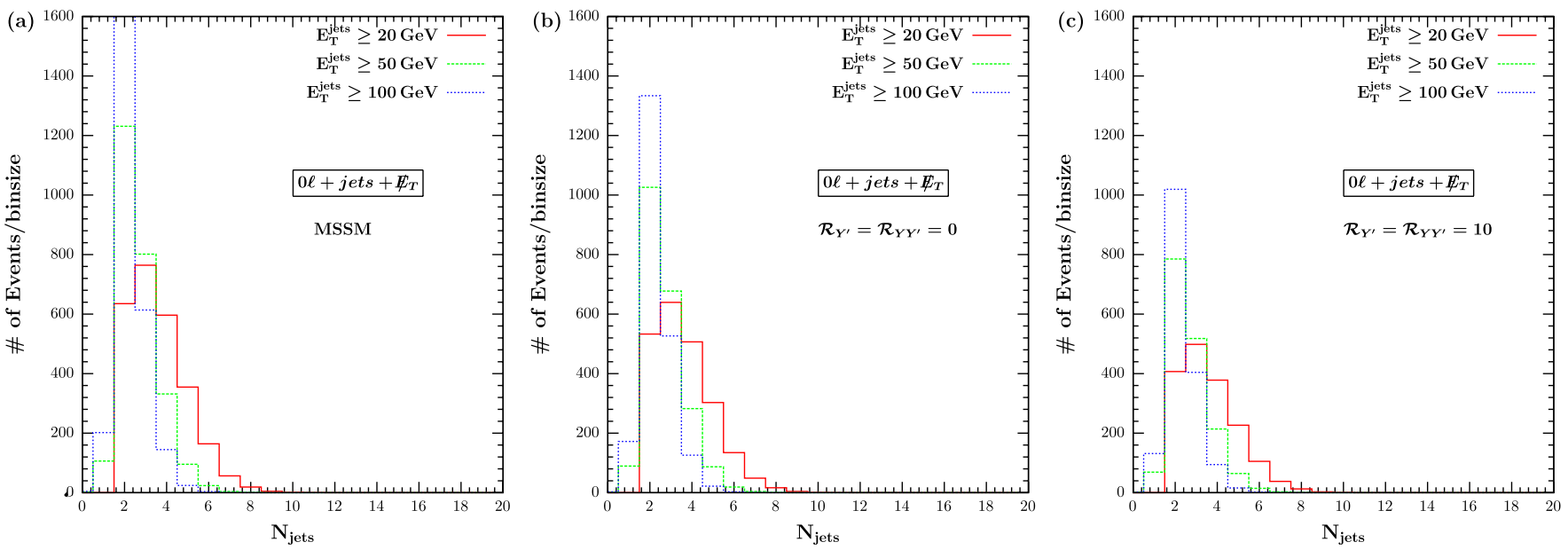

FIG. 7 (color online). The binwise (bin size $=1 \mathrm{GeV}$ ) distribution of the number of purely hadronic events (the events of the type SIGNAL 1 in Table V) with the number of jets $N_{\text {jets }}$ for different $E_{T}^{\text {jets }}$ ranges at an integrated luminosity of $\mathcal{L}=100 \mathrm{fb}^{-1}$ in the MSSM (panel (a)) and in the $U(1)^{\prime}$ model with $\left(\mathcal{R}_{Y^{\prime}}, \mathcal{R}_{Y Y^{\prime}}\right)=(0,0)$ (panel (b)) and $\left(\mathcal{R}_{Y^{\prime}}, \mathcal{R}_{Y Y^{\prime}}\right)=(10$, 10) (panel (c)). The number of hadronic events, in agreement with the discussions of Sec. III, are depleted in the $U(1)^{\prime}$ model compared to the MSSM. It is clear that the larger the transverse energy of the jets the closer the event is to dijet type. 

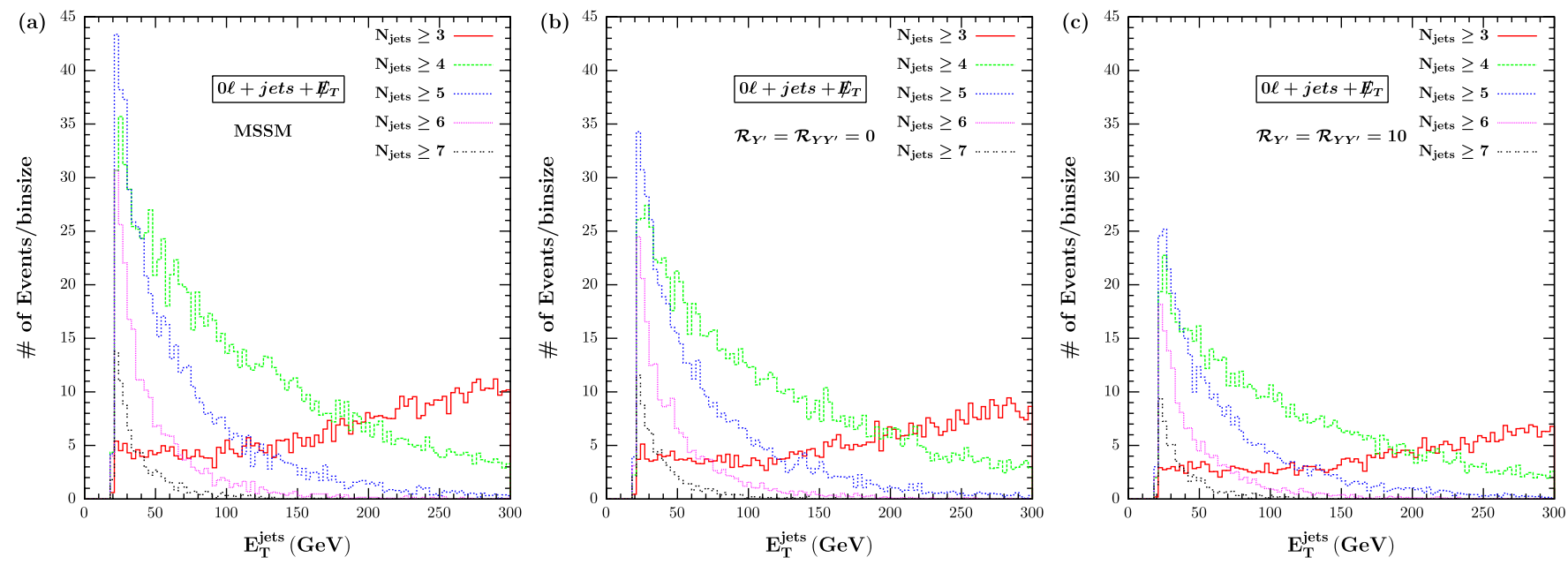

FIG. 8 (color online). The binwise (bin size $=3 \mathrm{GeV}$ ) distribution of the number of purely hadronic events (the events of the type SIGNAL 1 in Table V) with $E_{T}^{\text {jets }}$ for different $N_{\text {jets }}$ ranges at an integrated luminosity of $\mathcal{L}=100 \mathrm{fb}^{-1}$ in the MSSM (panel (a)) and in the $U(1)^{\prime}$ model with $\left(\mathcal{R}_{Y^{\prime}}, \mathcal{R}_{Y Y^{\prime}}\right)=(0,0)$ (panel (b)) and $\left(\mathcal{R}_{Y^{\prime}}, \mathcal{R}_{Y Y^{\prime}}\right)=(10,10)$ (panel (c)). The events with $N_{\text {jets }} \geq 4$ are soft (they are abundant only at low $E_{T}^{\text {jets }}$ ) and rare (they are few at large $E_{T}^{\text {jets }}$ ). The events with smaller numbers of jets are effective for a wide range of $E_{T}^{\text {jets }}$ values. In accord with Fig. 7, the purely hadronic events in the $U(1)^{\prime}$ model are fewer than in the MSSM, especially for the large $N_{\text {jets }}$ values.

increases, the distribution becomes less broad. In fact, for $E_{T}^{\text {jets }} \geq 100 \mathrm{GeV}$, the events are nearly pure dijet events induced by pair-production of squarks. The three panels, panels (a), (b) and (c), differ mainly by the overall change in the number of events as one switches from the MSSM to the $U(1)^{\prime}$ model. Indeed, purely hadronic events are depleted in number in the $U(1)^{\prime}$ model compared to the MSSM, and the depletion is strongest for $\left(\mathcal{R}_{\tilde{Y} \prime}, \mathcal{R}_{\tilde{Y} \tilde{Y}^{\prime}}\right)=$ $(10,10)$.

Figure 8 is complementary to Fig. 7, depicting the variation of the number of purely hadronic events
(SIGNAL 1 in Table V) with the jet transverse energy for different lower bounds on the number of jets. We see that the events with $N_{\text {jets }} \geq 4$ are soft (they dominate only at low $E_{T}^{\text {jets }}$ ) and rare (they rapidly decrease in number with increasing $\left.E_{T}^{\text {jets }}\right)$. The main distinction between the MSSM and the $U(1)^{\prime}$ models is the depletion of the number of events in the latter. The panel (a) of Fig. 9 depicts an important distribution: The variation of the purely hadronic events with the missing transverse energy $\mathscr{E}_{T}$. It is obvious that the number of events is maximal for the MSSM and decreases gradually in the $U(1)^{\prime}$ model as $\mathcal{R}_{\tilde{Y^{\prime}}}$ and/or $\mathcal{R}_{\tilde{Y} \tilde{Y}^{\prime}}$
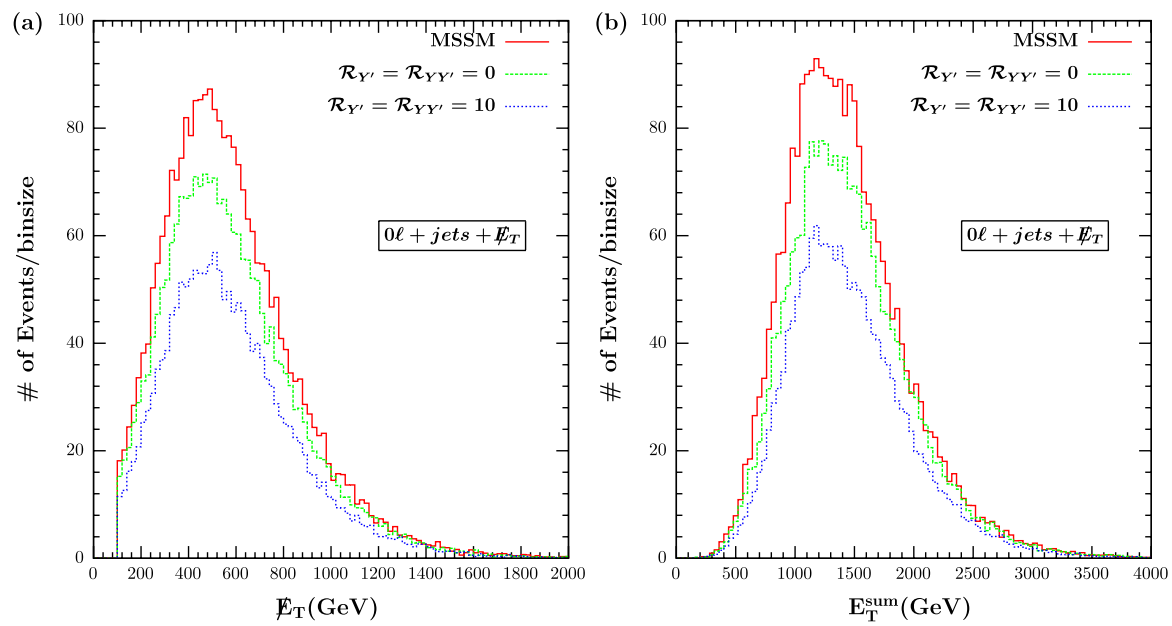

FIG. 9 (color online). The binwise distribution of the number of purely hadronic events (the events of the type SIGNAL 1 in Table V) with $\mathbb{E}_{T}$ (panel (a), bin size $=20 \mathrm{GeV}$ ) and $E_{T}^{\text {sum }}$ (panel (b), bin size $=40 \mathrm{GeV}$ ) at an integrated luminosity of $\mathcal{L}=100 \mathrm{fb}^{-1}$ in the MSSM and the $U(1)^{\prime}$ model. The central values of the distributions vary little from model to model. Nevertheless, the number of events are fewer in the $U(1)^{\prime}$ model than in the MSSM. This feature is in accordance with the discussion in Sec. III and with Figs. 7 and 8. 

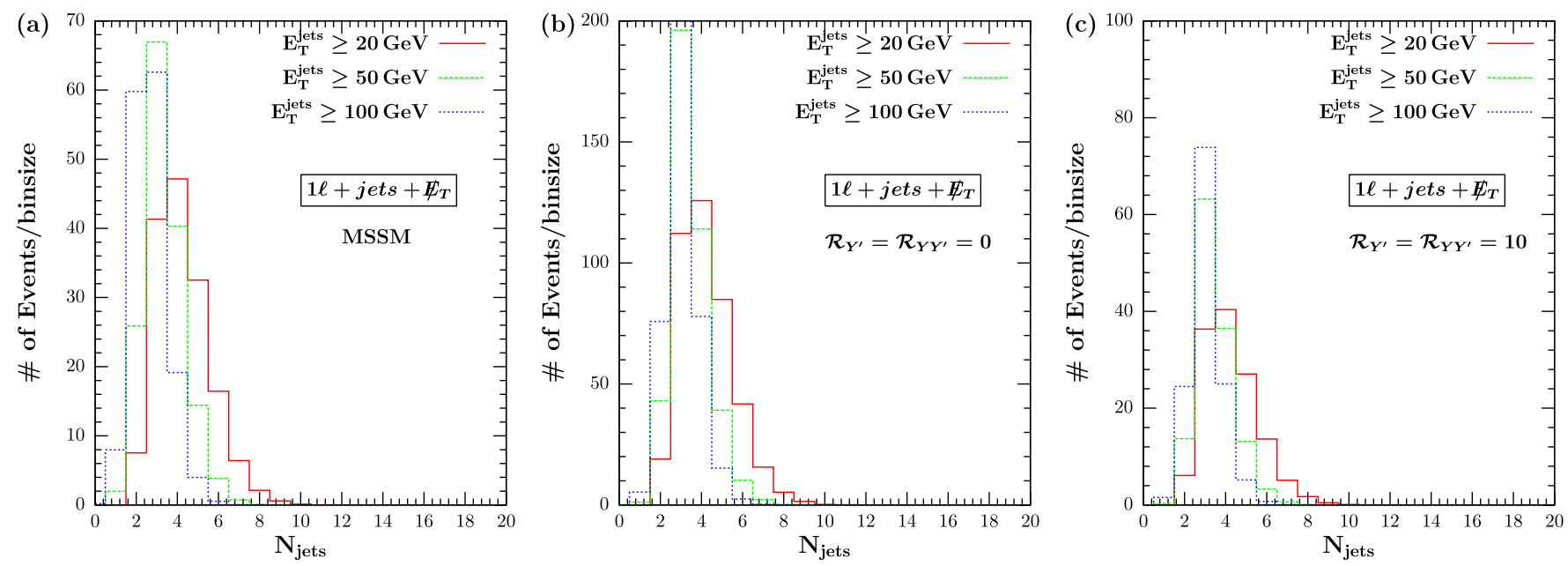

FIG. 10 (color online). The same as in Fig. 7 but for the single-lepton events (i.e., events of the type SIGNAL 2 in Table V).

increase. The distribution has a sharp edge at the LSP mass, and peaks around $500 \mathrm{GeV}$ with slight shifts depending on the details of the underlying model. The panel (b) of Fig. 9 shows the distribution as a function of the scalar sum of the transverse energies (missing transverse energy in panel (a)). Again, one notices the drop in the number of events as one switches from the MSSM to the $U(1)^{\prime}$ model. Clearly, the $E_{T}^{\text {sum }}$ value at which the distribution is maximized corresponds to the average squark/gluino masses. This distribution, traditionally, has been utilized to provide a shortcut to the scale of SUSY [16]. It is a sensitive variable to be searched for at the LHC.

Summarizing, the plots in Figs. 7-9 show that the purely hadronic events are more abundant in the MSSM than in the $U(1)^{\prime}$ model. All distributions are quite similar with fewer events for the $U(1)^{\prime}$ model case. These results confirm the discussions in Sec. III, and are consistent with the fact that the branching ratios $\mathcal{B}\left(\tilde{q}_{L, R} \rightarrow q \tilde{\chi}_{1}^{0}\right)$ in (24) are larger in the MSSM than in the $U(1)^{\prime}$ model.
An important feature to note is that the SIGNAL 1 is the most abundant among all the signals listed in Table $\mathrm{V}$ and studied in Figs. 10-21. This purely hadronic event, with no hard muons, can be constructed with good precision at the LHC with optimized jet algorithms. Measurement of the number of events for the given kinematic variables can facilitate the decision-making about the underlying model. We emphasize that the MSSM and the $U(1)^{\prime}$ models differ mainly by the number of events per bin size rather than by their distribution patterns.

Depicted in Figs. 10-12 are the distributions for the single-lepton events (SIGNAL 2 in Table V). The number and patterns of the events in the $U(1)^{\prime}$ model dominate (for $\mathcal{R}_{\tilde{Y}^{\prime}}=\mathcal{R}_{\tilde{Y} \tilde{Y}^{\prime}}=0$ ) or are comparable (for $\mathcal{R}_{\tilde{Y}^{\prime}}=\mathcal{R}_{\tilde{Y} \tilde{Y}^{\prime}}=$ $10)$ to the one in the MSSM. This behavioral change can be ascribed to the $\tilde{Z}^{\prime}$ mediation, as discussed in Sec. III. Figure 12, compared to Fig. 9, has one added feature, namely, the variation of the numbers of events with the transverse momentum of the emitted lepton. This plot, the
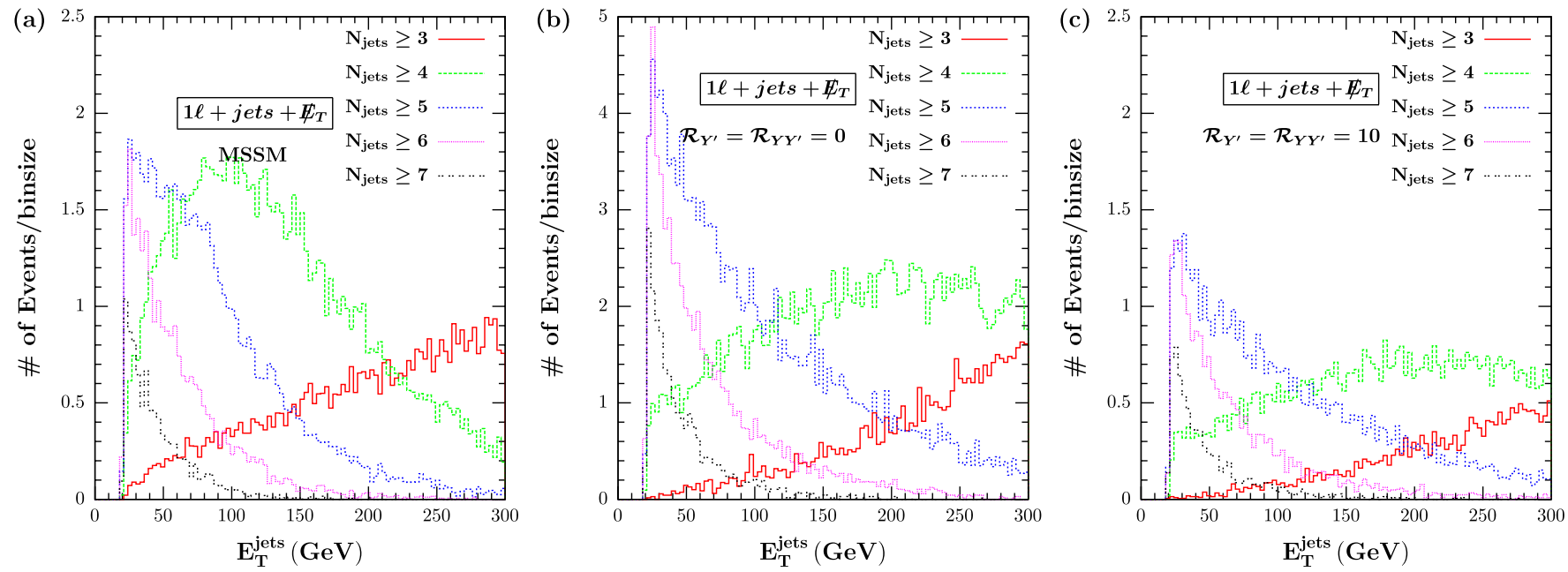

FIG. 11 (color online). The same as in Fig. 8 but for the single-lepton events (i.e., events of the type SIGNAL 2 in Table V). 



FIG. 12 (color online). The same as in Fig. 9 but for the single-lepton events (i.e., events of the type SIGNAL 2 in Table V). The panel (a) (bin size $=10 \mathrm{GeV}$ ) is new; it describes the distribution with respect to the transverse momentum of the emitted lepton.

panel (a) of Fig. 12, proves to be highly discriminative between the MSSM and the $U(1)^{\prime}$ model as the latter offers a much broader distribution extending to large transverse momenta values for the lepton.

In general, for the SIGNAL 2, the $\tilde{q}_{L} \tilde{q}_{R}$ pair-production (with or without the $\tilde{g}$ contribution) dominates all the others. There are no events from $\tilde{q}_{R} \tilde{q}_{R}$ since $\mathcal{B}\left(\tilde{q}_{R} \rightarrow\right.$ $\left.q \tilde{\chi}_{1}^{0}\right) \sim 10^{-6}$ in either model and the $\tilde{q}_{L} \tilde{q}_{L}$ contribution is much smaller than $\tilde{q}_{L} \tilde{q}_{R}$. This is again directly related to the fact that $\mathcal{B}\left(\tilde{q}_{L} \rightarrow q \tilde{\chi}_{1}^{0}\right) \ll \mathcal{B}\left(\tilde{q}_{R} \rightarrow q \tilde{\chi}_{1}^{0}\right)$. Hence, the most dominant signal proceeds through $p p \rightarrow \tilde{g} \tilde{g} \rightarrow$ $\left(q \tilde{q}_{L}\right)\left(q^{\prime} \tilde{q}_{R}\right) \rightarrow q q^{\prime}\left(\tilde{q}_{L} \rightarrow q^{\prime \prime} \tilde{\chi}_{1}^{ \pm}\right)\left(\tilde{q}_{R} \rightarrow q^{\prime \prime \prime} \tilde{\chi}_{1}^{0}\right) \rightarrow q q^{\prime} q^{\prime \prime} q^{\prime \prime \prime} \times$ $\left(\tilde{\chi}_{1}^{ \pm} \rightarrow \ell \tilde{\nu}_{\ell}\right) \tilde{\chi}_{1}^{0} \rightarrow\left(\tilde{\nu}_{\ell} \rightarrow \nu_{l} \tilde{\chi}_{1}^{0}\right)\left(\ell q q^{\prime} q^{\prime \prime} q^{\prime \prime \prime} \tilde{\chi}_{1}^{0}\right) \rightarrow \ell \nu_{l} q q^{\prime} q^{\prime \prime} \times$ $q^{\prime \prime \prime} \tilde{\chi}_{1}^{0} \tilde{\chi}_{1}^{0}$. This observation is confirmed by Figs. 10 and 11 where the event is seen to be a 4-jet event at high $E_{T}^{\mathrm{jet}}$. The hardness of the lepton (the only one for this signal) is mainly determined by the mass difference $m_{\tilde{\chi}_{1}^{ \pm}}-m_{\tilde{\nu}_{\ell}}$ which is about $50 \mathrm{GeV}$ in the MSSM but around $340 \mathrm{GeV}$ in the $U(1)^{\prime}$ model. Therefore, larger lepton $p_{T}$ cuts would help distinguish the $U(1)^{\prime}$ model from the MSSM. As mentioned before, both $E_{T}^{\text {sum }}$ and $\mathscr{E}_{T}$ distributions are dominated by the $U(1)^{\prime}$ model events (most visibly in the $\left(\mathcal{R}_{Y^{\prime}}, \mathcal{R}_{Y Y^{\prime}}\right)=(0,0)$ case $)$.

In Figs. 13-18, we show the number of events containing two charged leptons in the final state (SIGNAL 3A and SIGNAL 3B in Table V). The distributions of these dilepton events are expected to reveal further distinctive features of the two models. By contrasting the distributions in Figs. 13 and 14 with those in Figs. 16 and 17, one finds that the SIGNAL 3A is dominantly a 4-jet event at high $E_{T}^{\mathrm{jets}}$ whereas the SIGNAL 3B involves both 3-jet and 4-jet topologies depending on $E_{T}^{\mathrm{jets}}$ range. It is convenient to start the analysis with the dilepton signal of SIGNAL 3A type. In this event, each charged lepton originates from a differ-
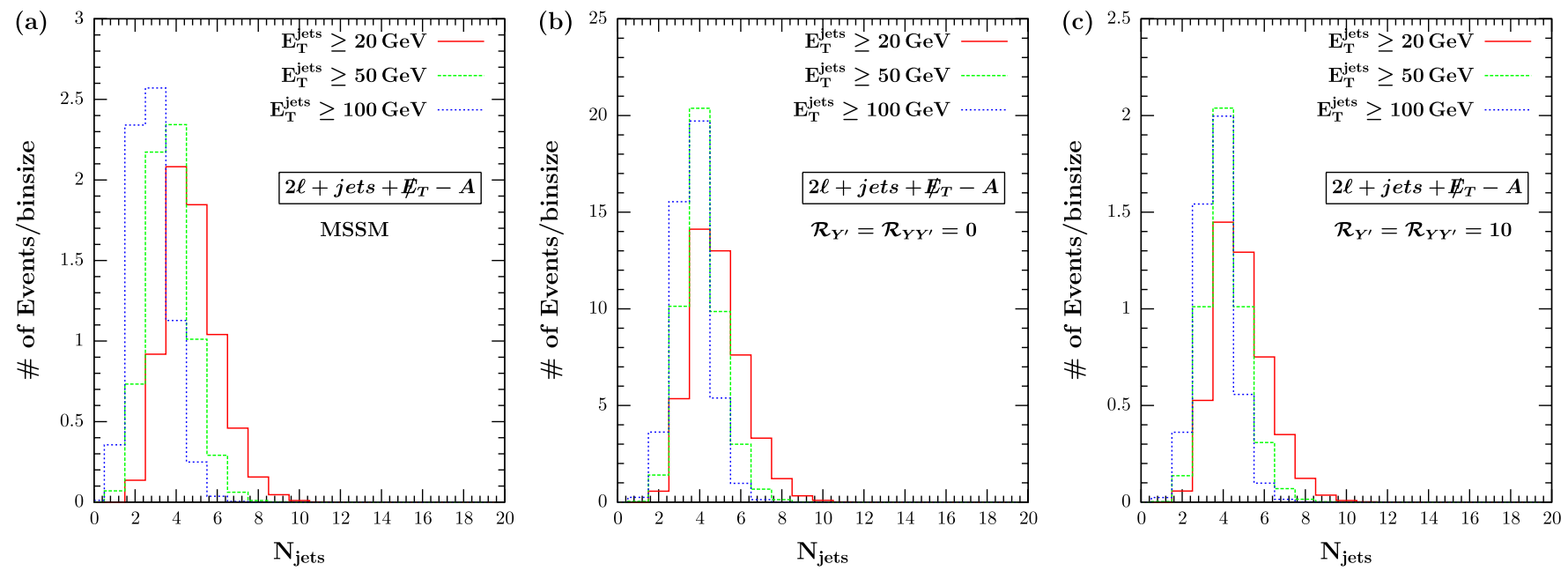

FIG. 13 (color online). The same as in Fig. 7 but for the dilepton events (i.e., events of the type SIGNAL 3A in Table V). 
ALI, DEMIR, FRANK, AND TURAN

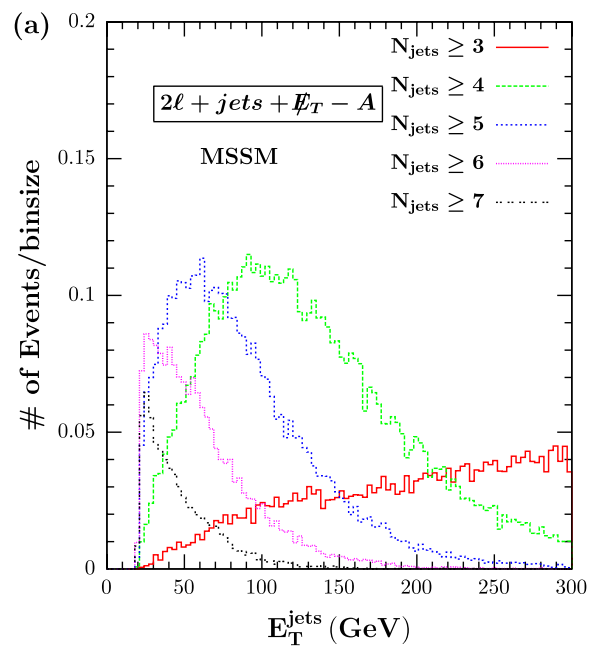



PHYSICAL REVIEW D 79, 095001 (2009)

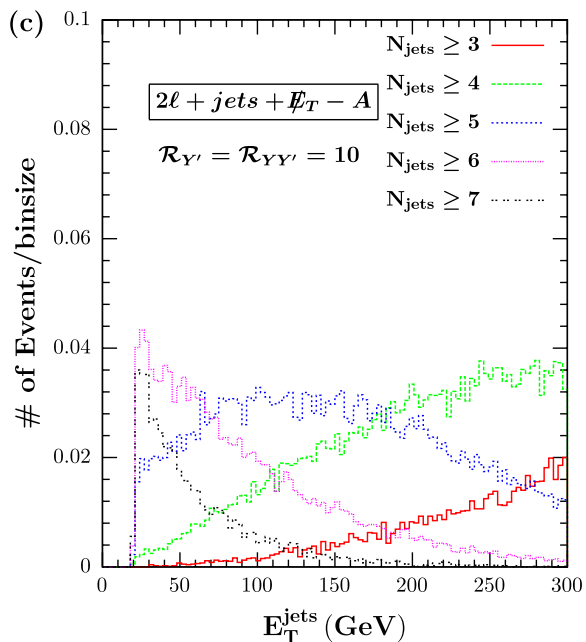

FIG. 14 (color online). The same as in Fig. 8 but for the dilepton events (i.e., events of the type SIGNAL 3A in Table V).
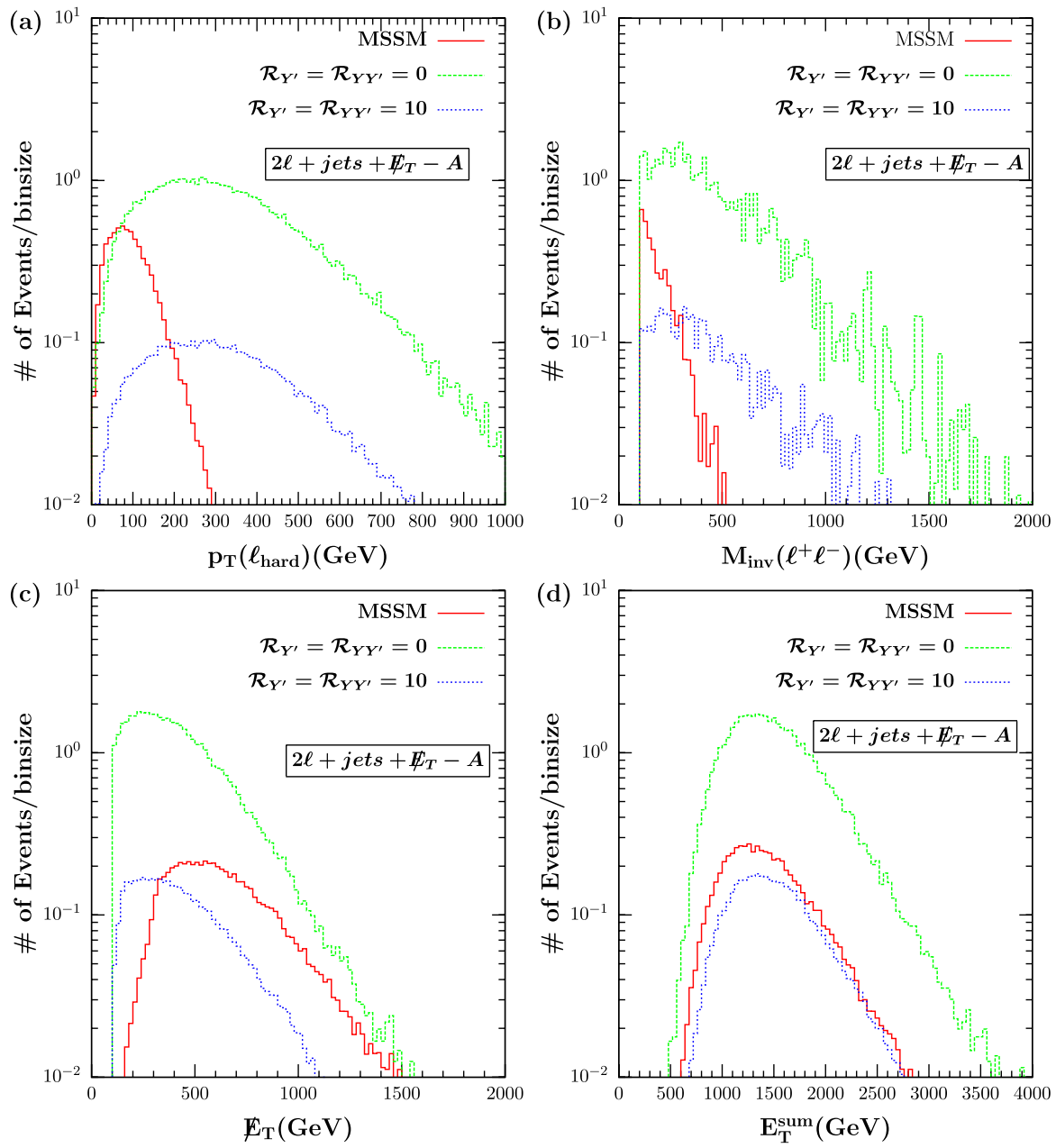

FIG. 15 (color online). The same as in Fig. 12 but for the dilepton events (i.e., events of the type SIGNAL 3A in Table V). The new features compared to those in Fig. 12 are as follows: Panel (a) describes the distribution with respect to the transverse momentum of the hardest lepton, $p_{T}\left(\ell_{\text {hard }}\right.$ ). Panel (b) (bin size $=19 \mathrm{GeV}$ ) is new; it describes the distribution with respect to the invariant mass of the two emitted leptons, $M_{\text {inv }}\left(\ell^{+} \ell^{-}\right)$. 

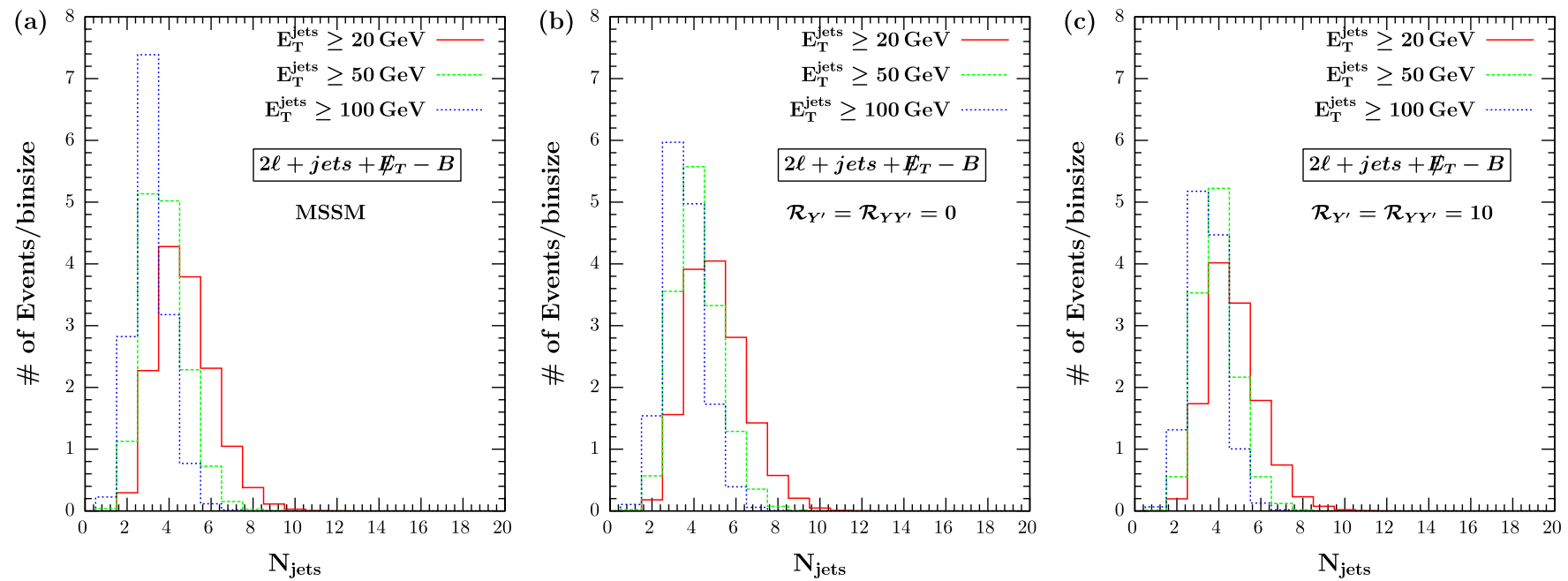

FIG. 16 (color online). The same as in Fig. 7 but for the dilepton events (i.e., events of the type SIGNAL 3B in Table V).

ent decay branch (started by squark or gluino). The $U(1)^{\prime}$ signal again dominates for $\left(\mathcal{R}_{Y^{\prime}}, \mathcal{R}_{Y Y^{\prime}}\right)=(0,0)$ and remains comparable to the MSSM case for $\left(\mathcal{R}_{Y^{\prime}}, \mathcal{R}_{Y Y^{\prime}}\right)=$ $(10,10)$. Unlike the SIGNAL 2 above, this process is dominated by the $\tilde{q}_{L} \tilde{q}_{L}$ contribution since both squarks need to decay into a chargino. Compared to Fig. 12, we have one additional plot, the panel (b) of Fig. 15, showing the number of events against the dilepton invariant mass $M_{\text {inv }}\left(\ell^{+} \ell^{-}\right)$. This distribution does not reveal a sharp edge since the leptons originate from different branches [24]. As in Fig. 12, the transverse momentum of the hardest of the two leptons emitted $p_{T}\left(\ell_{\text {hard }}\right)$ is capable of distinguishing the two models for large lepton $p_{T}$ cuts.

Compared to the SIGNAL 3A, the $p_{T}\left(\ell_{\text {hard }}\right)$ distribution hardly changes as one switches from the MSSM to the $U(1)^{\prime}$ model, especially at large $p_{T}$. This feature continues to hold for other distributions in Fig. 18, except for the dilepton invariant mass distribution. The reason for the discriminative nature of the $M_{\text {inv }}\left(\ell^{+} \ell^{-}\right)$distribution is that the two leptons originate from the same decay branch and obtain different distribution tails for different processes. The results are explicated in panel (b) of Fig. 18.

Looking closely, the $\tilde{q}_{R} \tilde{q}_{R}$ production-and-decay is a completely new contribution to this signal in $U(1)^{\prime}$, and the two models would give drastically different results if other contributions were ignored. This expectation, which follows from the discussions in Sec. III, is best examined by explicating the contributions of the individual squarks/ gluinos. We do this in panel (c) of Fig. 18 wherein the $\tilde{q}_{L}$ and $\tilde{q}_{R}$ contributions are explicated for the $\not{E}_{T}$ distribution. The entire signal is dominated by the $\tilde{q}_{L} \tilde{q}_{R}$ productionand-decay where $\tilde{q}_{R}$ decays to $q \tilde{\chi}_{1}^{0}$. Once we sum these subprocesses, the missing energy distribution in $U(1)^{\prime}$ is either almost the same or a little bit suppressed compared to the MSSM depending on the $\left(\mathcal{R}_{Y^{\prime}}, \mathcal{R}_{Y Y^{\prime}}\right)$ parameters.


FIG. 17 (color online). The same as in Fig. 8 but for the dilepton events (i.e., events of the type SIGNAL 3B in Table V). 

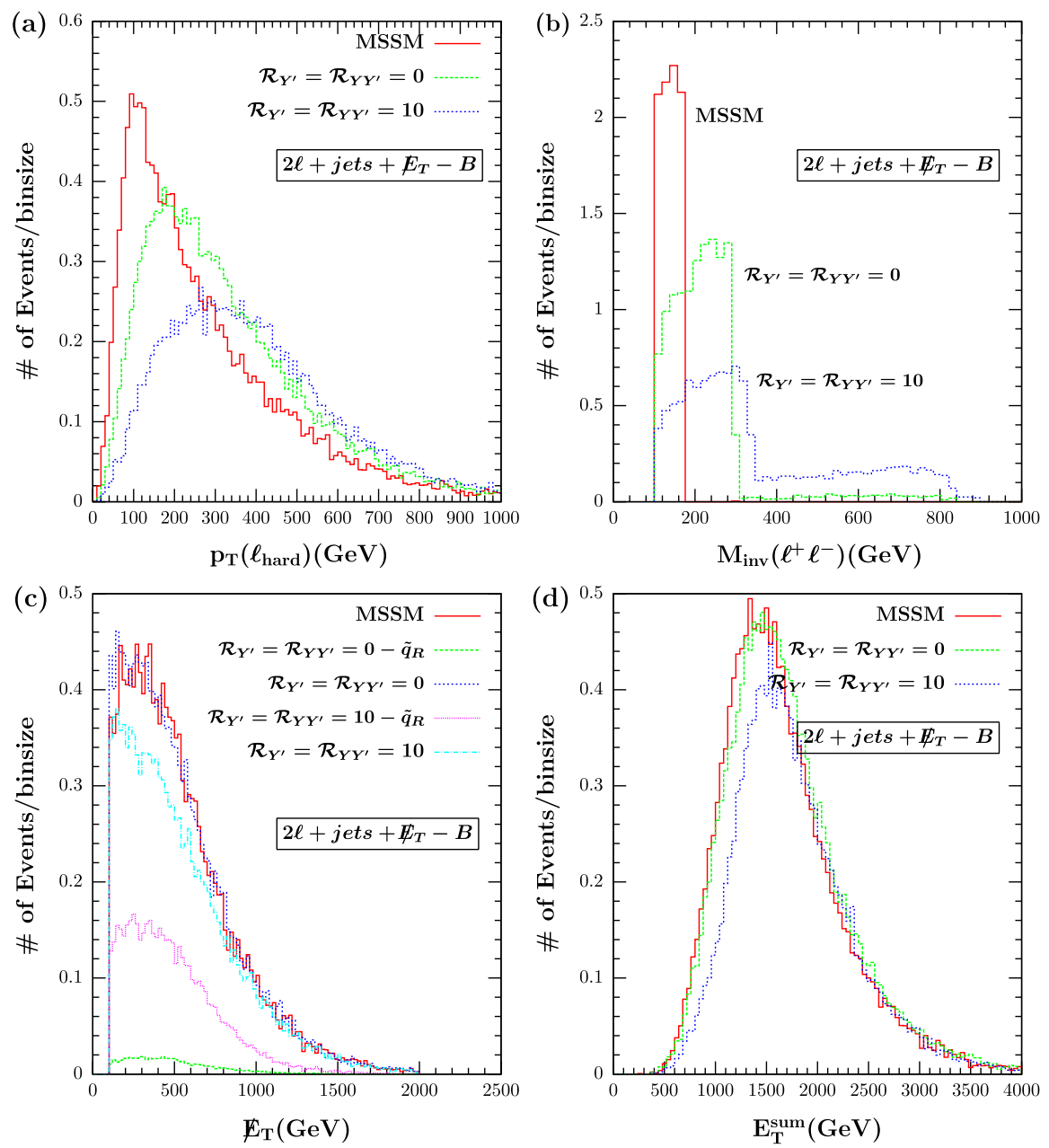

FIG. 18 (color online). The same as in Fig. 15 but for the dilepton events (i.e., events of the type SIGNAL 3B in Table V).

Depicted in Figs. 19-21 are the distributions of the trilepton event (SIGNAL 4B in Table V). Clearly, two oppositely charged leptons arise from one decay branch and the third one from the other branch. As shown in the figures, the two models can be distinguished via the number of events and their distributions. To emphasize the
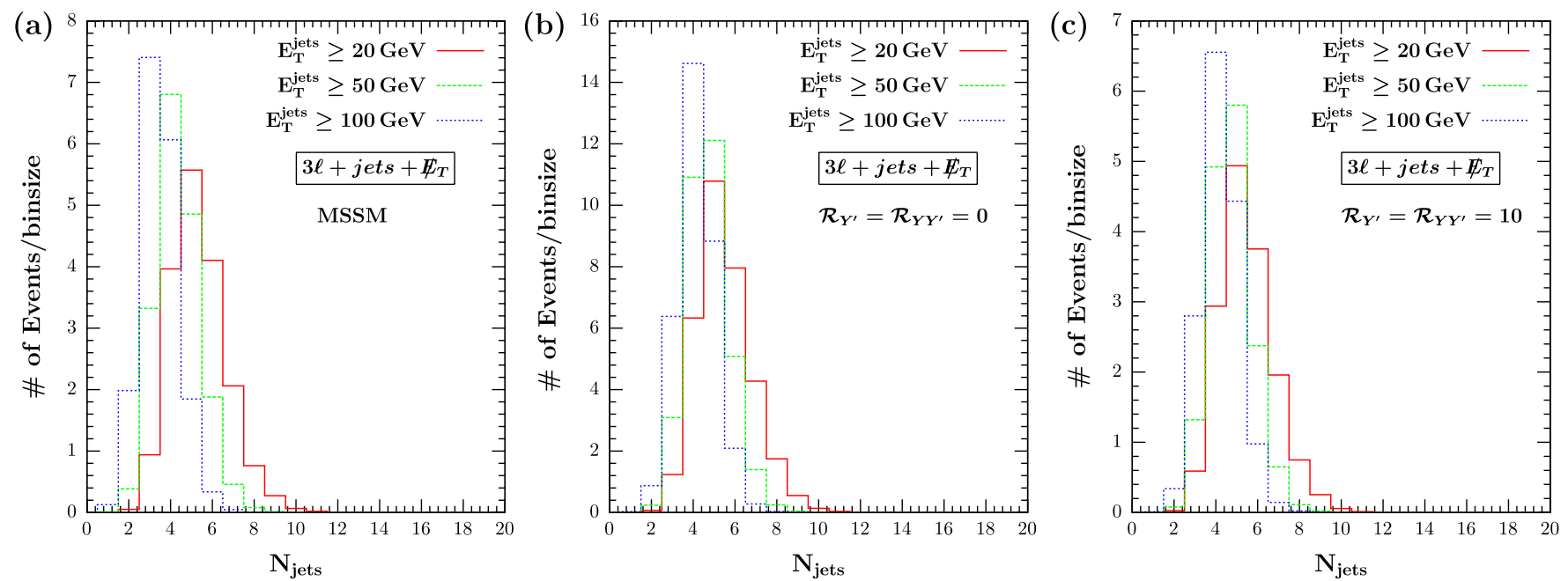

FIG. 19 (color online). The same as in Fig. 7 but for the trilepton events (i.e., events of the type SIGNAL 4B in Table V). 

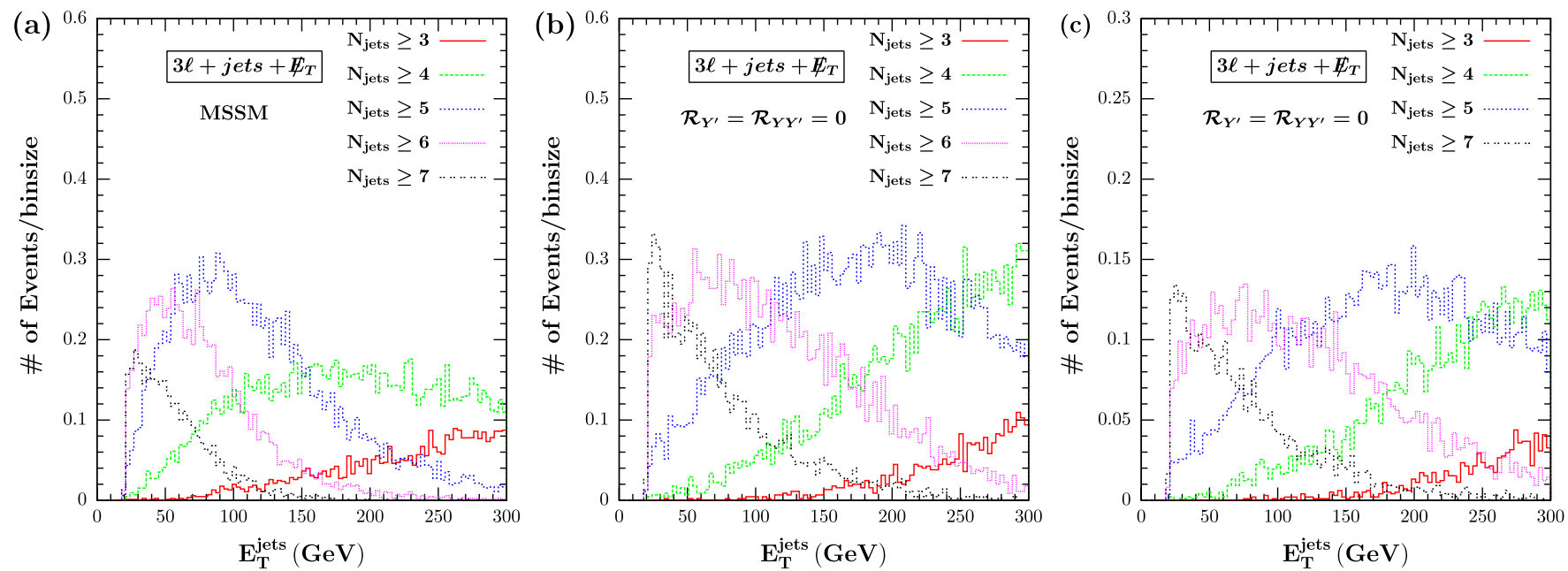

FIG. 20 (color online). The same as in Fig. 8 but for the trilepton events (i.e., events of the type SIGNAL 4B in Table V).
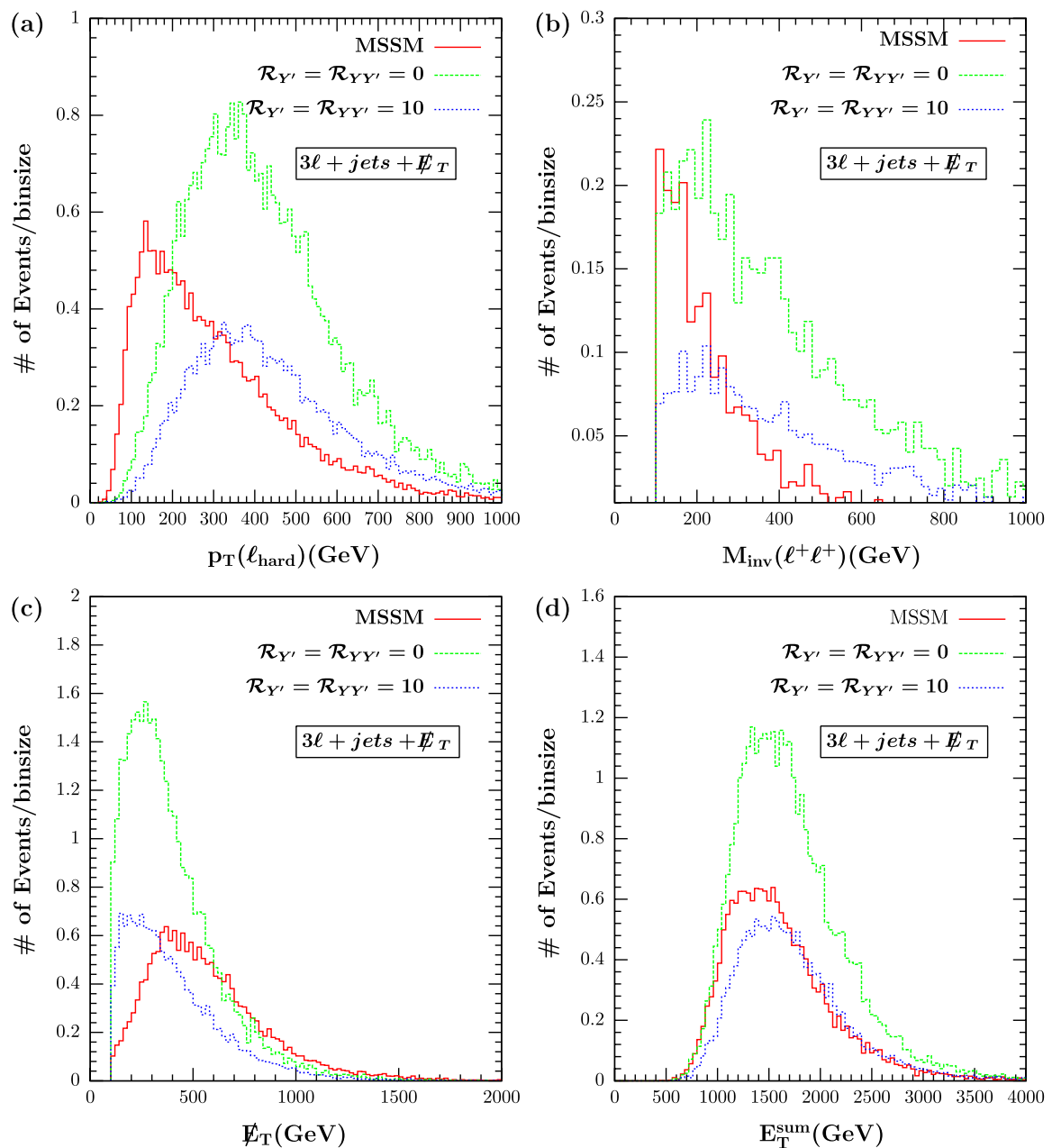

FIG. 21 (color online). The same as in Fig. 15 but for the trilepton events (i.e., events of the type SIGNAL 4B in Table V). The panel (b) is different than those in Figs. 15 and 18 in that it describes the distribution with respect to the invariant mass of the two samecharge leptons, $M_{\text {inv }}\left(\ell^{+} \ell^{+}\right)$. 
trilepton nature of the event, we plot in panel (b) of Fig. 21 the invariant mass of the two same-charge leptons which originate from the different branches (as in SIGNAL 3A).

Examining these features in depth for the SIGNAL 4B, even though the $\tilde{q}_{L} \tilde{q}_{L}$ contributions dominate in both the MSSM and the $U(1)^{\prime}$ model, a new effect shows up. The MSSM distributions receive contributions from the $\tilde{q}_{R} \tilde{q}_{R}$ production, but not the ones in the $U(1)^{\prime}$ model. The reason is that this signal requires one squark to decay into $q \tilde{\chi}_{2}^{0}$ and the other one into $q^{\prime} \tilde{\chi}_{1}^{ \pm}$. For $\tilde{q}_{R}$ in the MSSM, the branching fractions into $\tilde{\chi}_{2}^{0}$ and $\tilde{\chi}_{1}^{ \pm}$are small but comparable to each other, and they are the second largest branching ratios after the $q \tilde{\chi}_{1}^{0}$ mode. However, in the $U(1)^{\prime}, \tilde{q}_{R}$ possesses new neutral decay modes into $q \tilde{\chi}_{3}^{0}$ and $q \tilde{\chi}_{4}^{0}$, the branchings of which are of the order of $10^{-2}$. This suppresses the $q \tilde{\chi}_{1}^{ \pm}$ channel much further. We do not see these effects in the plots since the $\tilde{q}_{L} \tilde{q}_{L}$ decay mode dominates over the others.

The numerical studies of the branching fractions and event distributions convincingly prove that the MSSM and the $U(1)^{\prime}$ model can be discriminated at the LHC experiments. The purely hadronic events, classified as the SIGNAL 1 in Table V, turn out to be more abundant than the leptonic ones roughly by an order of magnitude. The analysis for confronting various distributions in the two models has been based on basic cuts. In analyzing the experimental data, certain signals, like the SIGNAL 3B, may require more detailed optimization cuts beyond the basic ones to enhance the $U(1)^{\prime}$ signal compared to the MSSM. Nevertheless, on general grounds, the two models behave differently in various kinematic observables, and measurements of events with different leptonic contents qualify to be a viable tool to disentangle the effects of the gauge-extended models from the bulk of data.

\section{CONCLUSION}

Once the LHC becomes fully functional, one of its most important tasks would be to discover physics beyond the standard model, and, in particular, to look for signals of supersymmetry, the most extensively studied scenario as such.

From previous studies it is well known that the signature of supersymmetry at the LHC would be fairly straightforward. One expects large excesses of events over the ones in the standard model with a number of characteristic signatures: for example, events with one or more isolated leptons, an excess of trilepton events, a pattern of missing $E_{T}$ plus jets and a characteristic $l^{+} l^{-}$invariant mass distribution.

What is not well studied is how would one be able to distinguish among different, realistic models of supersymmetry. Whereas many studies of the MSSM and mSUGRA models exist, fewer studies are available for the extended models. In this work, we have studied in depth the MSSM augmented by an extra $U(1)$ gauge symmetry, the $U(1)^{\prime}$ model. This model, devised to solve the supersymmetric $\mu$ problem, is further justified as a $\mathrm{TeV}$ scale remnant of the supersymmetric GUTs or string models. In an attempt to keep the model as generic as possible, we have fixed some of the model parameters (inspired by the supersymmetric $E_{6}$ GUT), restricted some parameters from the available experimental bounds, and varied the rest freely in some reasonable ranges. In Secs. II and III, we described the $U(1)^{\prime}$ model and the possible search strategies at hadron colliders. As an immediate consequence of the supersymmetric setup, we emphasized that the collider signatures of the model can be searched for by either considering the bosonic fields or the fermionic fields. The former has been under both phenomenological and experimental study, so we focused here on the effects of the fermionic fields with regard to their potential to reveal possible gauge extensions. As we expect that the squarks and gluinos will be abundantly produced at the LHC, we look for the $U(1)^{\prime}$ effects in their decays. As discussed in Sec. III and simulated in Sec. IV, we arrived at novel features in the generic LHC events which reveal the effects of gauge extension. Combined with the possible $Z^{\prime}$ discovery in the Drell-Yan process, the analysis and results of this work illustrate other discernible effects of a $U(1)^{\prime}$ extension.

The analysis reported here includes inherently some model and parameter-set dependence. Nevertheless, it predicts some clear distinguishing features of the $U(1)^{\prime}$ model from the MSSM. In particular, in this model, the righthanded squarks can decay through an extra neutral gaugino (in addition to the LSP) leading to an enhanced signal in the events containing at least one lepton. The difference between this model and the MSSM becomes also visible in the invariant mass distribution of the $\ell^{+} \ell^{-}$pair, and in the missing $E_{T}$ distribution. In spite of these promising observables, a more general analysis involving a fine-grained scan of a wider set of parameters (and not just the $U(1)^{\prime}$ gaugino mass and its mixing with the hypercharge gaugino, as employed in the present work), can reveal further properties that can be of interest at the LHC.

We summarize main findings of the simulation studies detailed in Sec. IV for the signals listed in Table V which have the generic form as $m \ell+n$ jets $+\not E_{T}$. The number of jets $n$ has to be at least two but could be bigger depending on the detailed composition in the production and/or in the cascade decays. We consider events with up to $m=3$ leptons, and arrive at the following features (in comparison to the MSSM):

(i) The SIGNAL 1 (no-lepton event) of Table V consisting of purely hadronic events. As expected, the number of events are fewer in the $U(1)^{\prime}$ model than in the MSSM. Various distributions such as the jet multiplicities, transverse energy of jets, missing transverse energy as well as the scalar sum of transverse energies are considered. The distributions for the two models are similar in topology with fewer 
signal events surviving for the $U(1)^{\prime}$ model, after applying the primary selection cuts. The number of signal events at the peak of the distributions is in the range of 10 to 100 , but none of the distributions is good enough to disentangle the $U(1)^{\prime}$ effects unless some secondary selection cuts are imposed.

(ii) For the SIGNAL 2 (one-lepton event) with one lepton in the final state, the $U(1)^{\prime}$ effects start becoming distinguishable not only in the number of events but also in the event topology. In particular, the $p_{T}$ distribution of the hardest lepton, as a new observable in addition to the ones discussed for SIGNAL 1, turns out to be very useful to distinguish the $U(1)^{\prime}$ effects (mainly in the high $p_{T}$-tail). The distribution is shown in panel (a) of Fig. 12. Unlike the $U(1)^{\prime}$ distributions, the MSSM distribution dies off rapidly since the available energy for the lepton is around $50 \mathrm{GeV}$ for the MSSM case but around $350 \mathrm{GeV}$ for the $U(1)^{\prime}$ case. The missing transverse energy and the scalar sum of the transverse energy distributions are also useful, and the $U(1)^{\prime}$ effects dominate over the MSSM ones for especially low $\mathcal{R}_{Y^{\prime}}$ and/or $\mathcal{R}_{Y Y^{\prime}}$ values. The number of signal events at the peak of the essential distributions is around 10, big enough for a discovery.

(iii) The SIGNALS 3A and 3B (two-lepton events) involve a lepton pair where both the leptons come from different branches for SIGNALS 3A, and from the same branch for SIGNALS 3B. This is evident from the invariant mass distribution of the lepton pair, depicted in panel (b) of Figs. 15-18. While the distributions for the $p_{T}$ of the hardest lepton, the dilepton invariant mass, as well as the missing transverse energy and the scalar sum of the transverse energies prove useful to disentangle the $U(1)^{\prime}$ effects in the SIGNAL 3A case, only two of them are promising in the SIGNAL 3B case, as the MSSM and the $U(1)^{\prime}$ model lead to comparable contributions in the distributions of missing transverse energy and the scalar sum of transverse energies. Again, only few events at the peak of primary observables qualify to be signals, in each case.

(iv) For the SIGNALS 4A and 4B (three-lepton events), there are three leptons, all coming from the same branch for the SIGNALS 4A. Thus, the SIGNAL 4A events in our parameter set require $1 \rightarrow 4$ decays and are not considered any further. For the SIGNAL 4B events, however, we analyze, in addition to the others, the same-sign-same-flavor lepton pair invariant mass distribution (which is unique to the trilepton signal, in general). In all these distributions, the $U(1)^{\prime}$ effects dominate over the MSSM, but the number of signal events barely reaches one in some cases. This means that for a clear extraction of the $U(1)^{\prime}$ effects, higher integrated luminosities (than $100 \mathrm{fb})^{-1}$ ) are needed.
One has to keep in mind that these conclusions are based on the generator-level analysis. The next step of such an analysis would be to have a more realistic picture of what is experimentally feasible by implementing a full detector analysis. This is currently being implemented in the CMSSW analysis system of the CMS experiment [25].

If the analysis in this work, together with the close-up provided by the simulation study in progress, has taught us anything, it is that the search for the extra gauge interactions, in the supersymmetric framework, must proceed through not only the forces mediated by gauge bosons (which have been under study both phenomenologically and experimentally [7]) but also the by the forces mediated by the gauge fermions. Our analysis has been limited to the $U(1)^{\prime}$ model; however, the discussions in Sec. III, together with the various distributions simulated, should provide enough guidance for the expectations about more general models, such as the left-right symmetric models or the $3-$ $3-1$ models.

\section{ACKNOWLEDGMENTS}

The work of M. F. and I. T. was supported in part by the NSERC of Canada under Grant No. SAP01105354. The work of D. D. was supported in part by the Alexander von Humboldt-Stiftung through Friedrich Wilhelm BesselForschungspreis, the Turkish Academy of Sciences via a GEBIP grant, and the Turkish Atomic Energy Agency (TAEK) via the CERN-CMS Research Grant. We thank Lisa Everett and Paul Langacker for reading the manuscript and making important comments, Antonio Masiero for highly illuminating discussions, Orhan Çakır for helpful communications about the Pythia package, and Neil Christensen for fruitful discussions about the CalcHEP package. We thank Shabaan Khalil and Hiroshi Okada for discussions about the low-scale $U(1)_{B-L}$. We also thank the participants of the DARK 2008 Conference, the LPC Dijet Group Meetings, the ATLAS Canada/Carleton LHC Theory Workshop, and the TAEK Annual Meeting for comments and suggestions on different aspects of this work.

\section{APPENDIX A: THE LAGRANGIAN}

In this appendix we provide the Lagrangian of the $U(1)^{\prime}$ model and compare its certain features with those of the MSSM Lagrangian. The particle spectrum of the model with the generic $U(1)^{\prime}$ hypercharge assignments is given in Table VI. The total Lagrangian involves kinetic terms as well as various interaction terms among the fields. We discuss below the distinct pieces separately.

The kinetic terms of the Lagrangian are given by

$$
\begin{aligned}
\mathcal{L}_{U(1)^{\prime}}^{\text {Kinetic }}= & \mathcal{L}_{\mathrm{MSSM}}^{\text {Kinetic }}-\frac{1}{4} Z^{\prime \mu \nu} Z_{\mu \nu}^{\prime}+\left(\mathcal{D}_{\mu} S\right)^{\dagger}\left(\mathcal{D}^{\mu} S\right) \\
& +\tilde{Z}^{\prime \dagger} i \sigma^{\mu} \partial_{\mu} \tilde{Z}^{\prime}+\tilde{S}^{\dagger} i \sigma^{\mu} \mathcal{D}_{\mu} \tilde{S}
\end{aligned}
$$


TABLE VI. The field content of the $U(1)^{\prime}$ model based on $G_{\mathrm{SM}} \otimes U(1)^{\prime}$ gauge invariance. The $U(1)^{\prime}$ charges listed here are the ones in (3) for which the kinetic mixing vanishes.

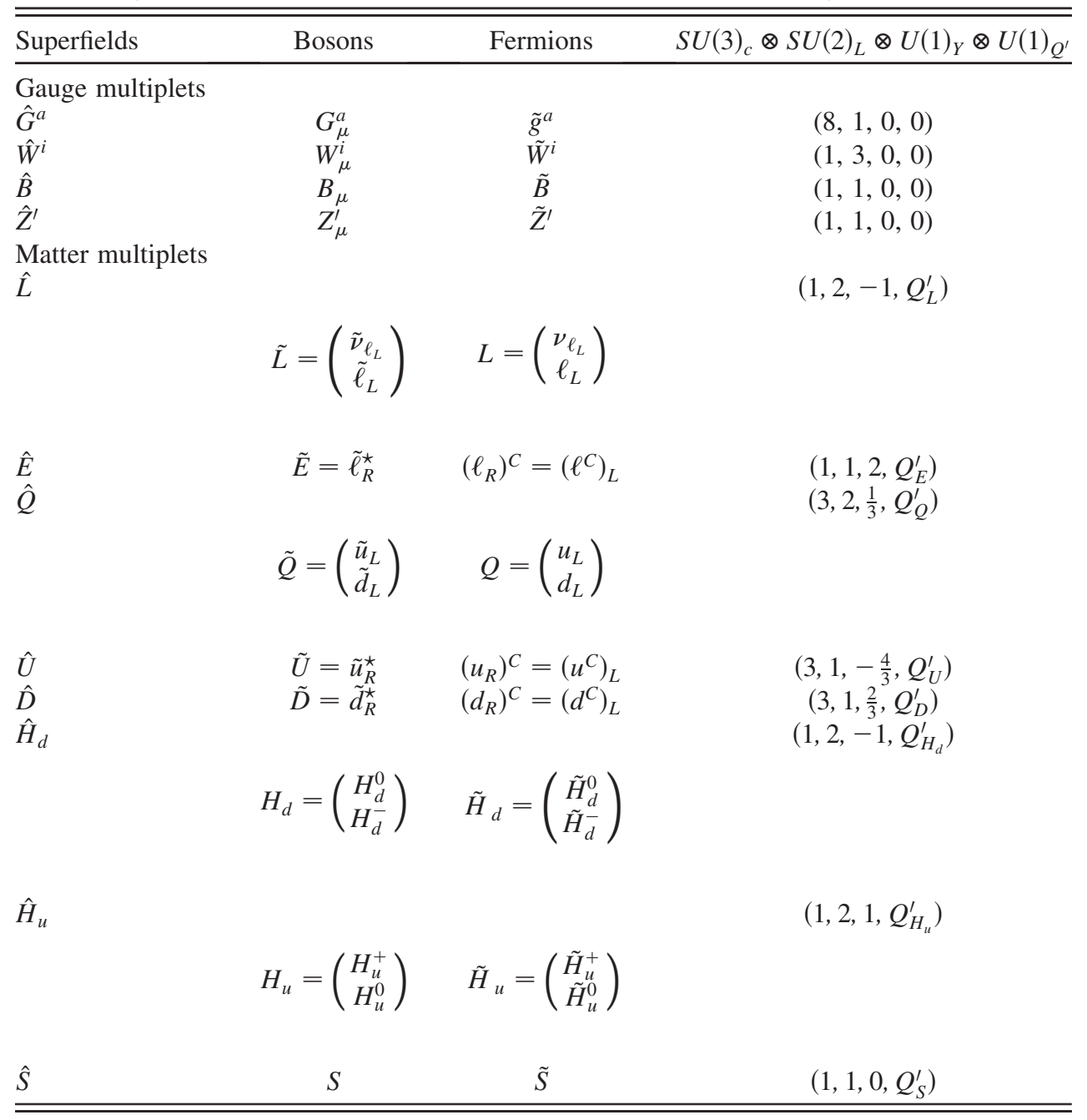

and the interactions of the gauge fields with the rest (fermions, sfermions, gauginos, Higgs and Higgsino fields) are contained in the piece

$$
\mathcal{L}_{U(1)^{\prime}}^{\text {gauge }}=\mathcal{L}_{\mathrm{MSSM}}^{\text {gauge }}\left(g_{Y} \frac{Y_{X}}{2} B_{\mu} \rightarrow g_{Y} \frac{Y_{X}}{2} B_{\mu}+g_{Y^{\prime}} Q_{X}^{\prime} Z_{\mu}^{\prime}\right),
$$

where $X$ runs over the fields charged under $U(1)_{O}^{\prime}$. In (A1), $Z^{\prime \mu \nu}$ is the field strength tensor of $Z_{\mu}^{\prime}$, and $\mathcal{D}_{\mu} S=\left(\partial_{\mu}+\right.$ $\left.i g_{Y^{\prime}} Q_{S}^{\prime} Z_{\mu}^{\prime}\right) S$.

Given the superpotential in (1), part of the $U(1)^{\prime}$ Lagrangian spanned by the $F$-terms is given by

$$
\begin{aligned}
\mathcal{L}_{U(1)^{\prime}}^{F-\text { term }}= & -\sum_{i}\left|\frac{\partial W}{\partial \phi_{i}}\right|^{2}=\mathcal{L}_{\operatorname{MSSM}}^{F-\operatorname{term}}\left(\mu \rightarrow h_{s} S\right) \\
& -h_{s}^{2}\left|H_{u} \cdot H_{d}\right|^{2},
\end{aligned}
$$

where $\phi_{i}$ is the scalar component of the $i$-the chiral superfield in the superpotential.
The $D$-term contributions to the Lagrangian are given by

$$
\begin{aligned}
\mathcal{L}_{U(1)^{\prime}}^{D \text {-term }}= & -\frac{1}{2} \sum_{a} D^{a} D^{a} \\
= & \mathcal{L}_{\mathrm{MSSM}}^{D \text {-term }}-\frac{g_{Y^{\prime}}^{2}}{8}\left(Q_{Q}^{\prime} \tilde{Q}^{\dagger} \tilde{Q}+Q_{U}^{\prime} \tilde{u}_{R}^{T} \tilde{u}_{R}^{\star}\right. \\
& +Q_{D}^{\prime} \tilde{d}_{R}^{T} \tilde{d}_{R}^{\star}+Q_{L}^{\prime} \tilde{L}^{\dagger} \tilde{L}+Q_{E}^{\prime} \tilde{E}^{T} \tilde{E}^{\star} \\
& \left.+Q_{H_{d}}^{\prime} H_{d}^{\dagger} H_{d}+Q_{H_{u}}^{\prime} H_{u}^{\dagger} H_{u}+Q_{S}^{\prime} S^{\dagger} S\right)^{2} .
\end{aligned}
$$

The soft-breaking sector of the $U(1)^{\prime}$ Lagrangian is given by

$$
\begin{aligned}
\mathcal{L}_{U(1)^{\prime}}^{\text {Soft }}= & \mathcal{L}_{\mathrm{MSSM}}^{\text {Soft }}(\mu \rightarrow 0)-m_{S^{2}}^{2} S^{\dagger} S-\left[h_{s} A_{s} S H_{u} \cdot H_{d}+\text { H.c. }\right] \\
& +\frac{1}{2}\left(M_{\tilde{Z}^{\prime}} \tilde{Z}^{\prime} \tilde{Z}^{\prime}+M_{\tilde{Y} \tilde{Z}^{\prime}} \tilde{Y} \tilde{Z}^{\prime}+\text { H.c. }\right)
\end{aligned}
$$

where $M_{\tilde{Y} \tilde{Z}^{\prime}}$ and $M_{\tilde{Z}^{\prime}}$ are defined below the neutralino mass matrix in (5), and $A_{s}$ is the extra trilinear soft coupling. 
Finally, the part of the Lagrangian consisting of the fermion-sfermion-ino as well as the Higgs-HiggsinoHiggsino interactions is given by

$$
\begin{aligned}
\mathcal{L}_{U(1)^{\prime}}^{\mathrm{ino}-f-\phi}= & \mathcal{L}_{\mathrm{MSSM}}^{\mathrm{ino}-f-\phi}(\mu \rightarrow 0)+i \sqrt{2} g_{Y^{\prime}}\left[Q_{Q}^{\prime} Q^{\dagger} \tilde{Z}^{\prime} \tilde{Q}\right. \\
& +Q_{U}^{\prime} u_{R}^{\dagger} \tilde{Z}^{\prime} \tilde{u}_{R}+Q_{D}^{\prime} d_{R}^{\dagger} \tilde{Z}^{\prime} \tilde{d}_{R}+Q_{L}^{\prime} L^{\dagger} \tilde{Z}^{\prime} \tilde{L} \\
& +Q_{E}^{\prime} \ell_{R}^{\dagger} \tilde{Z}^{\prime} \tilde{\ell}_{R}+Q_{H_{d}}^{\prime} \tilde{H}_{d}^{\dagger} \tilde{Z}^{\prime} H_{d}+Q_{H_{u}}^{\prime} \tilde{H}_{u}^{\dagger} \tilde{Z}^{\prime} H_{u} \\
& \left.+Q_{S}^{\prime} \tilde{S}^{\dagger} \tilde{Z}^{\prime} S+\text { H.c. }\right]+\left[h_{s} S \tilde{H}_{u} \cdot \tilde{H}_{d}\right. \\
& \left.+h_{s} \tilde{S} H_{u} \cdot \tilde{H}_{d}+h_{s} \tilde{S} \tilde{H}_{u} \cdot H_{d}+\text { H.c. }\right] . \quad(\mathrm{A} 6)
\end{aligned}
$$

All parts of the $G_{\mathrm{SM}} \otimes U(1)^{\prime}$ Lagrangian listed above are in the current basis. Eventually, the fields must be transformed into the physical basis wherein each field obtains a definite mass. The neutral gauginos and Higgsinos form the neutralino sector whose physical states are expressed as (4) after diagonalizing the mass matrix (5). The chargino sector is essentially the same as in the MSSM with the obvious replacement $\mu \rightarrow h_{s} v_{s} / \sqrt{2}$. The Higgs sector has been analyzed in detail at one-loop level in [12].

The kinetic mixing in (2) can be eliminated via the transformation

$$
\left(\begin{array}{c}
\hat{W}_{Y} \\
\hat{W}_{Y^{\prime}}
\end{array}\right)=\left(\begin{array}{cc}
1 & -\tan \chi \\
0 & 1 / \cos \chi
\end{array}\right)\left(\begin{array}{c}
\hat{W}_{B} \\
\hat{W}_{Z^{\prime}}
\end{array}\right)
$$

where the kinetic eigenstates $\hat{W}_{B}$ and $\hat{W}_{Z^{\prime}}$ couple to a matter field $f$ (with hypercharge $Y_{f}$ and the $U(1)_{Y^{\prime}}$ charge $Y_{f}^{\prime}$ ) with strengths $g_{Y} Y_{f}$ and $g_{Y^{\prime}} Q_{f}^{\prime}$, respectively. Consequently, the boson sector extends the MSSM gauge boson sector by the $Z^{\prime}$ gauge boson of the $U(1)_{Q^{\prime}}$ group, and the Higgs sector by a new singlet field.

In the gauge boson sector, spontaneous breakdown of the product group $S U(2)_{L} \otimes U(1)_{Y} \otimes U(1)_{Q^{\prime}}$ via the Higgs VEVs,

$$
\left\langle H_{u}\right\rangle=\frac{1}{\sqrt{2}}\left(\begin{array}{c}
0 \\
v_{u}
\end{array}\right), \quad\left\langle H_{d}\right\rangle=\frac{1}{\sqrt{2}}\left(\begin{array}{c}
v_{d} \\
0
\end{array}\right), \quad\langle S\rangle=\frac{v_{s}}{\sqrt{2}},
$$

generates one massless state (photon) and a massive state ( $Z$ boson) via two orthonormal combinations of $W_{\mu}^{3}$ and $B_{\mu}$ gauge bosons. The $W_{\mu}^{1}$ and $W_{\mu}^{2}$ linearly combine to give $W_{\mu}^{ \pm}$, as the only charged vector bosons in the model. In contrast to the MSSM, the $Z$ boson is not a physical state by itself since it mixes with the $Z^{\prime}$ boson. This mass mixing arises from the fact that the Higgs doublets $H_{u, d}$ are charged under each factor of $S U(2)_{L} \otimes U(1)_{Y} \otimes U(1)_{Q^{\prime}}$, and the associated mass-squared matrix is given by $[3,7]$

$$
M_{Z-Z^{\prime}}^{2}=\left(\begin{array}{cc}
M_{Z}^{2} & \Delta^{2} \\
\Delta^{2} & M_{Z^{\prime}}^{2}
\end{array}\right)
$$

in the $\left(Z_{\mu}, Z_{\mu}^{\prime}\right)$ basis. Its entries are

$$
\begin{aligned}
M_{Z}^{2} & =\frac{1}{4} G_{Z}^{2}\left(v_{u}^{2}+v_{d}^{2}\right), \\
M_{Z^{\prime}}^{2} & =g_{Y^{\prime}}^{2}\left(Q_{H_{u}}^{2} v_{u}^{2}+Q_{H_{d}}^{\prime 2} v_{d}^{2}+Q_{S}^{\prime 2} v_{s}^{2}\right), \\
\Delta^{2} & =\frac{1}{2} G_{Z} g_{Y^{\prime}}\left(Q_{H_{u}}^{\prime} v_{u}^{2}-Q_{H_{d}}^{\prime} v_{d}^{2}\right),
\end{aligned}
$$

where $G_{Z}^{2}=g_{2}^{2}+g_{Y}^{2}$. The physical neutral vector bosons, $Z_{1,2}$, are obtained by diagonalizing $M_{Z-Z^{\prime}}^{2}$ :

$$
\left(\begin{array}{l}
Z_{1} \\
Z_{2}
\end{array}\right)=\left(\begin{array}{cc}
\cos \theta_{Z-Z^{\prime}} & \sin \theta_{Z-Z^{\prime}} \\
-\sin \theta_{Z-Z^{\prime}} & \cos \theta_{Z-Z^{\prime}}
\end{array}\right)\left(\begin{array}{c}
Z \\
Z^{\prime}
\end{array}\right),
$$

where

$$
\theta_{Z-Z^{\prime}}=-\frac{1}{2} \arctan \left(\frac{2 \Delta^{2}}{M_{Z^{\prime}}^{2}-M_{Z}^{2}}\right)
$$

is their mass mixing angle, and

$$
M_{Z_{1(2)}}^{2}=\frac{1}{2}\left[M_{Z^{\prime}}^{2}+M_{Z}^{2}-(+) \sqrt{\left(M_{Z^{\prime}}^{2}-M_{Z}^{2}\right)^{2}+4 \Delta^{4}}\right]
$$

are their squared masses. The collider searches at LEP and Tevatron plus various indirect observations require $Z-Z^{\prime}$ mixing angle $\theta_{Z-Z^{\prime}}$ to be at most a few $10^{-3}$ with an unavoidable model dependence coming from the $Z^{\prime}$ couplings [7-10,26,27]. This bound requires either $M_{Z_{2}}$ to be large enough (well in the $\mathrm{TeV}$ range) or $\Delta^{2}$ to be sufficiently suppressed by the vacuum configuration, that is, $\tan ^{2} \beta \equiv v_{u}^{2} / v_{d}^{2} \sim Q_{H_{d}}^{\prime} / Q_{H_{u}}^{\prime}$. Which of these options is realized depends on the $U(1)^{\prime}$ charge assignments and the soft-breaking masses in the Higgs sector (see [28] for a variant reducing the $Z-Z^{\prime}$ mixing).

In the Higgs sector, the $U(1)^{\prime}$ model consists of an extra $C P$-even Higgs boson, $H^{\prime}$ with a mass $m_{H^{\prime}} \sim M_{Z^{\prime}}$ stemming from the extra chiral field $\hat{S}$, the scalar component of which is responsible for generating the $\mu$ parameter. There is no new $C P$-odd scalar since the imaginary parts of $H_{u}^{0}$, $H_{d}^{0}$ and $S$ combine to give masses to the $Z$ and $Z^{\prime}$ bosons, leaving behind a single $C P$-odd Higgs boson $A^{0}$ as in the MSSM. Consequently, in terms of the Higgs boson spectrum, the $U(1)^{\prime}$ model differs from the MSSM only in having an extra $C P$-even Higgs boson, $H^{\prime}$. This feature, however, is not necessarily the most important one given that the mass spectra of the Higgs bosons differ significantly in the two models. Indeed, the lightest Higgs boson $h$ in the $U(1)^{\prime}$ model weighs well above $M_{Z}$ already at tree level [3], and thus, large radiative corrections (and hence large top-stop mass splitting) are not warranted to satisfy the LEP lower bound on $m_{h}$ [12-14]. This property can prove useful in moderating the little hierarchy problem (especially when a set of the MSSM singlet chiral fields are included to form a secluded sector [19]). 


\section{APPENDIX B: THE SCALAR FERMIONS}

Given rather tight flavor-changing neutral-currents (FCNC) bounds, we neglect all the intergenerational mixings, and consider only intragenerational left-right mixings, though these turn out to be totally negligible for the sfermions in the first and second generations. The $2 \times 2$ scalar fermion mixing matrix can be written as

$$
\mathcal{M}_{\tilde{f}^{a}}^{2}=\left(\begin{array}{cc}
\mathcal{M}_{\tilde{f}_{L L}^{a}}^{2} & \mathcal{M}_{\tilde{f}_{L R}^{a, b}}^{2} \\
\mathcal{M}_{\tilde{f}_{L R}^{a, b}}^{2+} & \mathcal{M}_{\tilde{f}_{R R}^{a}}^{2}
\end{array}\right), \quad a \neq b=u, d,
$$

where

$$
\begin{gathered}
\mathcal{M}_{\tilde{f}_{L L}^{a}}^{2}=m_{\tilde{f}_{L}}^{2}+h_{f^{a}}^{2} v_{a}^{2}+\frac{1}{2}\left(g_{Y}^{2} \frac{Y_{f_{L}^{a}}}{2}-g_{2}^{2} T_{3 L}\right)\left(v_{u}^{2}-v_{d}^{2}\right) \\
+g_{Y^{\prime}}^{2} Q_{f_{L}^{a}}^{\prime}\left(Q_{H_{u}} v_{u}^{2}+Q_{H_{d}} v_{d}^{2}+Q_{s} v_{s}^{2}\right), \\
\mathcal{M}_{\tilde{f}_{L R}^{a, b}}^{2}=h_{f^{a}}\left(A_{f^{a}} v_{a}-h_{s} v_{s} v_{b}\right), \\
\mathcal{M}_{\tilde{f}_{R R}^{a}}^{2}=m_{\tilde{f}_{R}}^{2}+h_{f^{a}}^{2} v_{a}^{2}+\frac{1}{2}\left(g_{Y}^{2} \frac{Y_{f_{L}^{a}}}{2}\right)\left(v_{u}^{2}-v_{d}^{2}\right) \\
+g_{Y^{\prime}}^{2} Q_{f_{R}^{a}}^{\prime}\left(Q_{H_{u}} v_{u}^{2}+Q_{H_{d}} v_{d}^{2}+Q_{s} v_{s}^{2}\right) .
\end{gathered}
$$

Here $m_{\tilde{f}_{L, R}^{2}}$ are the soft mass-squared of the sfermions, $v_{u, d, s}$ are the VEVs of the Higgs fields, $Y_{f^{a}}\left(T_{3 L}\right)$ is the $U(1)_{Y}\left(S U(2)_{L}\right)$ quantum number, $Q_{f^{a}}^{\prime}$ is the $U(1)^{\prime}$ charge and $A_{f^{a}}$ are the trilinear couplings. The mixing matrix can be diagonalized, in general, by a unitary matrix $\Gamma^{f}$ such that $\Gamma^{f^{a} \dagger} \cdot \mathcal{M}_{\tilde{f}^{a}}^{2} \cdot \Gamma^{f^{a}} \equiv \operatorname{Diag}\left(M_{\tilde{f}_{1}^{a}}^{2}, M_{\tilde{f}_{2}^{a}}^{2}\right){ }^{1}$ The rotation matrix $\Gamma^{f^{a}}$ can be written for quarks and charged leptons in the $2 \times 2\left\{\tilde{f}_{L}^{a}, \tilde{f}_{R}^{a}\right\}$ basis as

$$
\Gamma^{f^{a}}=\left(\begin{array}{cc}
\cos \theta_{\tilde{f}^{a}} & -\sin \theta_{\tilde{f}^{a}} \\
\sin \theta_{\tilde{f}^{a}} & \cos \theta_{\tilde{f}^{a}}
\end{array}\right),
$$

where $\quad \theta_{\tilde{f}^{a}}=\frac{1}{2} \arctan 2\left(-2 \mathcal{M}_{\tilde{f}_{L R}^{a}}^{2}, \mathcal{M}_{\tilde{f}_{R R}^{a}}^{2}-\mathcal{M}_{\tilde{f}_{L L}^{a}}^{2}\right) \quad$ and $\arctan 2(y, x)$ is defined as

$$
\arctan 2(y, x)= \begin{cases}\phi \operatorname{sign}(y), & x>0 \\ \frac{\pi}{2} \operatorname{sign}(y), & x=0 \\ (\pi-\phi) \operatorname{sign}(y), & x<0\end{cases}
$$

with $y$ being nonzero, and $\phi$ taken in the first quadrant such that $\tan \phi=|y / x|$.

For the sfermions in the first and second generations, the left-right mixings are exceedingly small as they are proportional to the corresponding fermion mass. Therefore, the sfermion mass matrix (B2) is automatically diagonal. However, one has to remember that the sfermion masses, for fixed values of $m_{\tilde{f}_{L, R}^{2}}$, are different in the MSSM and the

\footnotetext{
${ }^{1}$ We note that unlike mixings in other sectors, $\Gamma^{f^{a}}$ is defined differently, that is, $\left(\tilde{f}_{L, R}^{a}\right)_{i}=\Gamma_{i j}^{f a} \tilde{f}_{j}^{a}$, where $\tilde{f}_{j}^{a}$ represent the masseigenstates.
}

$U(1)^{\prime}$ models due to the additional $D$-term contribution in the latter. This is the reason for having different squark masses in the plots of branching ratios in Figs. 1-3 for the parameter set in (32).

\section{APPENDIX C: THE FERMION-SFERMION- NEUTRALINO COUPLINGS}

In this appendix we list the neutralino couplings relevant for the production and decays of the squarks and sleptons. ${ }^{2}$ The six physical neutralinos

$$
\tilde{\chi}_{j}^{0}=\sum_{a} N_{j a}^{0} \tilde{G}_{a}
$$

couple to the fermions and the scalar fermions. The neutralino-quark-scalar quark couplings read as

$$
\begin{gathered}
\bar{u}^{k} \tilde{\chi}_{j}^{0} \tilde{u}_{\alpha}^{k}-i\left[\sqrt { 2 } \Gamma _ { \alpha 1 } ^ { u _ { k } } \left(\frac{e}{6 \cos \theta_{W}} N_{j 1}^{0}+\frac{e}{2 \sin \theta_{W}} N_{j 2}^{0}\right.\right. \\
\left.\left.+Q_{Q}^{\prime} g_{Y}^{\prime} N_{j 6}^{0}\right)+Y_{u_{k}} N_{j 4}^{0} \Gamma_{\alpha 2}^{u_{k}}\right] P_{R} \\
+i\left[\sqrt{2} \Gamma_{\alpha 2}^{u_{k}}\left(\frac{2 e}{3 \cos \theta_{W}} N_{j 1}^{0}-Q_{Q}^{\prime} g_{Y}^{\prime} N_{j 6}^{0}\right)\right. \\
\left.-Y_{u_{k}} N_{j 4}^{0} \Gamma_{\alpha 1}^{u_{k}}\right] P_{L}, \\
\bar{d}^{k} \tilde{\chi}_{j}^{0} \tilde{d}_{\alpha}^{k}-i\left[\sqrt { 2 } \Gamma _ { \alpha 1 } ^ { d _ { k } } \left(\frac{e}{6 \cos \theta_{W}} N_{j 1}^{0}-\frac{e}{2 \sin \theta_{W}} N_{j 2}^{0}\right.\right. \\
\left.\left.+Q_{Q}^{\prime} g_{Y}^{\prime} N_{j 6}^{0}\right)-Y_{d_{k}} N_{j 4}^{0} \Gamma_{\alpha 2}^{d_{k}}\right] P_{R} \\
+i\left[\sqrt{2} \Gamma_{\alpha 2}^{d_{k}}\left(\frac{-e}{3 \cos \theta_{W}} N_{j 1}^{0}-Q_{Q}^{\prime} g_{Y}^{\prime} N_{j 6}^{0}\right)\right. \\
\left.+Y_{d_{k}} N_{j 4}^{0} \Gamma_{\alpha 1}^{d_{k}}\right] P_{L},
\end{gathered}
$$

where $\alpha=1,2$ designates the squark mass-eigenstates, $k$ is the generation label, $\Gamma_{\alpha i}^{q_{k}}$ are the squark mixing matrices, assumed diagonal for the first two generations so that $\Gamma_{i j}^{u_{k}\left(d_{k}\right)}=\delta_{i j}$ for $k=1,2$, and finally, $Y_{q_{k}}$ are the quark Yukawa couplings. by

The neutralino-lepton-scalar lepton couplings are given

$$
\begin{gathered}
\bar{l}^{k} \tilde{\chi}_{j}^{0} \tilde{l}_{\alpha}^{k} i\left[\sqrt{2} \Gamma_{\alpha 1}^{l_{k}}\left(\frac{e}{\cos \theta_{W}} N_{j 1}^{0}+\frac{e}{\sin \theta_{W}} N_{j 2}^{0}-Q_{L}^{\prime} g_{Y}^{\prime} N_{j 6}^{0}\right)\right. \\
\left.+Y_{l_{k}} N_{j 4}^{0} \Gamma_{\alpha 2}^{l_{k}}\right] P_{R}-i\left[\sqrt { 2 } \Gamma _ { \alpha 2 } ^ { l _ { k } } \left(\frac{e}{\cos \theta_{W}} N_{j 1}^{0}\right.\right. \\
\left.\left.+Q_{E}^{\prime} g_{Y}^{\prime} N_{j 6}^{0}\right)-Y_{l_{k}} N_{j 4}^{0} \Gamma_{\alpha 1}^{l_{k}}\right] P_{L}
\end{gathered}
$$

\footnotetext{
${ }^{2}$ The couplings of the $Z_{1,2}$ bosons to the fermions and neutralinos as well as the couplings of the neutralinos to the fermions and sfermions are given in Sec. IV of [5], which were used for cross-checking.
} 




diagr. 1

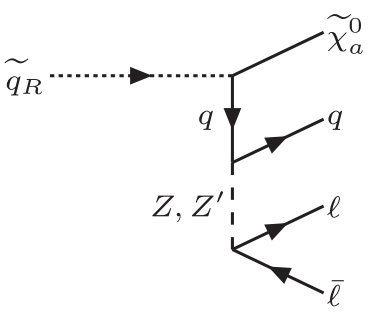

diagr.5

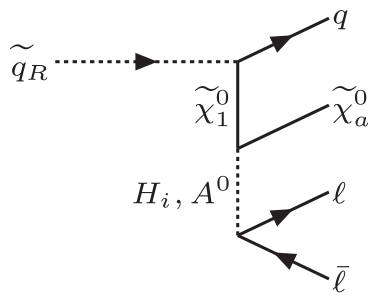

$\operatorname{diagr.2}$

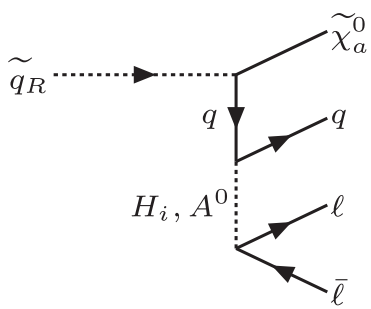

diagr. 6

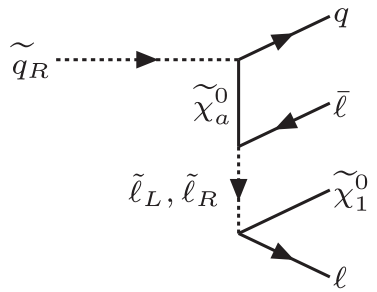

diagr.3

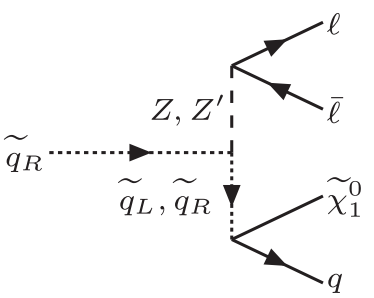

diagr.7



$\operatorname{diagr.4}$

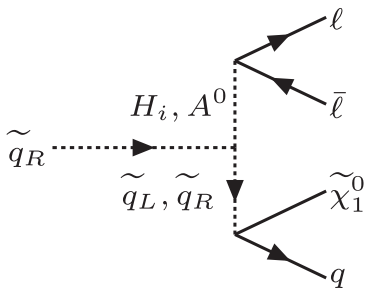

$\operatorname{diagr.8}$

FIG. 22. The Feynman diagrams governing the $\tilde{q}_{R} \rightarrow q \ell^{-} \ell^{+}+\tilde{\chi}_{1}^{0}$ decay in the $U(1)^{\prime}$ model. Here the index a runs from 1 to 6 and the index i from 1 to 3 .

$$
\bar{\nu}^{k} \tilde{\chi}_{j}^{0} \tilde{\nu}^{k} i\left[\sqrt{2}\left(\frac{e}{\cos \theta_{W}} N_{j 1}^{0}-\frac{e}{\sin \theta_{W}} N_{j 2}^{0}-Q_{L}^{\prime} g_{Y}^{\prime} N_{j 6}^{0}\right)\right] P_{R},
$$

where $\Gamma_{i j}^{l_{k}}$, the slepton mixing matrix, is diagonal $\Gamma_{i j}^{l_{k}}=\delta_{i j}$ for $k=1,2$ (corresponding to the electron and the muon).

The charginos couple to the fermions and scalar fermions in the same manner as in the MSSM.

\section{APPENDIX D: AN EXAMPLE OF FEYNMAN DIAGRAMS}

In this appendix we include, for illustration, the Feynman diagrams contributing to the processes which have been analyzed in the text. We have implemented the model Lagrangian and all the information contained in the previous appendices into a $\mathrm{Ca}$ CHEP code for simulation study. We illustrate the computer code in Fig. 22 by picking up $\tilde{q}_{R}$ decays as an example. We note that even though the diagrams in Fig. 22 are presented as 4-body modes, we use the narrow-width approximation, and the squarks are assumed to have a 2-body decay at first, and then, the neutralino exhibits a 3-body decay to make up a 4-body final state. In this respect, the diagrams 5 and 6 in Fig. 22 do not contribute due to cascade decays. For the same reason, the diagrams 7 and 8 do not contribute either.
[1] S. Cecotti, J. P. Derendinger, S. Ferrara, L. Girardello, and M. Roncadelli, Phys. Lett. B 156, 318 (1985); J. D. Breit, B. A. Ovrut, and G. C. Segre, Phys. Lett. B 158, 33 (1985); S. M. Barr, Phys. Rev. Lett. 55, 2778 (1985); F. del Aguila, G. A. Blair, M. Daniel, and G. G. Ross, Nucl. Phys. B272, 413 (1986); J. L. Hewett and T. G. Rizzo, Phys. Rep. 183, 193 (1989); M. Cvetic and P. Langacker, Phys. Rev. D 54, 3570 (1996); G. Cleaver, M. Cvetic, J. R. Espinosa, L. L. Everett, and P. Langacker, Phys. Rev. D 57, 2701 (1998); Nucl. Phys. B525, 3 (1998); D. M. Ghilencea, L.E. Ibanez, N. Irges, and F. Quevedo, J. High Energy Phys. 08 (2002) 016; S. F. King, S. Moretti, and R. Nevzorov, Phys. Rev. D 73, 035009 (2006).

[2] J. E. Kim and H. P. Nilles, Phys. Lett. B 138, 150 (1984); D. Suematsu and Y. Yamagishi, Int. J. Mod. Phys. A 10, 4521 (1995); M. Cvetic and P. Langacker, Mod. Phys.
Lett. A 11, 1247 (1996); V. Jain and R. Shrock, arXiv:hepph/9507238; D. A. Demir, Phys. Rev. D 59, 015002 (1998); H. S. Lee, K. T. Matchev, and T. T. Wang, Phys. Rev. D 77, 015016 (2008); D. A. Demir, L. L. Everett, and P. Langacker, Phys. Rev. Lett. 100, 091804 (2008).

[3] M. Cvetic, D. A. Demir, J. R. Espinosa, L. L. Everett, and P. Langacker, Phys. Rev. D 56, 2861 (1997); 58, 119905 (E) (1998); P. Langacker and J. Wang, Phys. Rev. D 58, 115010 (1998).

[4] K. S. Babu, C. F. Kolda, and J. March-Russell, Phys. Rev. D 57, 6788 (1998); T. G. Rizzo, Phys. Rev. D 59, 015020 (1998).

[5] S. Y. Choi, H. E. Haber, J. Kalinowski, and P. M. Zerwas, Nucl. Phys. B778, 85 (2007).

[6] V. Barger, P. Langacker, and H. S. Lee, Phys. Lett. B 630, 85 (2005); V. Barger, P. Langacker, and G. Shaughnessy, 
Phys. Lett. B 644, 361 (2007); H. S. Lee, K. T. Matchev, and S. Nasri, Phys. Rev. D 76, 041302(R) (2007).

[7] P. Langacker, arXiv:0801.1345.

[8] P. Langacker, R. W. Robinett, and J. L. Rosner, Phys. Rev. D 30, 1470 (1984); F. del Aguila, M. Quiros, and F. Zwirner, Nucl. Phys. B284, 530 (1987); Nucl. Phys. B287, 419 (1987); M. Cvetic and B. W. Lynn, Phys. Rev. D 35, 51 (1987); F. del Aguila, M. Cvetic, and P. Langacker, Phys. Rev. D 48, R969 (1993); 52, 37 (1995); F. del Aguila and M. Cvetic, Phys. Rev. D 50, 3158 (1994); A. Leike, Phys. Lett. B 402, 374 (1997); Phys. Rep. 317, 143 (1999); T. Appelquist, B. A. Dobrescu, and A. R. Hopper, Phys. Rev. D 68, 035012 (2003); M. Dittmar, A. S. Nicollerat, and A. Djouadi, Phys. Lett. B 583, 111 (2004); A. Freitas, Phys. Rev. D 70, 015008 (2004); M. Carena, A. Daleo, B. A. Dobrescu, and T. M. P. Tait, Phys. Rev. D 70, 093009 (2004); F. Petriello and S. Quackenbush, Phys. Rev. D 77, 115004 (2008); C. Coriano, A. E. Faraggi and M. Guzzi, Phys. Rev. D 78, 015012 (2008).

[9] J. Kang and P. Langacker, Phys. Rev. D 71, 035014 (2005).

[10] For LEP bounds see: LEP Collaboration, arXiv:hep-ex/ 0312023; For most recent bounds from Tevatron see: CDF Report No. 9160.

[11] See the URL: http://www-cdf.fnal.gov/physics/exotic/r2a/ 20080306.dielectron-duke/pub25/cdfnote9160-pub.pdf.

[12] D. A. Demir and L. L. Everett, Phys. Rev. D 69, 015008 (2004).

[13] E. Keith and E. Ma, Phys. Rev. D 56, 7155 (1997); D. A. Demir and N. K. Pak, Phys. Rev. D 57, 6609 (1998); Phys. Lett. B 439, 309 (1998); H. Amini, New J. Phys. 5, 49 (2003); S. W. Ham, E. J. Yoo, and S. K. Oh, Phys. Rev. D 76, 015004 (2007); W. Emam and S. Khalil, Eur. Phys. J. C 52, 625 (2007); S. W. Ham, T. Hur, P. Ko, and S. K. Oh, J. Phys. G 35, 095007 (2008).

[14] T. Han, P. Langacker, and B. McElrath, Phys. Rev. D 70, 115006 (2004); V. Barger, P. Langacker, H. S. Lee, and G. Shaughnessy, Phys. Rev. D 73, 115010 (2006); V. Barger, P. Langacker, and G. Shaughnessy, Phys. Rev. D 75, 055013 (2007); V. Barger, P. Langacker, M. McCaskey, M. J. Ramsey-Musolf, and G. Shaughnessy, Phys. Rev. D
77, 035005 (2008).

[15] G. J. Gounaris, J. Layssac, P. I. Porfyriadis, and F. M. Renard, Phys. Rev. D 70, 033011 (2004); T. Wohrmann and H. Fraas, Phys. Rev. D 52, 78 (1995); H. Baer, C. H. Chen, F. Paige, and X. Tata, Phys. Rev. D 50, 4508 (1994).

[16] S. Abdullin et al. (CMS Collaboration), J. Phys. G 28, 469 (2002); A. J. Barr, Report No. CERN-THESIS-2004-002, 2002.

[17] S. Dawson, E. Eichten, and C. Quigg, Phys. Rev. D 31, 1581 (1985); W. Beenakker, R. Hopker, M. Spira, and P. M. Zerwas, Nucl. Phys. B492, 51 (1997); A. T. Alan, K. Cankocak, and D. A. Demir, Phys. Rev. D 75, 095002 (2007); 76, 119903 (2007); S. Bornhauser, M. Drees, H. K. Dreiner, and J.S. Kim, Phys. Rev. D 76, 095020 (2007).

[18] F.E. Paige, arXiv:hep-ph/9801254; D. R. Tovey, Phys. Lett. B 498, 1 (2001); M. Tytgat, arXiv:0710.1013.

[19] D. A. Demir, G. L. Kane, and T. T. Wang, Phys. Rev. D 72, 015012 (2005).

[20] K. S. Babu, C. F. Kolda, and J. March-Russell, Phys. Rev. D 54, 4635 (1996).

[21] See the URL: http://theory.sinp.msu.ru/ pukhov/calchep. html; A. Pukhov, arXiv:hep-ph/0412191.

[22] A. Semenov, Comput. Phys. Commun. 180, 431 (2009); A. Semenov, Comput. Phys. Commun. 115, 124 (1998).

[23] T. Sjostrand, S. Mrenna, and P. Skands, J. High Energy Phys. 05 (2006) 026.

[24] D. A. Demir, M. Frank, K. Huitu, S. K. Rai, and I. Turan, Phys. Rev. D 78, 035013 (2008).

[25] K. Cankocak, D. Demir, M. Frank, and I. Turan (LPC Dijet Group in the CMS Collaboration), Dijet Studies in SUSY with Extra $U(1)$.

[26] U. Amaldi et al., Phys. Rev. D 36, 1385 (1987); P. Langacker, M. x. Luo, and A. K. Mann, Rev. Mod. Phys. 64, 87 (1992); J. Erler and P. Langacker, Phys. Rev. Lett. 84, 212 (2000); Phys. Lett. B 592, 1 (2004); Phys. Lett. B 456, 68 (1999).

[27] A. Fiandrino and P. Taxil, Phys. Rev. D 44, 3490 (1991); P. Taxil, E. Tugcu, and J. M. Virey, Eur. Phys. J. C 24, 149 (2002).

[28] J. Erler, P. Langacker, and T. Li, Phys. Rev. D 66, 015002 (2002). 